\title{
Zinc Amide-Catalyzed Regioselective Allenylation and Propargylation of Ketones with Allenyl Boronate
}

\author{
Yasuhiro Yamashita, Yi Cui, Peizhong Xie, and Sh $\bar{u}$ Kobayashi* \\ Department of Chemistry, School of Science, The University of Tokyo, Hongo, \\ Bunkyo-ku, Tokyo, 113-0033
}

Supporting Information

Table of Contents

Page

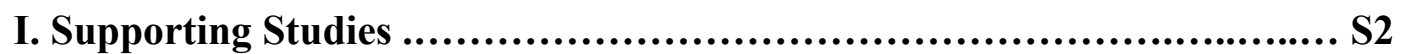

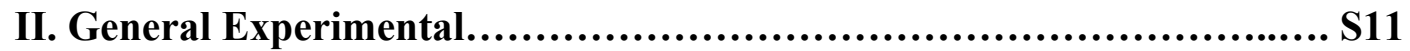

III. General Procedures............................................................

IV. Analytical Data for Allenyl and Homopropargyl Alcohols .................S14

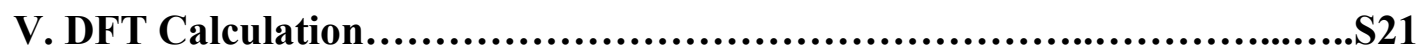

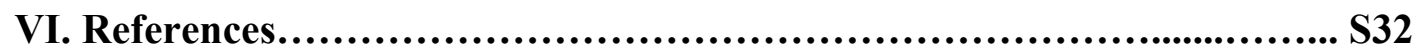

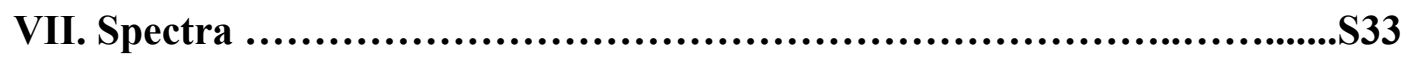




\section{Supporting Studies}

\section{Change of regioselectivity under different reaction conditions}

For the regioselective allenylation and propargylation using $\mathrm{Zn}(\mathrm{HMDS})_{2}$, the reactions were performed under the optimized reaction conditions. In the reaction systems, the allenylation products were obtained predominantly under the kinetic control conditions (at lower reaction temperature), while the propargylation products were obtained predominantly under the thermodynamic control conditions (in THF at higher reaction temperature and keeping low concentration of carbonyl compounds). As shown in Scheme S1, the regioselectivity could be controlled even by simply changing the reaction temperature and the reaction concentration of the ketone by slow addition. These results also suggested that the most important factor of the isomerization between the intermediates, propargyl and allenyl zinc species, was thermal stabilities of those zinc species.

Scheme S1. Allenylation/propargylation reactions in the same solvent (toluene)

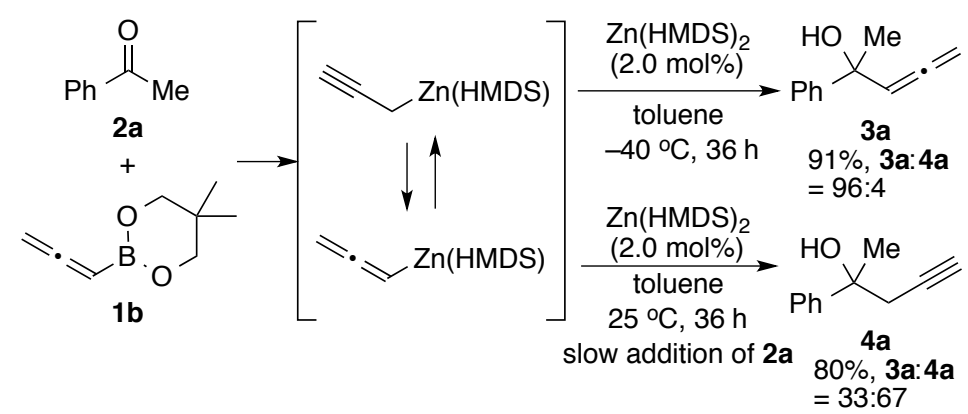

\section{NMR studies for mechanistic investigations}

To clarify the reaction mechanism, spectroscopic experiments were conducted in the stoichiomeric reaction of allenylboronate $\mathbf{1 b}$ with $\mathrm{Zn}(\mathrm{HMDS})_{2}$ by ${ }^{1} \mathrm{H} \mathrm{NMR}$ (Chart S1 and S2), ${ }^{13}$ C NMR (Chart S3 and S4) and ${ }^{11}$ B NMR (Chart S5) analyses (Scheme S2). These experiments were conducted in a dry, rubber septum-capped NMR tube. During NMR analyses, spin was used instead of stirring to keep the reactions in progress. Allenylboronate $\mathbf{1 b}(30.4 \mathrm{mg}, 0.200 \mathrm{mmol})$ was added dropwise into a solution of $\mathrm{Zn}(\mathrm{HMDS})_{2}(77.2 \mathrm{mg}, 0.200 \mathrm{mmol}, 100 \mathrm{~mol} \%)$ in toluene- $d_{8}(0.50 \mathrm{~mL})$ at $20{ }^{\circ} \mathrm{C}$ under an argon atmosphere. After mixing, the series of signals belonging to allenylboronate $\mathbf{1 b}$ and $\mathrm{Zn}(\mathrm{HMDS})_{2}$ gradually faded in the ${ }^{1} \mathrm{H}$ and ${ }^{13} \mathrm{C} \mathrm{NMR}$ analysis, and completely faded away after $2 \mathrm{~h}$ (Charts S1-S4). Instead, the new generated species appeared in solid state and exhibited very low solubility in toluene- $d_{8}$. In addition, various polar solvents were used to dissolve the solid such as THF, $\mathrm{CH}_{3} \mathrm{CN}$, DCM and DMF, but failed in the all cases. This insoluble solid might be an aggregated zinc species. Fortunately, this boron-to-zinc transmetallation process could be detected in ${ }^{11} \mathrm{~B}$ NMR analysis, wherein the initial signal of $\mathbf{1 b}$ at $25.8 \mathrm{ppm}$ progressively attenuated to transform into a new signal at $21.0 \mathrm{ppm}$, which is corresponding to boron amide 6 (Chart S5). Those results indicated that smooth transmetallation between $\mathbf{1 b}$ and $\mathrm{Zn}(\mathrm{HMDS})_{2}$ occurred, however, it was still unclear which kind of zinc species formed on this stage. 
Scheme S2. NMR experiment using $\mathrm{Zn}(\mathrm{HMDS})_{2}$

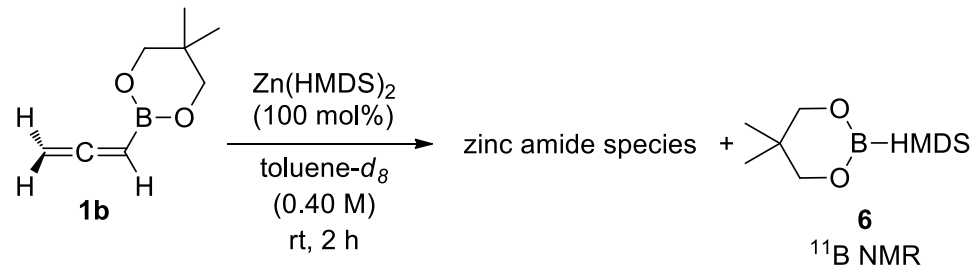

Chart S1 ${ }^{1} \mathrm{H}$ NMR experiments using $\mathrm{Zn}(\mathrm{HMDS})_{2}$ and $\mathbf{1 b}(1: 1)$

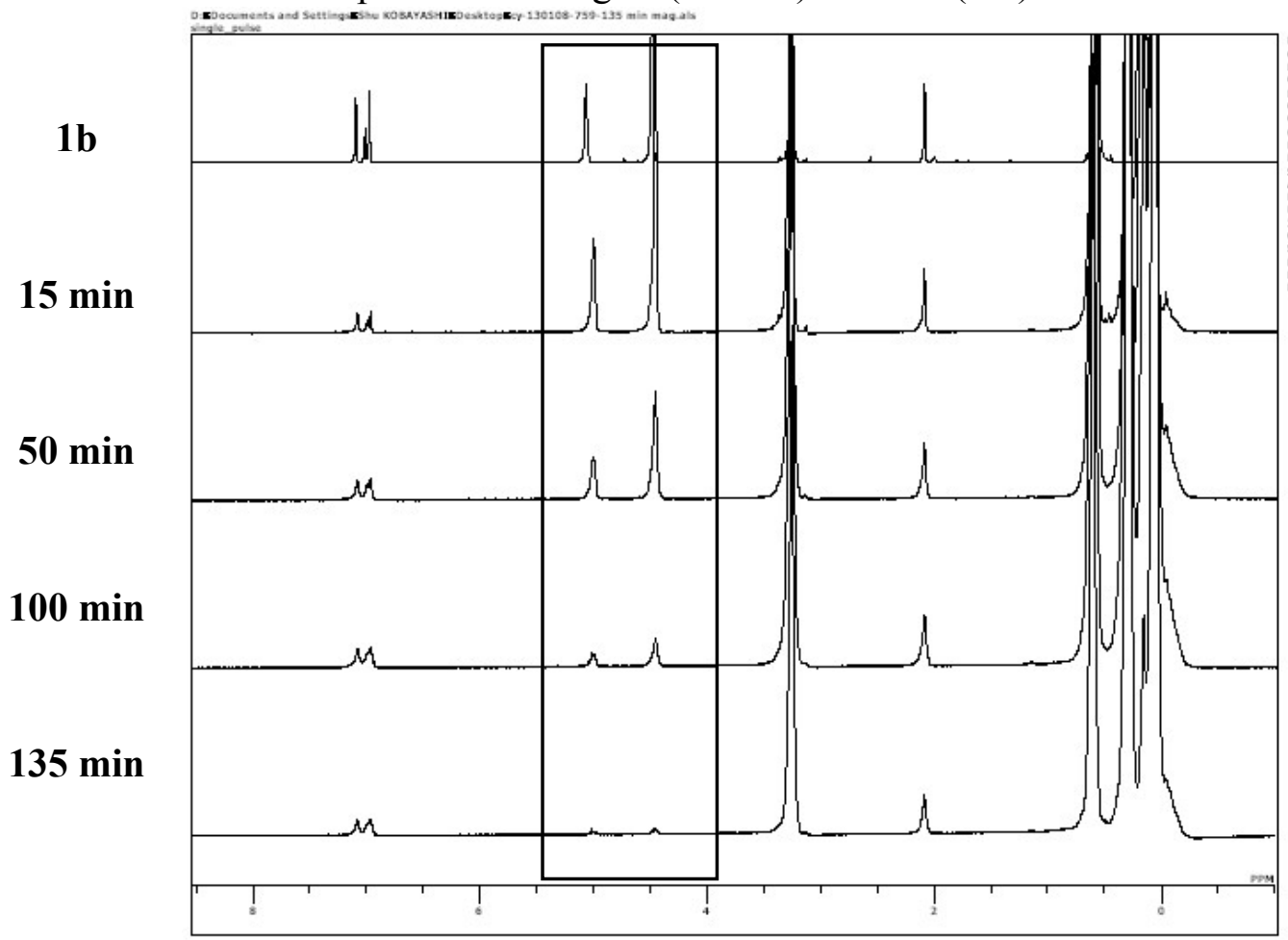

Chart S2 ${ }^{1} \mathrm{H}$ NMR experiments using $\mathrm{Zn}(\mathrm{HMDS})_{2}$ and $\mathbf{1 b}(1: 1)$ (magnifying the upfield)

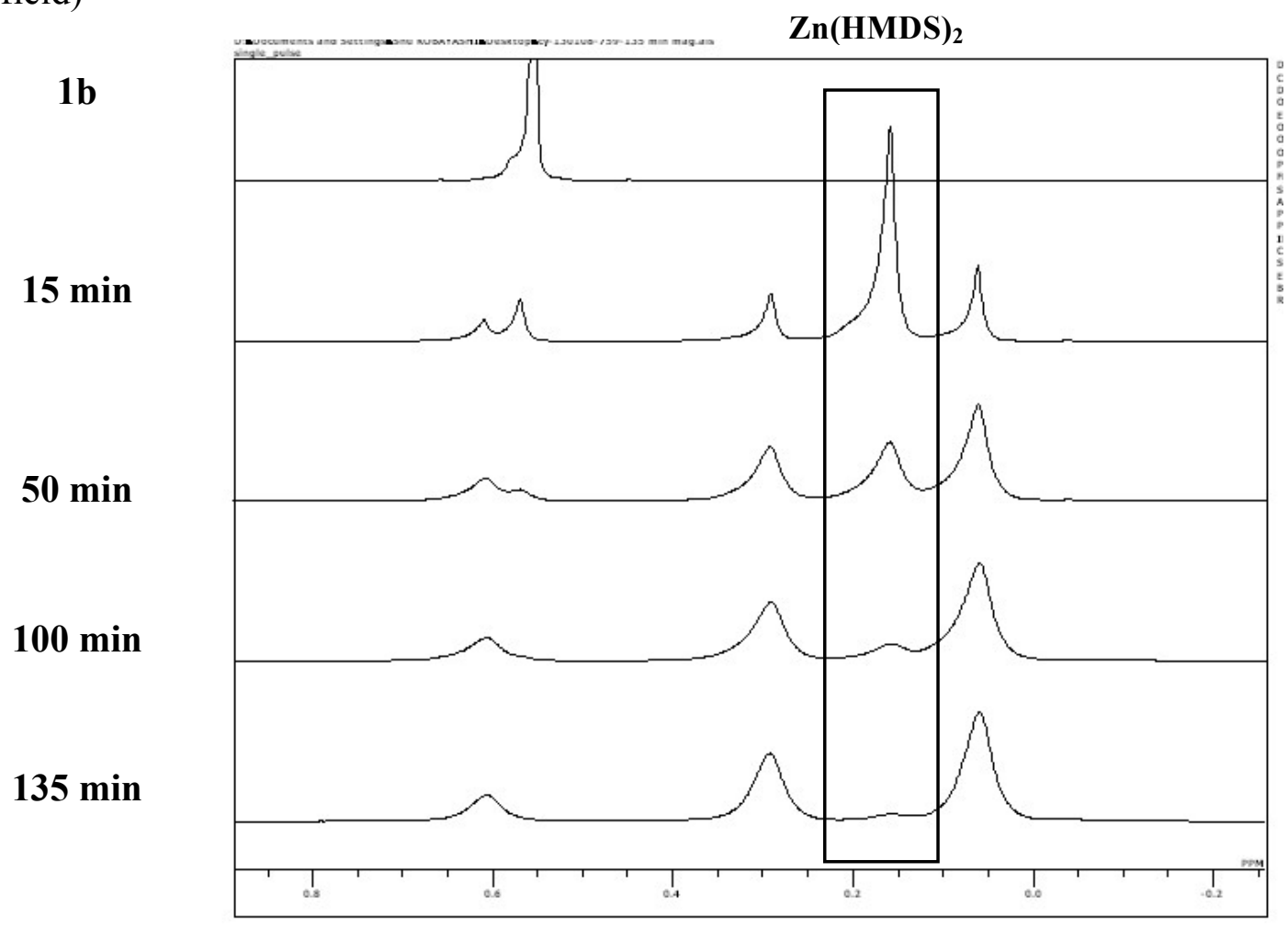


Chart S3 ${ }^{13} \mathrm{C}$ NMR experiments using $\mathrm{Zn}(\mathrm{HMDS})_{2}$ and $\mathbf{1 b}(1: 1)$

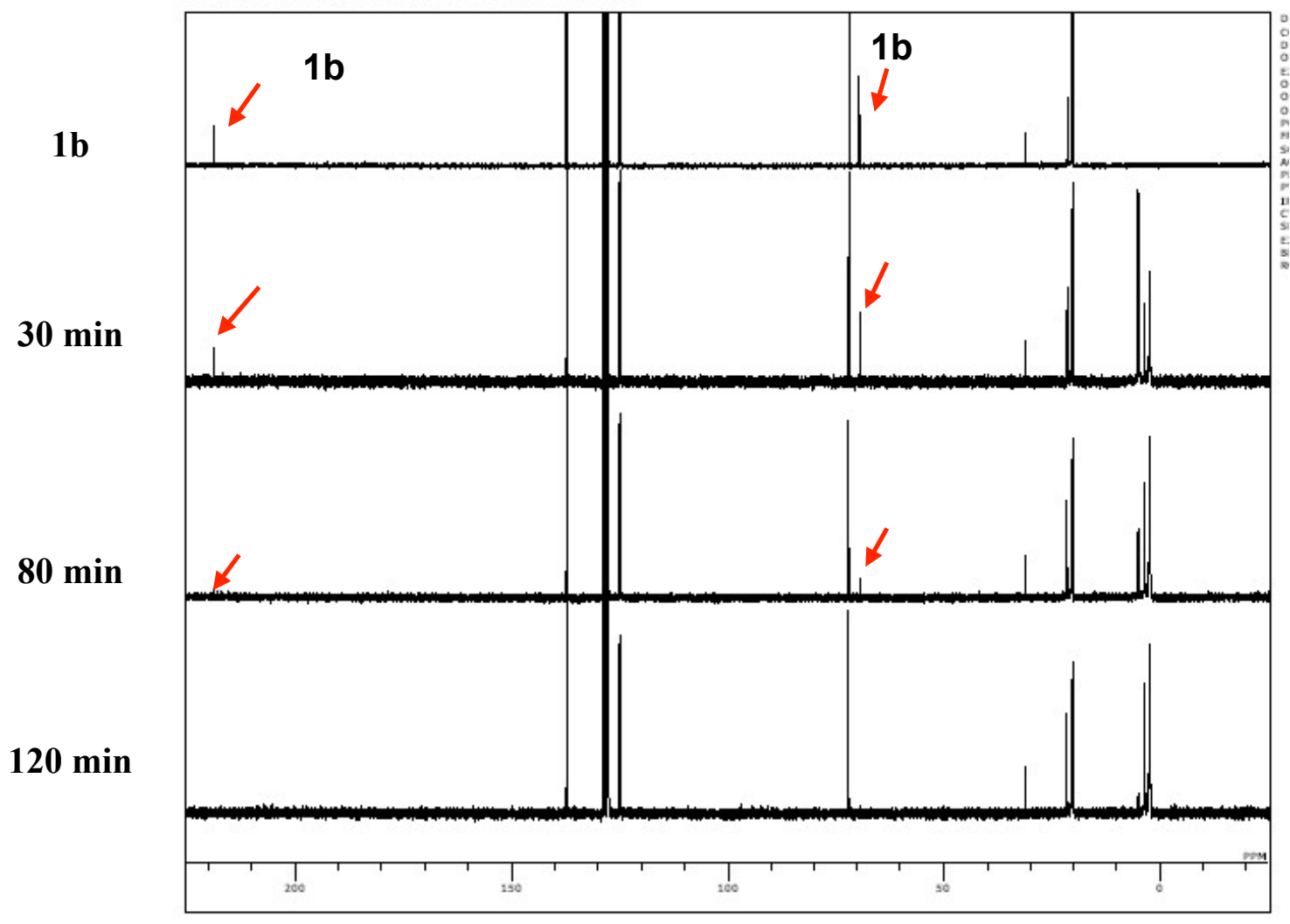

Chart S4 ${ }^{13} \mathrm{C}$ NMR experiments using $\mathrm{Zn}(\mathrm{HMDS})_{2}$ and $\mathbf{1 b}$ (1:1) (magnifying the upfield)

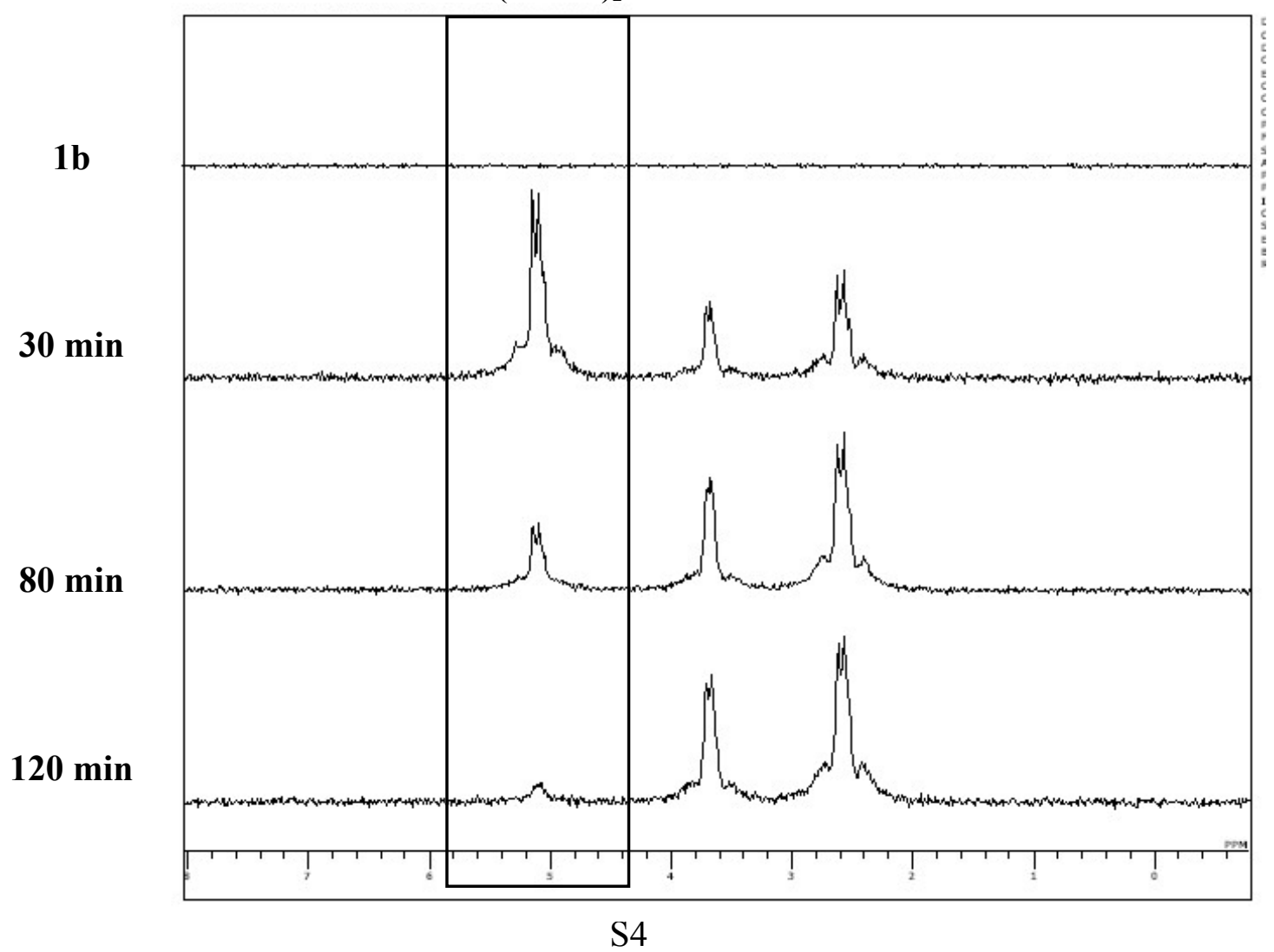


Chart S5 ${ }^{11} \mathrm{~B}$ NMR experiments using Zn(HMDS) $)_{2}$ and $\mathbf{1 b}(1: 1)$

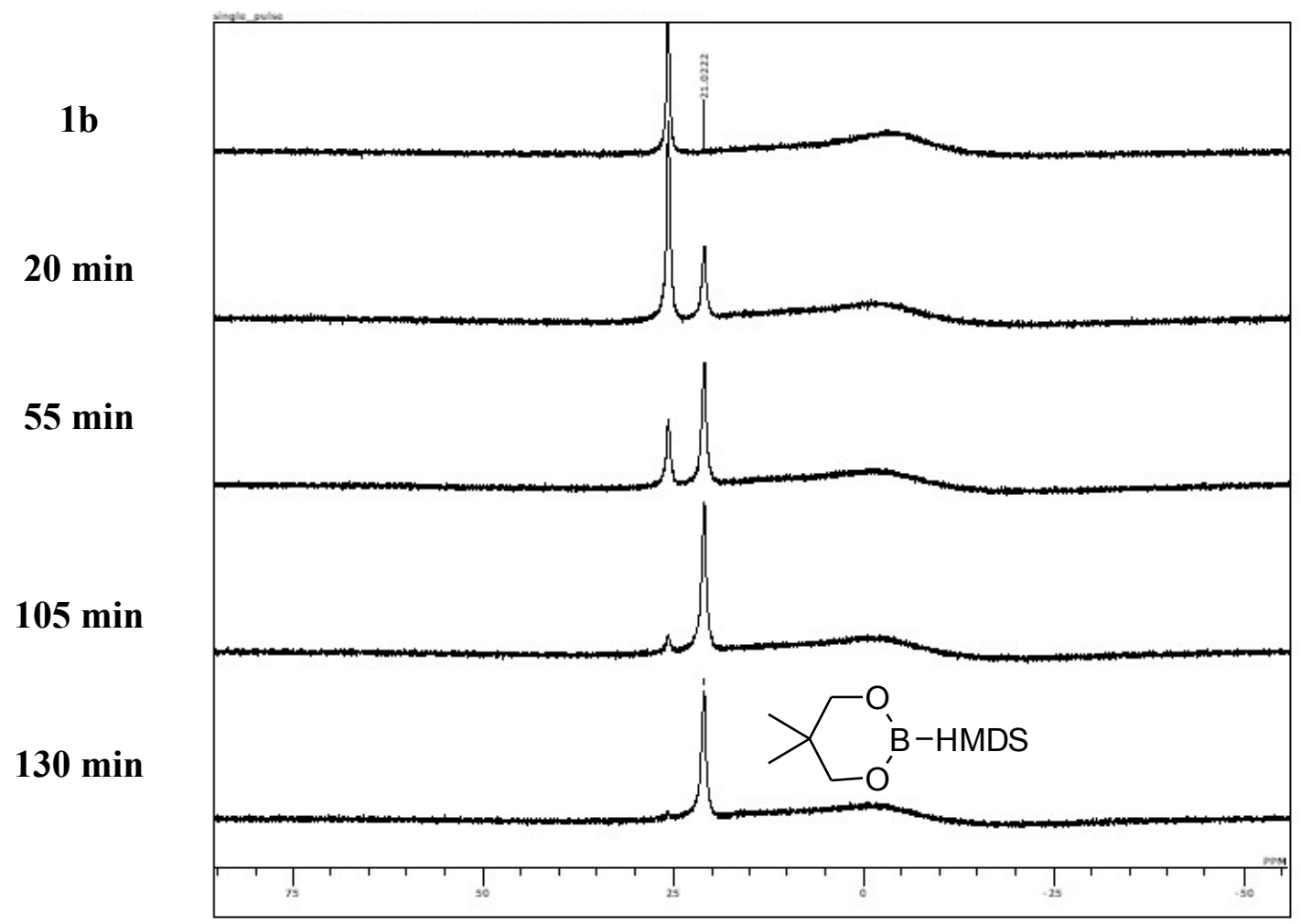

Next, we tried to see which zinc species formed on the transmetallation stage. To obtain further information by NMR analyses, soluble zinc species is required. We decided to use the chiral diamine ligand $\mathbf{L 1}$ to avoid the insoluble solid formation of the zinc species by coordination. $\quad$ L1 $(39.3 \mathrm{mg}, 0.100 \mathrm{mmol})$ and $\mathrm{Zn}(\mathrm{HMDS})_{2}$ $(38.6 \mathrm{mg}, 0.100 \mathrm{mmol})$ were combined in a dry toluene- $d_{8}(1.00 \mathrm{~mL})$, and then heated at $75{ }^{\circ} \mathrm{C}$ for $2 \mathrm{~h}$ (Scheme S3). The deprotonation of $\mathbf{L 1}$ by $\mathrm{Zn}(\mathrm{HMDS})_{2}$ (which has been confirmed by the formation of H-HMDS during the process), provide a chiral zinc amide preliminarily. ${ }^{1}$ Subsequently, $\mathbf{1 b}(15.2 \mathrm{mg}, 0.100 \mathrm{mmol})$ was added just before the NMR monitoring. Under the current condition, gradually boron to zinc exchange was observed in ${ }^{1} \mathrm{H}$ NMR (Chart S6), ${ }^{13} \mathrm{C}$ NMR (Chart S7) and ${ }^{11} \mathrm{~B}$ NMR analyses (Chart S8). The series of signals belonging to $\mathbf{1 b}$ gradually faded. The emerged three sets of signals at $2.21,2.02$, and $1.90 \mathrm{ppm}$ in the ${ }^{1} \mathrm{H}$ NMR analysis were assigned as the signals of propargyl zinc species. The intensities of these signals increased with time, and after 70 minutes, full conversion of $\mathbf{1 b}$ was observed. Moreover, two new signals around 80-70 ppm and one broad peak around 3-2 ppm emerged in the ${ }^{13} \mathrm{C}$ NMR analysis, respectively, which were represented on the typical region of propargylmetal species in NMR spectroscopies. ${ }^{2}$ Regarding the ${ }^{11} \mathrm{~B}$ NMR

Scheme S3 NMR experiment using Zn(HMDS) $)_{2}$ and L1

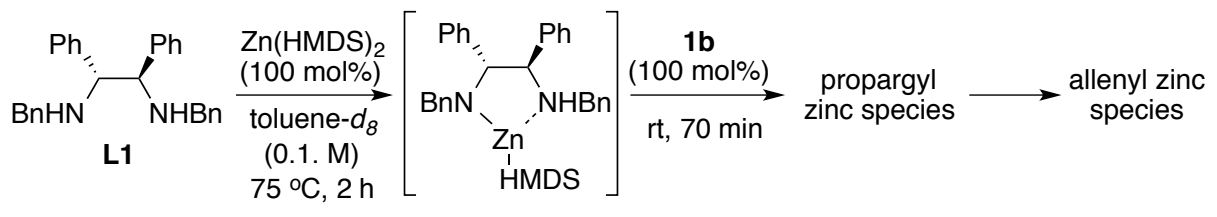


Chart S6 ${ }^{1} \mathrm{H}$ NMR experiments using Zn(HMDS) $)_{2}$ L1 and 1b (1:1:1)

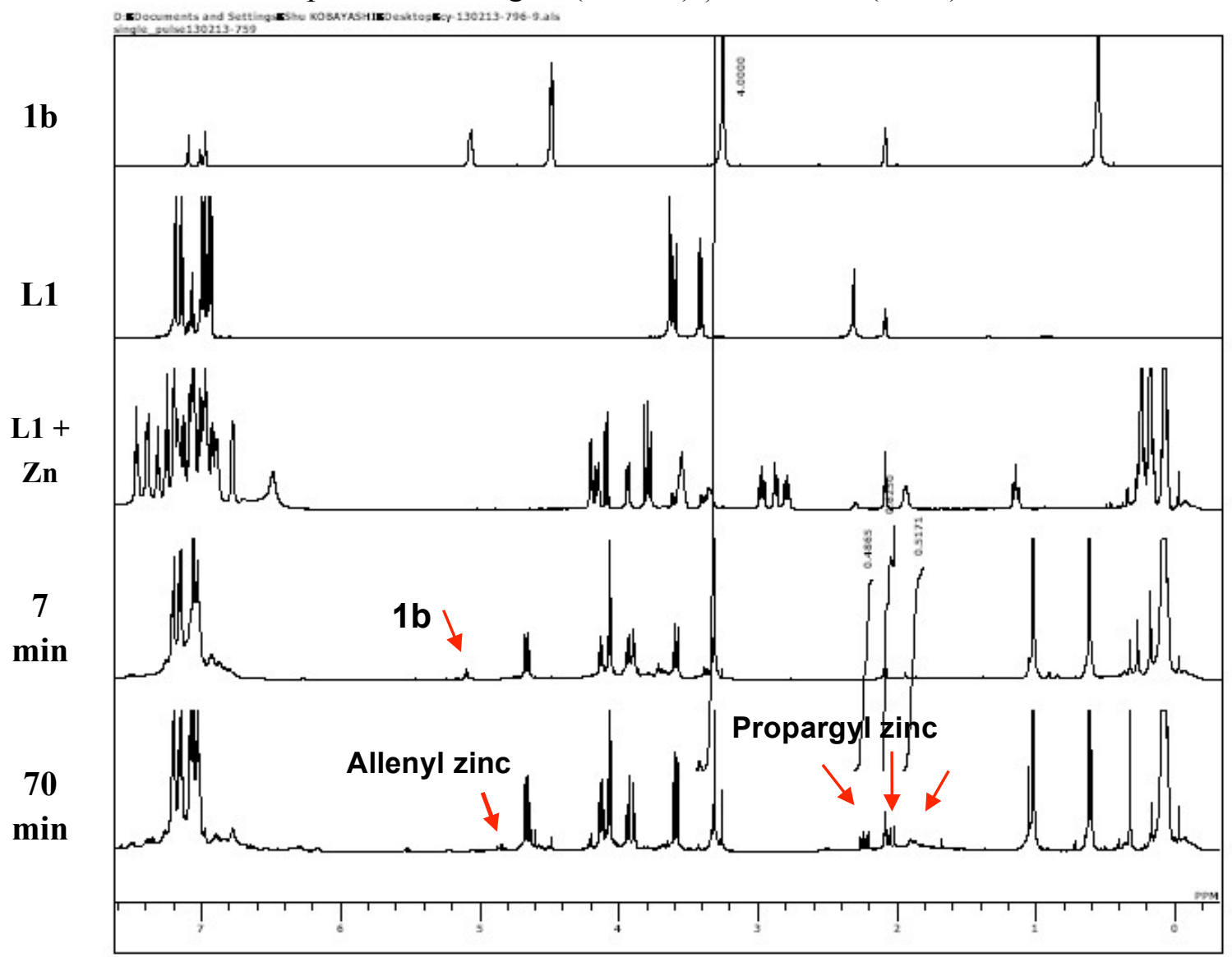

Chart $\mathbf{S 7}{ }^{13} \mathrm{C}$ NMR experiments using Zn(HMDS) $)_{2}$, L1 and $\mathbf{1 b}(1: 1: 1)$

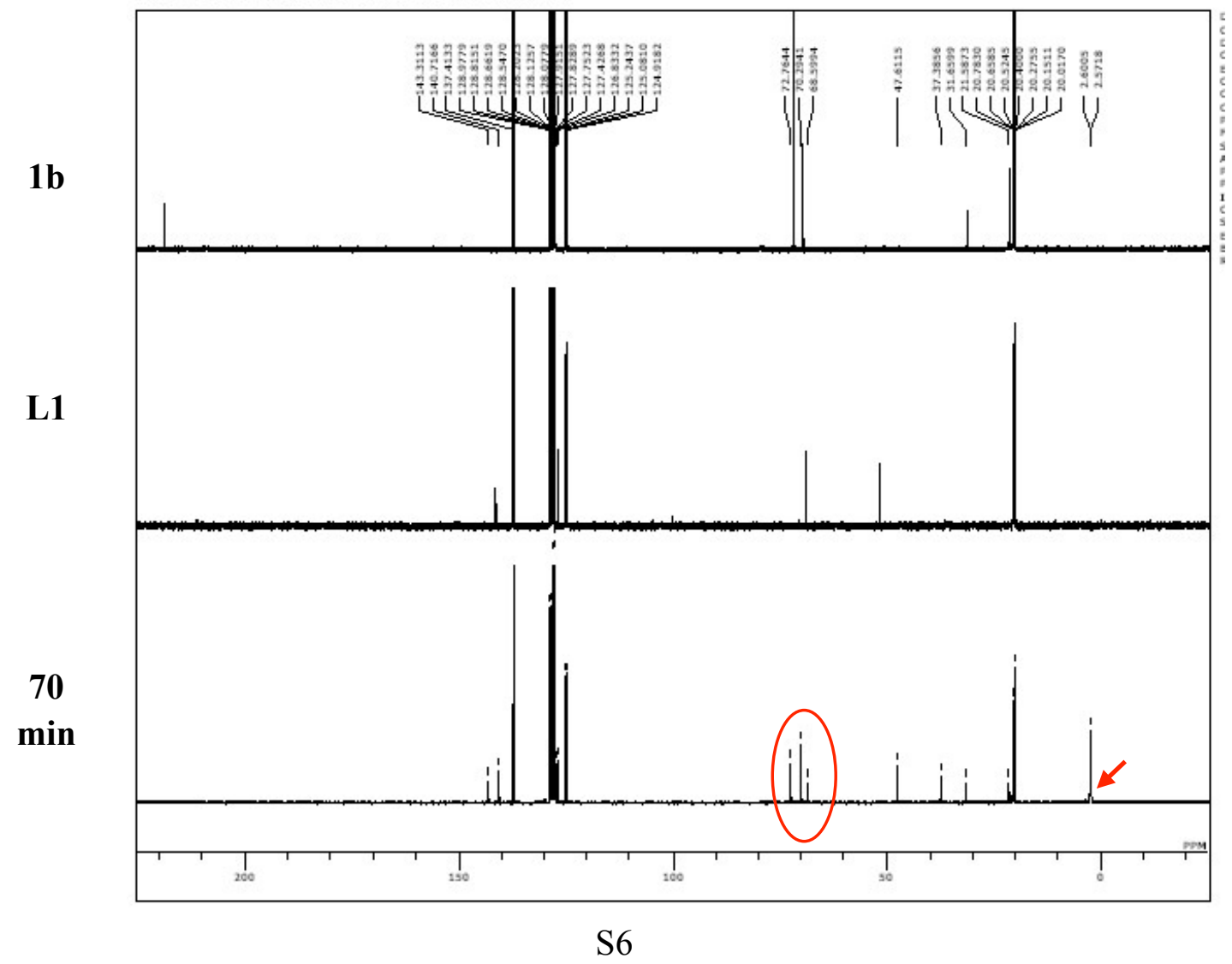


Chart S8 ${ }^{11} \mathrm{~B}$ NMR experiments using Zn(HMDS) $)_{2}$ L1 and $\mathbf{1 b}(1: 1: 1)$

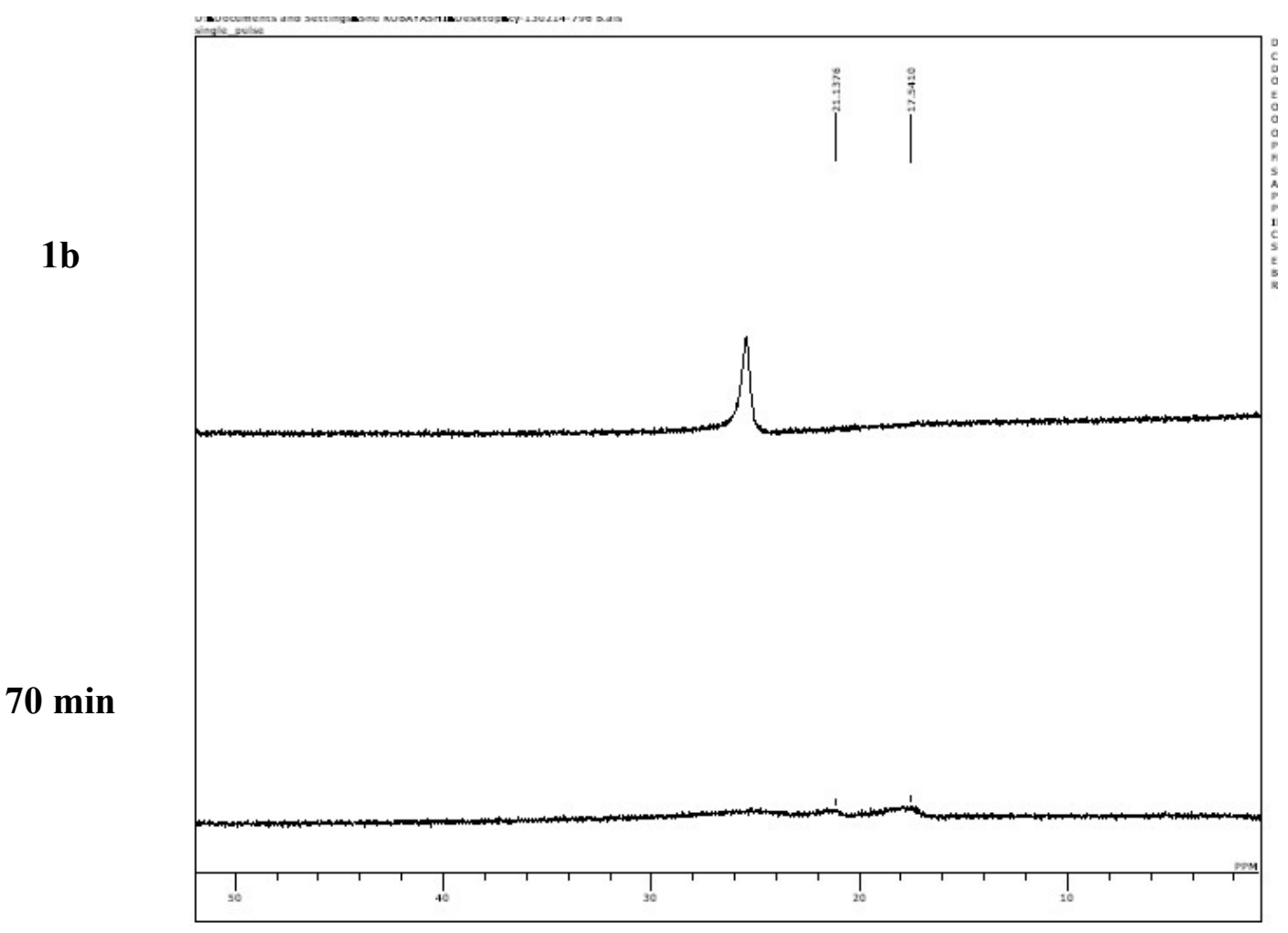

analysis, two broad peaks at 21.1 and $17.5 \mathrm{ppm}$ replaced with the signal of $\mathbf{1 b}$ at 25.4 ppm after 70 minutes. The peak at $21.1 \mathrm{ppm}$ should be corresponding to boron amide 6; another peak might be derived from a certain coordination of boron compound to $\mathbf{L 1}$ but it was not clear at this stage. It should be noted that propargyl zinc species clearly formed by transmetallation just after mixing $\mathbf{1 b}$ with $\mathrm{Zn}(\mathrm{HMDS})_{2}$.

On the other hand, during the experiments, two sets of tiny signals around 5.0-4.8 ppm and 4.3-4.1 ppm were also observed in the ${ }^{1} \mathrm{H}$ NMR analysis (Chart S9) after 100 minutes, which were corresponding to the signals of allenyl zinc, generated from the isomerization of propargyl zinc. The intensities of these signals increased with time and after $42 \mathrm{~h}$, allenyl zinc species became dominant over propargyl zinc. After $66 \mathrm{~h}$, high abundance of allenyl zinc was observed, in contrast, trace signals of propargyl zinc remained in the ${ }^{1} \mathrm{H}$ NMR analysis. Those results indicated that smooth isomerization from propargyl zinc species to allenyl one occurred easily in toluene at rt. It should be noted that direct observation of the isomerization from propargyl zinc to allenyl one is remarkable. 
Chart S9 ${ }^{1} \mathrm{H}$ NMR experiments concerning isomerization of propargyl zinc

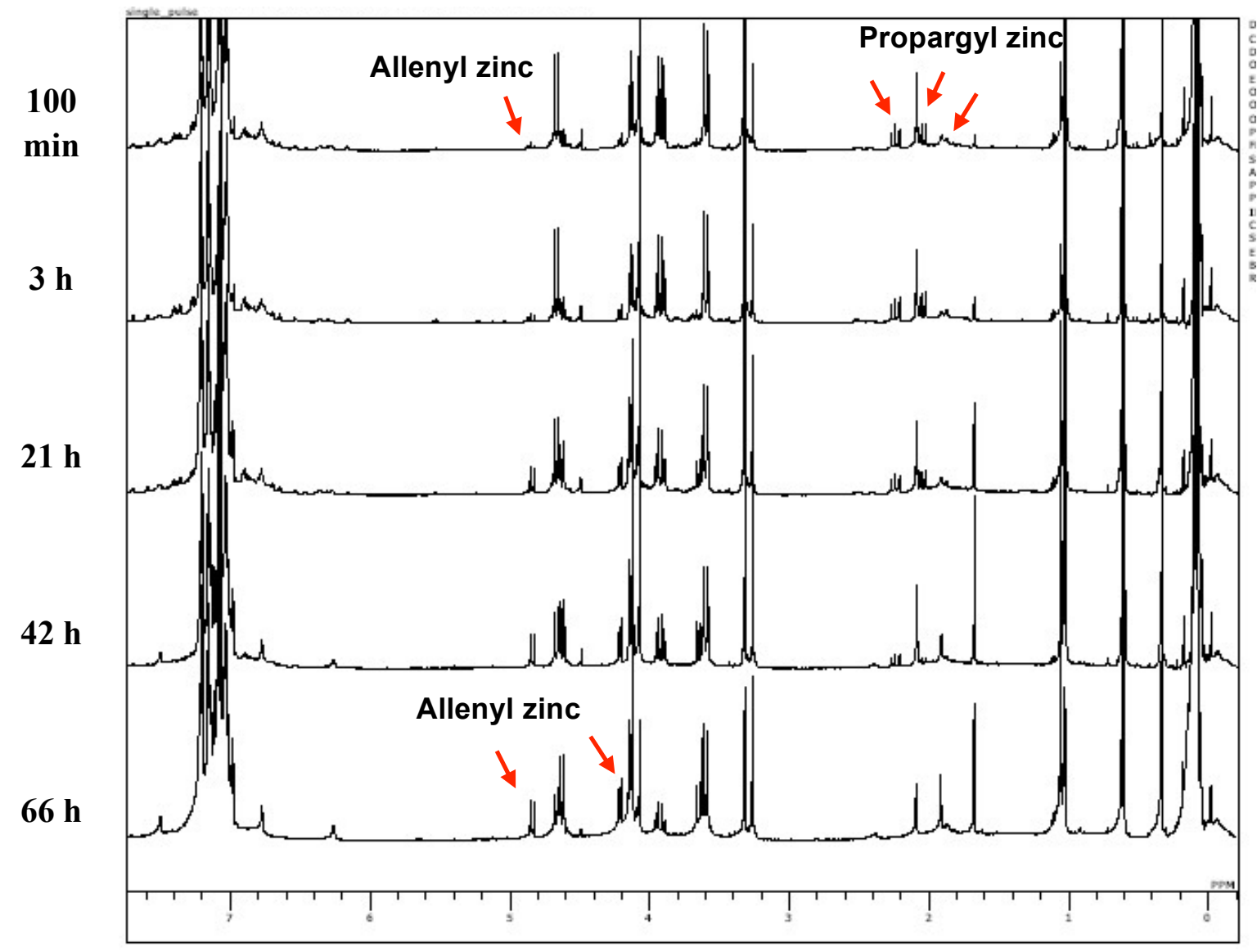

Additionally, the reaction of $\mathbf{1 b}(30.4 \mathrm{mg}, 0.200 \mathrm{mmol})$ with $\mathrm{ZnEt}_{2}(182 \mu \mathrm{L}, 1.1$ $\mathrm{M}$ in toluene, $0.200 \mathrm{mmol})$ in dry toluene- $d_{8}(0.40 \mathrm{M})$ was also monitored by ${ }^{1} \mathrm{H}$ NMR and ${ }^{11} \mathrm{~B}$ NMR analyses (Chart S10 and S11, Scheme S4). $\mathrm{ZnEt}_{2}$ is known to be a good catalyst in allenlylation and propargylation reactions of ketones with allenyl and propargyl boronates. ${ }^{3}$ It was found that the diminution of $\mathbf{1 b}$ was very slow, and after $6 \mathrm{~h}$, the majority of $\mathbf{1 b}$ remained. Moreover, ${ }^{11} \mathrm{~B}$ NMR spectroscopy appears quite messy. Those results indicated that transmetallation between allenyl boronate $\mathbf{1 b}$ and $\mathrm{ZnEt}_{2}$ was very slow compared with the case of $\mathrm{Zn}(\mathrm{HMDS})_{2}$.

Scheme S4 NMR experiment using $\mathrm{ZnEt}_{2}$

$$
\begin{aligned}
& 1 \mathrm{~b}
\end{aligned}
$$

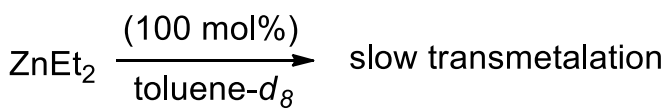

$$
\begin{aligned}
& \text { (0.40 M) } \\
& \mathrm{rt}
\end{aligned}
$$


Chart S10 ${ }^{1} \mathrm{H}$ NMR experiments using $\mathrm{ZnEt}_{2}$ and $\mathbf{1 b}(1: 1)$

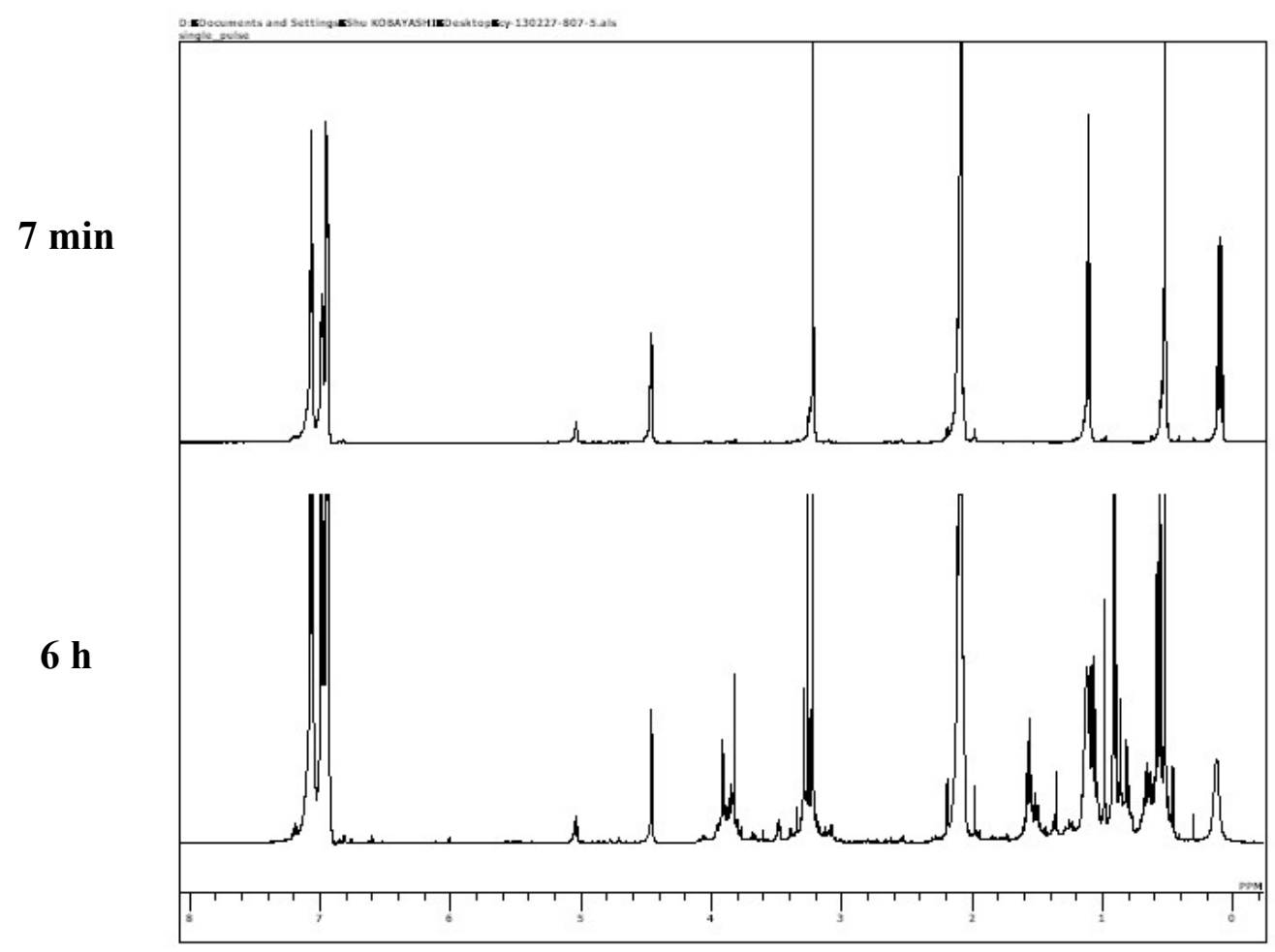

Chart S11 ${ }^{11} \mathrm{~B}$ NMR experiments using $\mathrm{ZnEt}_{2}$ and $\mathbf{1 b}(1: 1)$

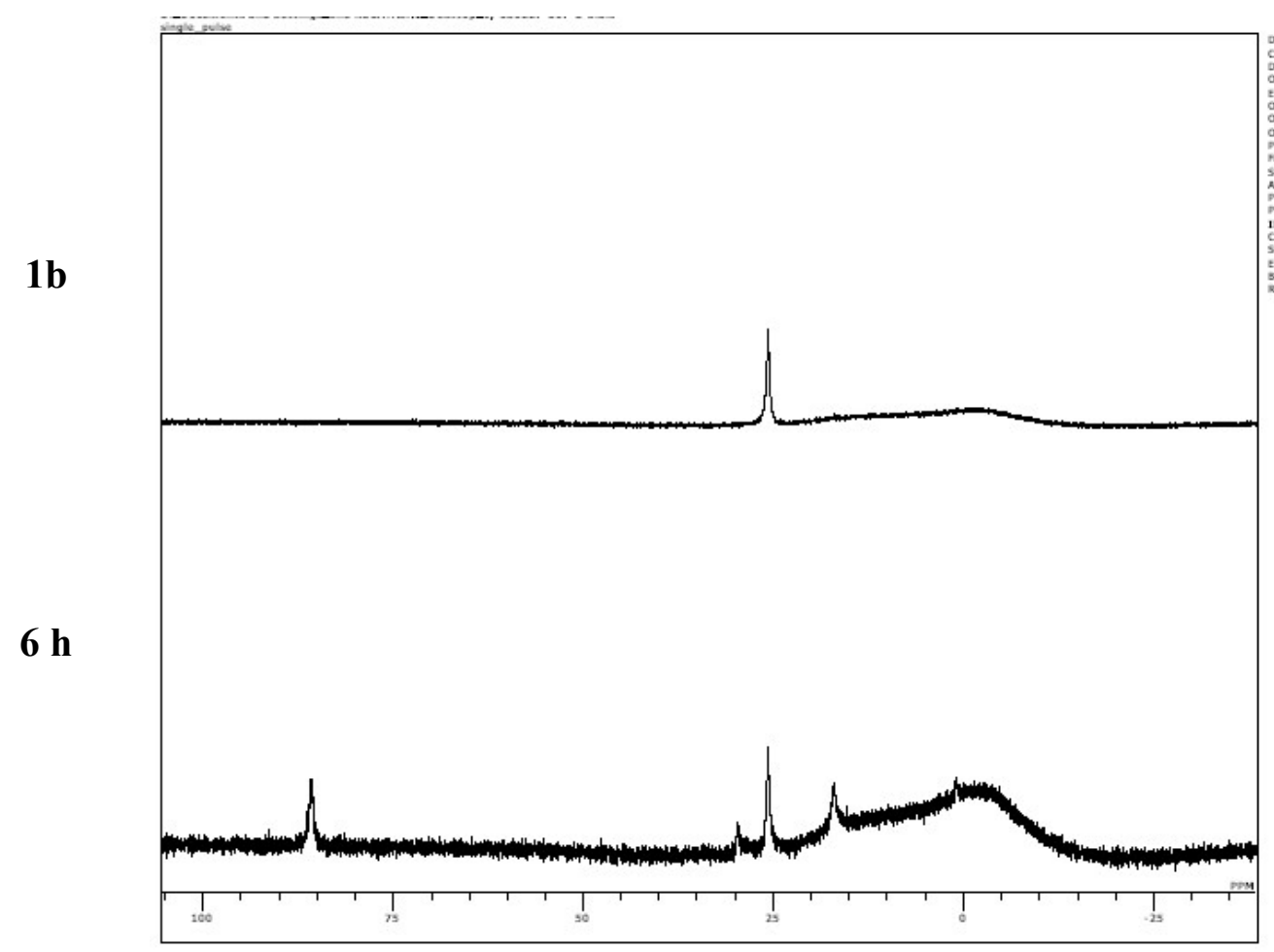




\section{DFT calculation of allenyl and propargyl zinc species}

DFT calculation of energy levels of the proposed intermediates was performed (Figure S1). Comparison of the energies of the allenyl zinc species and the propargyl zinc species indicated that the allenyl zinc species was more stable than propargyl zinc species $(6.4 \mathrm{kcal} / \mathrm{mol})$. These results suggested that the propargyl zinc species could isomerize to the allenyl zinc species under thermodynamic control conditions. The energies of the transition states of the allenylation and the propargylation of a ketone (acetone) was also calculated, and it was suggested that the transition state energy of the allenylation reaction from the propargyl zinc species was lower than that of the propargylation reaction from the allenylzinc species (allenylation: $10.0 \mathrm{kcal} / \mathrm{mol}$; propargylation: $15.6 \mathrm{kcal} / \mathrm{mol}$ ), which means that once the propargyl zinc species forms in the reaction system, the successive allenylation reaction can occur more smoothly than the propargylation reaction. Mechanism of the isomerization between the propargyl zinc species and the allenyl zinc species is still not clear; however, high reaction temperature might enhance the isomerization of the propargyl zinc species to more stable allenyl zinc species, and slow addition of a ketone might help the isomerization before the addition to a ketone by keeping the concentration of the electrophile lower.

Figure S1. DFT calculation of the proposed intermediates
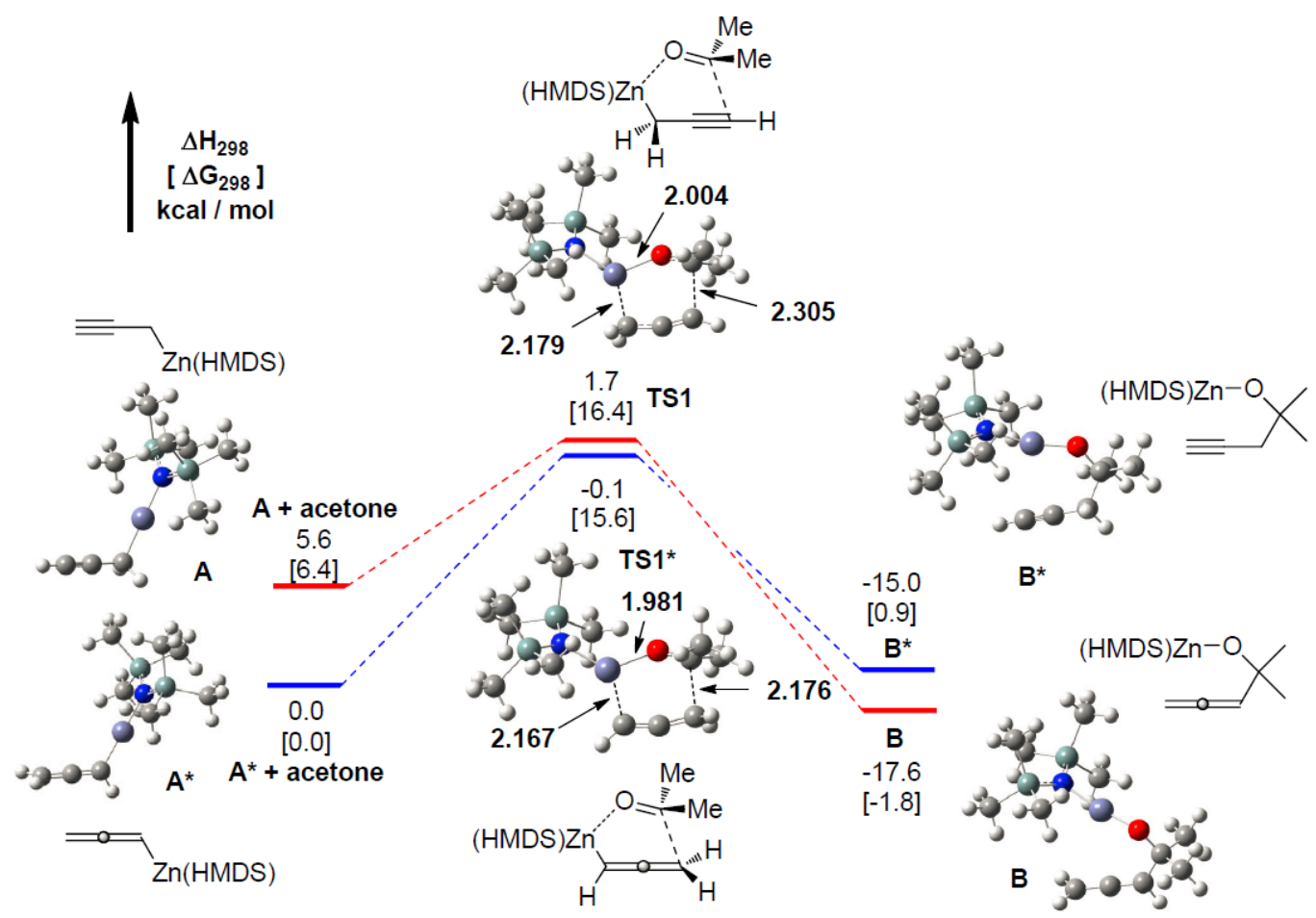


\section{General Experimental}

NMR spectra were recorded on a JEOL ECX-400, a JEOL ECA-500, or a JEOL ECX-600 spectrometer, operating at $400 \mathrm{MHz}, 500 \mathrm{MHz}$, or $600 \mathrm{MHz}$ for ${ }^{1} \mathrm{H} \mathrm{NMR}$, $100 \mathrm{MHz}, 125 \mathrm{MHz}$, or $150 \mathrm{MHz}$ for ${ }^{13} \mathrm{C} \mathrm{NMR}$ and $192 \mathrm{MHz}$ for ${ }^{11} \mathrm{~B} \mathrm{NMR}$. Chemical shifts were reported downfield from tetramethylsilane (TMS) or in the scale relative to the corresponding solvent used as an internal reference. Infra Red (IR) spectra were measured using a JASCO FT/IR-4200 spectrometer. High Resolution Mass Spectra (HRMS) were recorded using a JEOL JMST100TD (DART) spectrometer. High-performance liquid chromatography was carried out using following apparatuses; SHIMADZU LC-20AB (liquid chromatograph), SHIMADZU SPD-M20A (Photo diode array detector) and DGU-20A 3 . Preparative thin-layer chromatography (PTLC) was carried out using Wakogel B-5F from Wako Pure Chemical Industries, Ltd. Flash column chromatography was performed over silica gel $60 \mathrm{~N}$ from Kanto Chemical Co., Inc.

All solvents used were commercially available dry solvents that were further dried and degassed appropriately under an argon atmosphere, and stored over activated molecular sieves in an argon box prior to use. Unless otherwise specified, all aldehydes and ketones (purchased from commercial sources) used in this work were distilled under an argon atmosphere or recrystallized prior to use. Pinacol allenylboronate $\mathbf{1 a}$ and allenyl boronate $\mathbf{1} \mathbf{b}^{4}$ were prepared by reported methods; their analyses are in agreement with the reported data. Zinc bis[bis(trimethylsilyl)amide] $\left(\mathrm{Zn}(\mathrm{HMDS})_{2}\right)^{5}$ was prepared according to a reported procedure, and stored in glove box at $-30{ }^{\circ} \mathrm{C}$. Chiral ligand $\mathbf{L 1}^{6}$ was prepared according to a literature procedure. All reactions were carried out under an argon atmosphere in flame-dried glassware. References following the compound names indicate the corresponding literature articles where ${ }^{1} \mathrm{H}$ and ${ }^{13} \mathrm{C}$ NMR data have previously been reported.

\section{General Procedure}

\section{Typical Procedure for $\mathrm{Zn}(\mathrm{HMDS})_{2}$-Catalyzed Allenylation of Carbonyl Compounds 2a-t with Allenylboronate 1b}

$\mathrm{Zn}$ (HMDS $)_{2}(0.0080 \mathrm{mmol})$ was dissolved in dry toluene $(0.2 \mathrm{~mL})$, and then the solution was cooled to $-40{ }^{\circ} \mathrm{C}$ or $-60{ }^{\circ} \mathrm{C}$ (depending on the substrates). To this solution was added dropwise the solution of allenylboronate $1(0.44 \mathrm{mmol})$ in dry toluene $(0.40 \mathrm{~mL})$, and was then added dropwise a solution of $2(0.40 \mathrm{mmol})$ in dry toluene $(0.40 \mathrm{~mL})$. When the reaction was conducted in $8.0 \mathrm{mmol}$ scale, $\mathrm{Zn}(\mathrm{HMDS})_{2}$ and $\mathbf{1 b}$ were mixed at $0{ }^{\circ} \mathrm{C}$, and after cooled at $-60{ }^{\circ} \mathrm{C}$, 2a was added. The mixture was stirred at the same temperature for $36 \mathrm{~h}$. Then the reaction was quenched with saturated aqueous $\mathrm{NH}_{4} \mathrm{Cl}$. The resultant mixture was allowed to be warmed up to room temperature and extracted with EtOAc $\left(10 \mathrm{~mL}^{*} 3\right)$, and the combined organic layers were dried over anhydrous $\mathrm{Na}_{2} \mathrm{SO}_{4}$. The solvent was evaporated in vacuo, and the residue was purified by silica gel column chromatography or preparative thin-layer chromatography to afford the corresponding allenyl alcohols or mixture of 
allenylic/propargyl alcohols.

\section{Typical Procedure for $\mathrm{Zn}(\mathrm{HMDS})_{2}$-Catalyzed Propargylation of Carbonyl Compounds 2a-t with Allenylboronate 1b}

$\mathrm{Zn}(\mathrm{HMDS})_{2}(0.0012 \mathrm{mmol})$ was dissolved in freshly distilled dry THF $(0.2 \mathrm{~mL})$, then the solution was keep in 15 or $25{ }^{\circ} \mathrm{C}$ (depending on the substrates). To this solution was added a solution of allenylboronate $1(0.600 \mathrm{~mol})$ in freshly distilled dry THF $(0.4 \mathrm{~mL})$. After stirring for $10 \mathrm{~min}$, the solution of $2(0.40 \mathrm{mmol})$ in freshly distilled dry THF $(0.4 \mathrm{~mL})$ was then slowly added during $12 \mathrm{~h}$. The mixture was then stirred at this temperature for another $24 \mathrm{~h}$. Finally, the reaction was quenched by saturated aqueous $\mathrm{NH}_{4} \mathrm{Cl}$, and the resultant mixture was extracted by EtOAc (10 $\mathrm{mL} * 3$ ). The combined organic layer was dried over anhydrous $\mathrm{Na}_{2} \mathrm{SO}_{4}$. The solution was evaporated in vacuum, and the residue was purified by silica-gel column chromatography or preparative thin-layer chromatography to afford the desired product as mixture of allenylic/propargyl alcohols.

\section{Typical Procedure for Asymmetric Allenylation of 2n with Allenylboronate 1b} (Scheme 5)

$\mathrm{Zn}$ (HMDS $)_{2}(0.0200 \mathrm{mmol})$ was dissolved in dry toluene $(0.2 \mathrm{~mL})$, and then the solution of chiral ligand $\mathbf{L 1}(0.024 \mathrm{mmol})$ in toluene $(0.3 \mathrm{~mL})$ was added. The mixture was stirred at $75^{\circ} \mathrm{C}$ for $1 \mathrm{~h}$, and the color of the solution gradually changed to red. After that, the solution was cooled to $-40{ }^{\circ} \mathrm{C}$, and allenyl boronate $\mathbf{1 b}(0.44$ mmol) and a solution of $\mathbf{2 n}(0.40 \mathrm{mmol})$ in dry toluene $(0.5 \mathrm{~mL})$ were successively added dropwise. The mixture was stirred at $-40{ }^{\circ} \mathrm{C}$ for $36 \mathrm{~h}$. The reaction was quenched with saturated aqueous $\mathrm{NH}_{4} \mathrm{Cl}$. The resultant mixture was allowed to be warmed up to room temperature and extracted with EtOAc $(3 * 10 \mathrm{~mL})$, and the combined organic layer was dried over anhydrous $\mathrm{Na}_{2} \mathrm{SO}_{4}$. The solvent was evaporated in vacuo, and the residue was purified by preparative thin-layer chromatography to afford the desired product.

\section{Typical Procedure for Asymmetric propargylation of 2c with Allenylboronate 1b} (Scheme 5)

Inda-Box $(0.024 \mathrm{mmol})$ was dissolved in dry DCE $(0.1 \mathrm{~mL})$, and then the solution was keep at rt. To this solution was quickly added a solution of $\mathrm{Zn}(\mathrm{HMDS})_{2}$ $(0.020 \mathrm{mmol})$ in DCE $(0.1 \mathrm{~mL})$ under stirring. After stirring for $20 \mathrm{~min}$, the solution was cool down to $-40{ }^{\circ} \mathrm{C}$. To this solution was successively added the solution of allenylboronate $(0.44 \mathrm{~mol})$ in dry DCE $(0.4 \mathrm{~mL})$ and $2 \mathrm{c}(0.40 \mathrm{mmol})$ in dry DCE $(0.4$ $\mathrm{mL})$. The mixture was then stirred at $-40{ }^{\circ} \mathrm{C}$ for $36 \mathrm{~h}$. Finally, the reaction was quenched with saturated aqueous $\mathrm{NH}_{4} \mathrm{Cl}$, and the resultant mixture was extracted by EtOAc $(3 * 10 \mathrm{~mL})$. The combined organic layer was dried over anhydrous $\mathrm{NaSO}_{4}$. The solution was evaporated in vacuum, and the residue was purified by thin-layer chromatography to afford the desired product. 


\section{Procedure for Preparation of Boron Amide $6^{7}$}

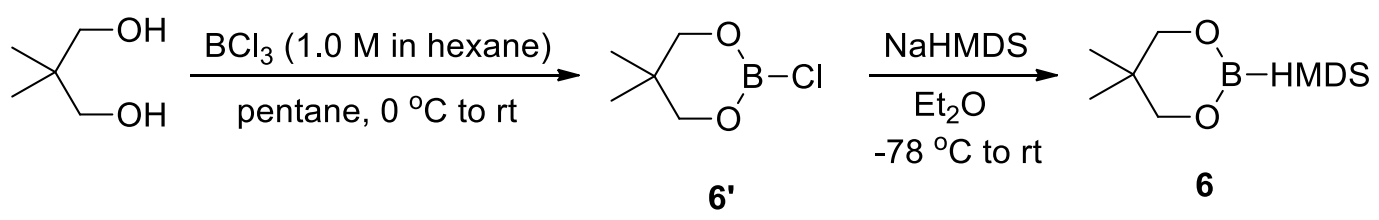

A solution of 2,2-dimethylpropane-1,3-diol (3.00 g, $28.5 \mathrm{mmol})$ in pentane (100 $\mathrm{mL}$ ) in a $200 \mathrm{~mL}$ three-necked round-bottom flask (argon, dropping funnel, blubber with hose and funnel (outlet of $\mathrm{HCl}$ into a solution of $\mathrm{NaOH}$ ) was cooled to $0{ }^{\circ} \mathrm{C}$. To this solution was added dropwise $\mathrm{BCl}_{3}(1 \mathrm{M}$ in hexane, $30 \mathrm{~mL}, 30 \mathrm{mmol})$ during $2 \mathrm{~h}$. The reaction mixture was stirred for $1 \mathrm{~h}$ at room temperature. The resultant mixture was concentrated in vacuo, and the residue was distilled to give 6' $(1.82 \mathrm{~g}, 43 \%)$ as a colorless liquid (bp. 50-60 ${ }^{\circ} \mathrm{C} / 10$ mbar). ${ }^{1} \mathrm{H}$ NMR $\left(\mathrm{CDCl}_{3}, 500 \mathrm{MHz}\right) \delta: 3.69-3.61$ (m, $4 \mathrm{H}), 0.95-0.91(\mathrm{~m}, 6 \mathrm{H})$.

To a solution of 6' $(1.82 \mathrm{~g}, 12.2 \mathrm{mmol})$ in dry diethyl ether $(10 \mathrm{~mL})$ cooled to -78 ${ }^{\circ} \mathrm{C}$ with a cooling bath, the solution of NaHMDS (2.24 g, $\left.12.2 \mathrm{mmol}\right)$ in diethyl ether $(10 \mathrm{~mL})$ was added dropwise. The reaction mixture was allowed to be warmed up slowly to room temperature and stirred overnight under argon atmosphere. The flask was brought into a glove box fulfilled with Ar, and the precipitate was filtered through dry celite. The collected solid was washed with dry $\mathrm{Et}_{2} \mathrm{O}\left(10 \mathrm{~mL}^{* 2}\right)$. The combined ether filtrate was concentrated under reduced pressure inside the glove box. The residue was purified by vacuum distillation (bp. $80-90{ }^{\circ} \mathrm{C} / 0.4 \mathrm{mmHg}$ ) to give boron amide 6 as a colorless liquid $(2.53 \mathrm{~g}, 76 \%) .{ }^{1} \mathrm{H}$ NMR (THF- $\left.d_{8}, 600 \mathrm{MHz}\right) \delta$ : $3.74(\mathrm{~s}, 4 \mathrm{H}), 1.10(\mathrm{~s}, 6 \mathrm{H}), 0.31(\mathrm{~s}, 12 \mathrm{H}) ;{ }^{13} \mathrm{C}$ NMR $\left(\mathrm{THF}-d_{8}, 150 \mathrm{MHz}\right) \delta: 73.7,32.9$, 22.8, 4.3; ${ }^{11} \mathrm{~B}$ NMR (THF- $\left.d_{8}, 192 \mathrm{MHz}\right) \delta$ : 20.9; HRMS (DART) calcd. for $\mathrm{C}_{11} \mathrm{H}_{29} \mathrm{BNO}_{2} \mathrm{Si}_{2}{ }^{+}=[\mathrm{M}+\mathrm{H}]^{+}: m / z=274.1830$, found: $m / z=274.1836$. 


\title{
V. Analytical Data for Allenyl Alcohols and Homopropargyl
}

\author{
Alcohols \\ 2-Phenylpenta-3,4-dien-2-ol (3a) ${ }^{8}$ \\ $\mathrm{HO} \mathrm{Me}={ }^{1} \mathrm{H} \mathrm{NMR}\left(\mathrm{CDCl}_{3}, 600 \mathrm{MHz}\right) \delta 7.52-7.50(\mathrm{~m}, 2 \mathrm{H}), 7.36-7.34(\mathrm{~m}$, \\ X" 2H), 7.27-7.26(m,1H), 5.58-5.56(m,1H), 5.00-4.94 (m, 2H), 2.10 \\ 3a \\ $(\mathrm{s}, 1 \mathrm{H}), 1.66(\mathrm{~s}, 3 \mathrm{H}) ;{ }^{13} \mathrm{C} \mathrm{NMR}\left(\mathrm{CDCl}_{3}, 150 \mathrm{MHz}\right) \delta 205.8,147.1$, \\ 128.2, 127.0, 124.9, 100.2, 79.2, 73.0, 30.4.
}

2-(4-Methoxyphenyl)penta-3,4-dien-2-ol (3b)<smiles>C=C=CC(C)(O)c1ccc(OC)cc1</smiles>

${ }^{1} \mathrm{H}$ NMR $\left(\mathrm{CDCl}_{3}, 600 \mathrm{MHz}\right) \delta$ 7.43-7.40 (m, 2H), 6.89-6.87 $(\mathrm{m}, 2 \mathrm{H}), 5.55-5.53(\mathrm{~m}, 1 \mathrm{H}), 4.98-4.92(\mathrm{~m}, 2 \mathrm{H}), 3.80(\mathrm{~s}, 3 \mathrm{H})$, $2.14(\mathrm{~s}, 1 \mathrm{H}), 1.64(\mathrm{~s}, 3 \mathrm{H}) ;{ }^{13} \mathrm{C} \mathrm{NMR}\left(\mathrm{CDCl}_{3}, 150 \mathrm{MHz}\right) \delta$ 205.7, 158.5, 139.3, 126.2, 126.0, 113.5, 113.5, 100.3, 79.0, 72.7, 55.3, 30.4; IR (neat): $v=3297,2978,2933,2836,1956$, $1611,1583,1512,1462,1415 \mathrm{~cm}^{-1}$; HRMS (DART) calcd. for $\mathrm{C}_{12} \mathrm{H}_{13} \mathrm{O}^{+}[\mathrm{M}-\mathrm{OH}]^{+}$: 173.0966, found: 173.0966 .

\section{2-(4-Bromophenyl)penta-3,4-dien-2-ol (3c)}

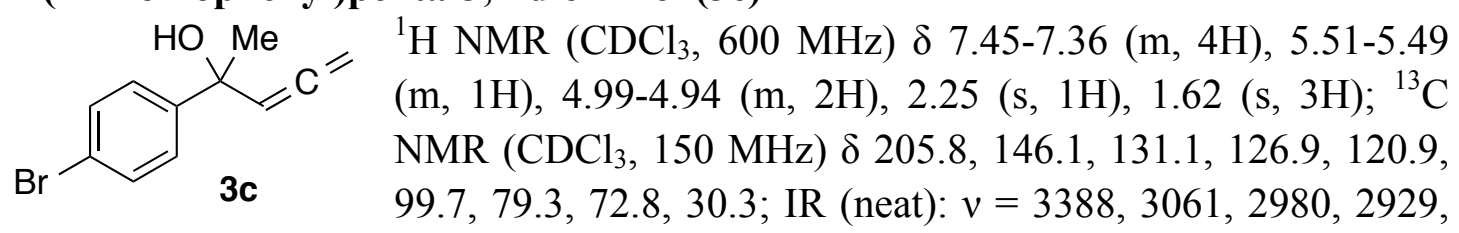
2868, 1956, 1906, 1675, 1591, 1486, $1448 \mathrm{~cm}^{-1}$; HRMS (DART) calcd. for $\mathrm{C}_{11} \mathrm{H}_{10} \mathrm{Br}^{+}$ $[\mathrm{M}-\mathrm{OH}]^{+}:$220.9966, found: 220.9963 .

\section{2-(4-Nitrophenyl)penta-3,4-dien-2-ol (3d)}

$$
\begin{aligned}
& \begin{array}{l}
1 \mathrm{H}), 1.68(\mathrm{~s}, 3 \mathrm{H}) ;{ }^{13} \mathrm{C} \mathrm{NMR}\left(\mathrm{CDCl}_{3}, 150 \mathrm{MHz}\right) \delta 206.0, \\
154.3,146.8,126.1,125.9,123.4,123.3,99.2,79.7,73.0,
\end{array}
\end{aligned}
$$
30.4; IR (neat): $v=3440,3301,3110,3075,2982,2932,2862,2453,1956,1801$, $1690,1602,1519,1449,1407 \mathrm{~cm}^{-1}$. HRMS (DART) calcd. for $\mathrm{C}_{11} \mathrm{H}_{10} \mathrm{NO}_{2}^{+}[\mathrm{M}-\mathrm{OH}]^{+}$: 188.0711, found: 188.0712 .

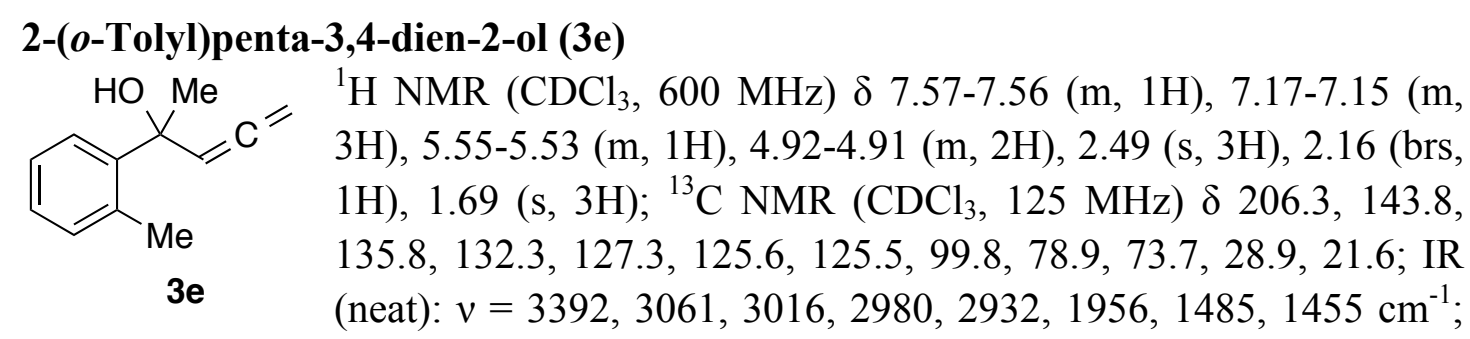

HRMS (DART) calcd. for $\mathrm{C}_{12} \mathrm{H}_{13}{ }^{+}[\mathrm{M}-\mathrm{OH}]^{+}:$157.1017, found: 157.1015. 


\section{2-(Thiophen-2-yl)penta-3,4-dien-2-ol (3f)}

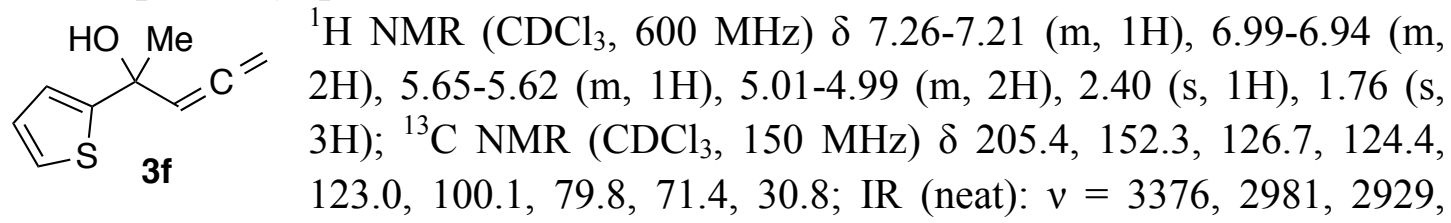

1956, $1433 \mathrm{~cm}^{-1}$; HRMS (DART) calcd. for $\mathrm{C}_{9} \mathrm{H}_{9} \mathrm{~S}^{+}[\mathrm{M}-\mathrm{OH}]^{+}:$149.0425, found: 149.0420 .

\section{2-(Naphthalen-1-yl)penta-3,4-dien-2-ol (3g)}<smiles>C=C=CC(C)(O)c1cccc2ccccc12</smiles>

$3 g$

${ }^{1} \mathrm{H}$ NMR $\left(\mathrm{CDCl}_{3}, 600 \mathrm{MHz}\right) \delta 8.57-8.55(\mathrm{~m}, 1 \mathrm{H}), 7.85-7.74(\mathrm{~m}$, $3 \mathrm{H})$, 7.49-7.40 (m, 3H), 5.79-5.77 (m, 1H), 4.96-4.90 (m, 2H), $2.31(\mathrm{~s}, 1 \mathrm{H}), 1.91(\mathrm{~s}, 3 \mathrm{H}) ;{ }^{13} \mathrm{C} \mathrm{NMR}\left(\mathrm{CDCl}_{3}, 150 \mathrm{MHz}\right) \delta 206.6$, $141.7,134.7,130.5,128.9,128.7,126.7,125.3,125.1,124.8$, 123.3, 100.7, 79.1, 74.2, 29.8; IR (neat): $v=3551,3400,3049$, 2981, 2933, 1955, 1709, 1598, 1509, $1449 \mathrm{~cm}^{-1}$; HRMS (DART) calcd. for $\mathrm{C}_{15} \mathrm{H}_{13}{ }^{+}$ $[\mathrm{M}-\mathrm{OH}]^{+}: 193.1017$, found: 193.1013.<smiles>C=C=CC(C)(O)CCc1ccccc1</smiles>

$3 \mathrm{~h}$

$44.6,30.6,28.2$.

\section{4-Methylocta-1,2-dien-4-ol (3i)}

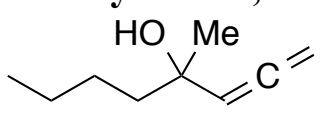

3i

${ }^{1} \mathrm{H} \mathrm{NMR}\left(\mathrm{CDCl}_{3}, 600 \mathrm{MHz}\right)$ ઈ 5.28-5.26 (m, 1H), 4.89-4.87 (m, $2 \mathrm{H}), 1.59-1.56(\mathrm{~m}, 3 \mathrm{H}), 1.33-1.31(\mathrm{~m}, 7 \mathrm{H}), 0.92-0.90(\mathrm{~m}, 3 \mathrm{H})$; ${ }^{13} \mathrm{C} \mathrm{NMR}\left(\mathrm{CDCl}_{3}, 150 \mathrm{MHz}\right) \delta 99.4,78.3,71.3,42.6,27.8$, 26.3, 23.0, 15.6, 14.0; IR (neat): $v=3363,2958,2934,2864,1957,1461 \mathrm{~cm}^{-1}$; HRMS (DART) calcd. for $\mathrm{C}_{9} \mathrm{H}_{15}{ }^{+}[\mathrm{M}-\mathrm{OH}]^{+}:$: 123.1174, found: 123.1168 .

\section{1-(Propa-1,2-dien-1-yl)cyclohexanol (3j) ${ }^{9}$}

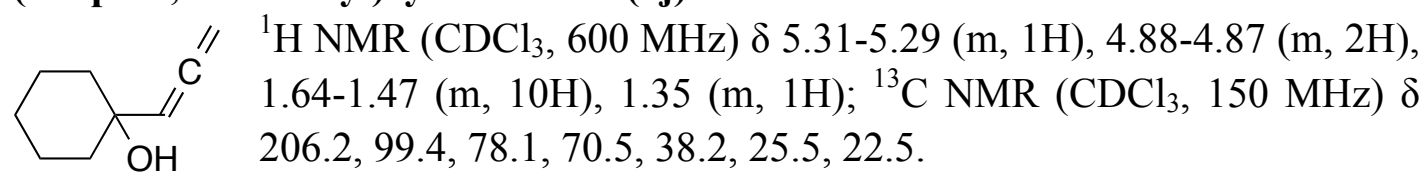

3j

\section{3-phenylhexa-4,5-dien-3-ol (3k) ${ }^{10}$}<smiles>C=C=CC(O)(CC)c1ccccc1</smiles>

3k

${ }^{1} \mathrm{H}$ NMR $\left(\mathrm{CDCl}_{3}, 600 \mathrm{MHz}\right) \delta 7.50-7.40(\mathrm{~m}, 2 \mathrm{H}), 7.38-7.29(\mathrm{~m}$, 2H), $7.24(\mathrm{dt}, J=8.0,4.3 \mathrm{~Hz}, 1 \mathrm{H}), 5.57(\mathrm{t}, J=6.7 \mathrm{~Hz}, 1 \mathrm{H}), 5.03-$ $4.85(\mathrm{~m}, 2 \mathrm{H}), 2.08(\mathrm{~s}, 1 \mathrm{H}), 2.00-1.83(\mathrm{~m}, 2 \mathrm{H}), 0.84(\mathrm{t}, J=7.4 \mathrm{~Hz}$, $3 \mathrm{H}) .{ }^{13} \mathrm{C} \mathrm{NMR}\left(\mathrm{CDCl}_{3}, 151 \mathrm{MHz}\right) \delta 205.9,145.9,128.1,126.9$, $125.5,99.6,79.4,35.6,8.2$. 


\section{6-methyl-4-phenylhepta-1,2-dien-4-ol (3I)}

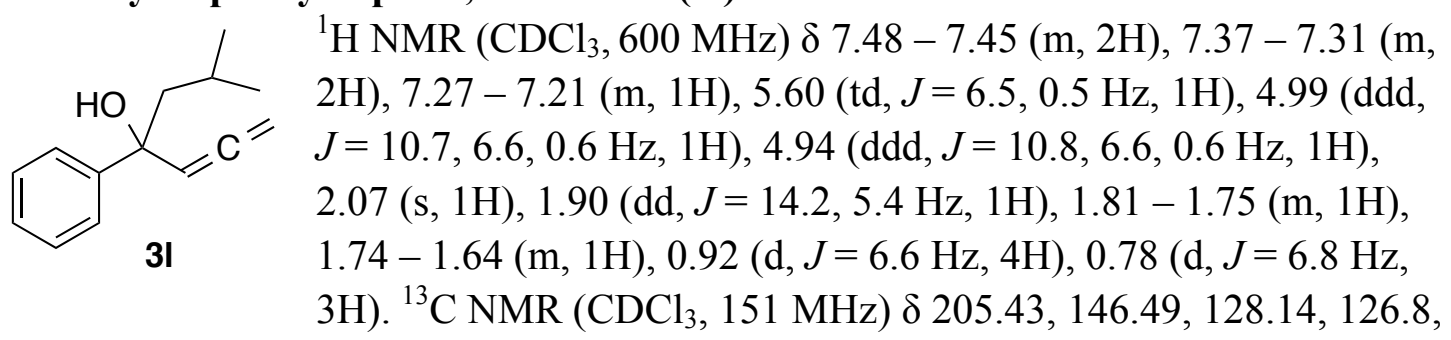

$125.3,100.8,79.8,75.4,51.5,24.5,24.5,24.4$. IR (neat): $v=3563,3465,2953,1955$, $1601,1493 \mathrm{~cm}^{-1}$; HRMS (DART) calcd. for $\mathrm{C}_{14} \mathrm{H}_{17}{ }^{+}[\mathrm{M}-\mathrm{OH}]^{+}: 185.1330$, found: 185.1331 .

\section{1,1-Diphenylbuta-2,3-dien-1-ol (3m)}<smiles>C=C=CC(O)(c1ccccc1)c1ccccc1</smiles>

$3 m$

${ }^{1} \mathrm{H}$ NMR $\left(\mathrm{CDCl}_{3}, 600 \mathrm{MHz}\right) \delta$ 7.44-7.24 (m, 10H), 5.95-5.93 (m, $1 \mathrm{H}), 4.97-4.96(\mathrm{~m}, 2 \mathrm{H}), 2.65(\mathrm{~s}, 1 \mathrm{H}) ;{ }^{13} \mathrm{C} \mathrm{NMR}\left(\mathrm{CDCl}_{3}, 150 \mathrm{MHz}\right)$ $\delta$ 205.6, 146.0, 128.1, 127.3, 126.6, 100.1, 80.3, 77.0; IR (neat): $v$ $=3551,3453,3299,3060,3028,1956,1600,1492,1447 \mathrm{~cm}^{-1}$; HRMS (DART) calcd. for $\mathrm{C}_{16} \mathrm{H}_{13}{ }^{+}[\mathrm{M}-\mathrm{OH}]^{+}:$205.1017, found: 205.1020 .<smiles>C=C=CC(O)(c1ccccc1)C(C)C</smiles>

\section{y-2-phenylpenta-3,4-dienoate (3n)}

${ }^{1} \mathrm{H}$ NMR $\left(\mathrm{CDCl}_{3}, 600 \mathrm{MHz}\right) \delta 7.59-7.58(\mathrm{~m}, 2 \mathrm{H}), 7.38-7.31(\mathrm{~m}$, $3 \mathrm{H}), 5.77-5.75(\mathrm{~m}, 1 \mathrm{H}), 5.01-4.99(\mathrm{~m}, 2 \mathrm{H}), 3.91(\mathrm{~s}, 1 \mathrm{H}), 3.77(\mathrm{~s}$, $3 \mathrm{H}) ;{ }^{13} \mathrm{C} \mathrm{NMR}\left(\mathrm{CDCl}_{3}, 150 \mathrm{MHz}\right) \delta 206.9,174.0,140.5,128.3$, 128.2, 126.2, 95.3, 79.6, 76.9, 53.3; IR (neat): $v=3495,3062$, 3029, 2953, 1958, 1732, 1493, 1448, $1437 \mathrm{~cm}^{-1}$; HRMS (DART) calcd. for $\mathrm{C}_{12} \mathrm{H}_{11} \mathrm{O}_{2}{ }^{+}[\mathrm{M}-\mathrm{OH}]^{+}:$187.0759, found: $187.0758 ;[\alpha]^{20}{ }_{\mathrm{D}}=-45.0^{\circ}$ (c 0.33, $\mathrm{CHCl}_{3}, 68 \%$ ee); HPLC conditions: Chiralpak OJ-H column [hexane/i-PrOH (91:9) $0.5 \mathrm{~mL} / \mathrm{min}$ ], $t_{\mathrm{R}}=42.8 \mathrm{~min}$ (minor), $t_{\mathrm{R}}=50.1 \mathrm{~min}$ (major).

\section{1-(p-Tolyl)buta-2,3-dien-1-ol (3o) ${ }^{11}$}<smiles>C=C=CC(O)c1ccc(C)cc1</smiles>

30 ${ }^{1} \mathrm{H}$ NMR $\left(\mathrm{CDCl}_{3}, 600 \mathrm{MHz}\right) \delta$ 7.28-7.13 (m, 4H), 5.42-5.41 $(\mathrm{m}, 1 \mathrm{H}), 5.22(\mathrm{~s}, 1 \mathrm{H}), 4.91(\mathrm{~m}, 2 \mathrm{H}), 2.34(\mathrm{~s}, 3 \mathrm{H}), 2.23(\mathrm{~s}, 1 \mathrm{H})$; ${ }^{13} \mathrm{C} \mathrm{NMR}\left(\mathrm{CDCl}_{3}, 150 \mathrm{MHz}\right) \delta 207.0,139.9,137.4,129.1$, 126.0, 95.2, 78.0, 71.7, 21.1.

\section{1-(4-methoxyphenyl)buta-2,3-dien-1-ol (3p) ${ }^{3}$}

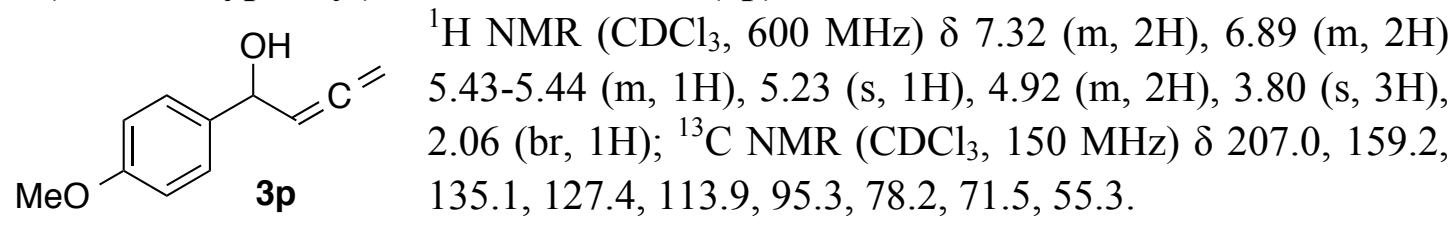




\section{1-(4-Nitrophenyl)buta-2,3-dien-1-ol (3q) ${ }^{12}$}<smiles>C=C=CC(O)c1ccc([N+](=O)[O-])cc1</smiles>

${ }^{1} \mathrm{H}$ NMR $\left(\mathrm{CDCl}_{3}, 600 \mathrm{MHz}\right) \delta 8.22-8.21(\mathrm{~m}, 2 \mathrm{H}), 7.58-7.57$ $(\mathrm{m}, 2 \mathrm{H}), 5.42-5.38(\mathrm{~m}, 2 \mathrm{H}), 4.97-4.96(\mathrm{~m}, 2 \mathrm{H}), 2.32(\mathrm{~s}, 1 \mathrm{H})$; ${ }^{13} \mathrm{C} \mathrm{NMR}\left(\mathrm{CDCl}_{3}, 150 \mathrm{MHz}\right) \delta 207.4,149.8,147.4,126.8$, 123.7, 94.5, 78.9, 71.2.

\section{1-(Naphthalen-2-yl)buta-2,3-dien-1-ol (3r) ${ }^{11}$}<smiles>C=C=CC(O)c1ccc2ccccc2c1</smiles>

${ }^{1} \mathrm{H} \mathrm{NMR}\left(\mathrm{CDCl}_{3}, 600 \mathrm{MHz}\right) \delta$ 7.83-7.82 (m, 4H), 7.50-7.46 $(\mathrm{m}, 3 \mathrm{H}), 5.51-5.50(\mathrm{~m}, 1 \mathrm{H}), 5.42(\mathrm{~m}, 1 \mathrm{H}), 4.94(\mathrm{~m}, 2 \mathrm{H}), 2.33$ $(\mathrm{s}, 1 \mathrm{H}) ;{ }^{13} \mathrm{C} \mathrm{NMR}\left(\mathrm{CDCl}_{3}, 150 \mathrm{MHz}\right) \delta 207.2,140.2,133.2$, $133.0,128.3,128.0,127.7,126.1,125.9,124.6,124.3,95.1$, $78.3,72.0$.

\section{1-(Furan-2-yl)buta-2,3-dien-1-ol (3s) ${ }^{13}$}<smiles>C=C=CC(O)c1ccco1</smiles>

3s ${ }^{1} \mathrm{H}$ NMR $\left(\mathrm{CDCl}_{3}, 600 \mathrm{MHz}\right) \delta 7.40(\mathrm{~m}, 1 \mathrm{H}), 6.34-6.30(\mathrm{~m}, 2 \mathrm{H})$, 5.54-5.51 (m, 1H), $5.26(\mathrm{~m}, 1 \mathrm{H}), 4.99-4.94(\mathrm{~m}, 2 \mathrm{H}), 2.36(\mathrm{~s}, 1 \mathrm{H})$; ${ }^{13} \mathrm{C} \mathrm{NMR}\left(\mathrm{CDCl}_{3}, 150 \mathrm{MHz}\right) \delta 207.6,154.9,142.4,110.2,106.6$, 92.1, 78.6, 65.6.

\section{1-Phenylhexa-4,5-dien-3-ol (3t) ${ }^{14}$}<smiles>C=C=CC(O)CCc1ccccc1</smiles>

3t

${ }^{1} \mathrm{H}$ NMR $\left(\mathrm{CDCl}_{3}, 600 \mathrm{MHz}\right) \delta$ 7.29-7.18 (m, 5H), 5.29-5.26 $(\mathrm{m}, 1 \mathrm{H}), 4.88-4.87(\mathrm{~m}, 2 \mathrm{H}), 4.20(\mathrm{~s}, 1 \mathrm{H}), 2.80-2.69(\mathrm{~m}, 2 \mathrm{H})$, 1.91-1.88 (m, 2H), $1.76(\mathrm{~s}, 1 \mathrm{H}) ;{ }^{13} \mathrm{C} \mathrm{NMR}\left(\mathrm{CDCl}_{3}, 150 \mathrm{MHz}\right)$ $\delta 207.0,141.8,128.4,128.4,125.8,94.7,77.7,68.9,39.0$,

31.7 .

\section{2-phenylpent-4-yn-2-ol (4a) ${ }^{15}$}<smiles>C#CCC(C)(O)c1ccccc1</smiles>

${ }^{1} \mathrm{H} \mathrm{NMR}\left(\mathrm{CDCl}_{3}, 600 \mathrm{MHz}\right) \delta 7.48(\mathrm{~d}, J=1.6 \mathrm{~Hz}, 2 \mathrm{H}), 7.36(\mathrm{~d}, J=$ $6.6 \mathrm{~Hz}, 2 \mathrm{H}), 7.27(\mathrm{~s}, 1 \mathrm{H}), 2.76(\mathrm{~d}, J=16.6 \mathrm{~Hz}, 1 \mathrm{H}), 2.68(\mathrm{~d}, J=$ $16.6 \mathrm{~Hz}, 1 \mathrm{H}), 2.46(\mathrm{~s}, 1 \mathrm{H}), 2.05(\mathrm{~s}, 1 \mathrm{H}), 1.64(\mathrm{~d}, J=9.5 \mathrm{~Hz}, 3 \mathrm{H})$. ${ }^{13} \mathrm{C}$ NMR $\left(151 \mathrm{MHz}, \mathrm{CDCl}_{3}\right) \delta 146.4,128.4,127.2,124.8,80.5$, $73.3,71.9,34.7,29.3$.

\section{2-(4-methoxyphenyl)pent-4-yn-2-ol (4b) ${ }^{10}$}<smiles>C#CCC(C)(O)c1ccc(OC)cc1</smiles>
${ }^{1} \mathrm{H} \mathrm{NMR}\left(\mathrm{CDCl}_{3}, 600 \mathrm{MHz}\right) \delta 7.41(\mathrm{~d}, J=8.4 \mathrm{~Hz}, 2 \mathrm{H}), 6.89$ $(\mathrm{d}, J=8.4 \mathrm{~Hz}, 2 \mathrm{H}), 3.81(\mathrm{~s}, 3 \mathrm{H}), 2.75(\mathrm{~d}, J=16.6 \mathrm{~Hz}, 1 \mathrm{H})$, $2.67(\mathrm{~d}, J=16.6 \mathrm{~Hz}, 1 \mathrm{H}), 2.35(\mathrm{~s}, 1 \mathrm{H}), 2.05(\mathrm{~s}, 1 \mathrm{H}), 1.63(\mathrm{~s}$, $3 \mathrm{H}) .{ }^{13} \mathrm{C} \mathrm{NMR}\left(\mathrm{CDCl}_{3}, 151 \mathrm{MHz}\right) \delta 158.7,138.6,126.0$, $113.6,80.7,73.0,71.7,55.3,34.7,29.3$.

\section{2-(4-bromophenyl)pent-4-yn-2-ol (4c) ${ }^{10}$}

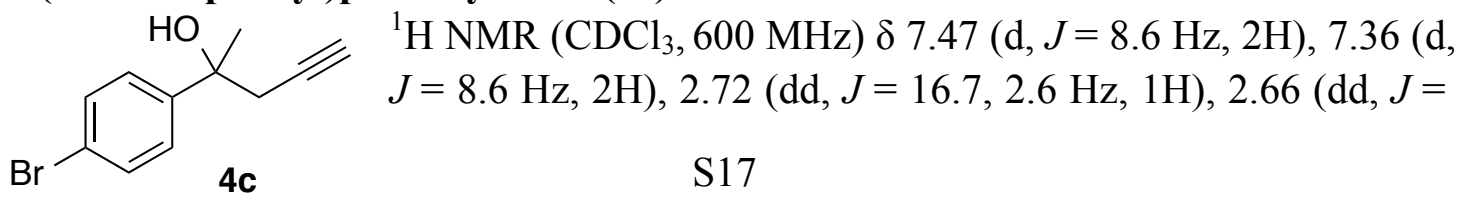


16.7, $2.6 \mathrm{~Hz}, 1 \mathrm{H}), 2.42(\mathrm{~s}, 1 \mathrm{H}), 2.06(\mathrm{t}, J=2.6 \mathrm{~Hz}, 1 \mathrm{H}), 1.61(\mathrm{~s}, 3 \mathrm{H}) .{ }^{13} \mathrm{C} \mathrm{NMR}$ $\left(\mathrm{CDCl}_{3}, 151 \mathrm{MHz}\right) \delta 153.7,147.1,126.04,123.5,79.5,73.3,72.6,34.5,29.2$; ee: $90 \%,[\alpha]_{\mathrm{D}}{ }^{32}=-45.4^{\circ}\left(\mathrm{c}=0.1, \mathrm{CHCl}_{3}\right)$; HPLC conditions: Chiralpak AD-H column [hexane $/ i-\mathrm{PrOH}(99: 1) 1.0 \mathrm{~mL} / \mathrm{min}], t_{\mathrm{R}}=35.1 \mathrm{~min}$ (minor), $t_{\mathrm{R}}=38.9 \mathrm{~min}$ (major)

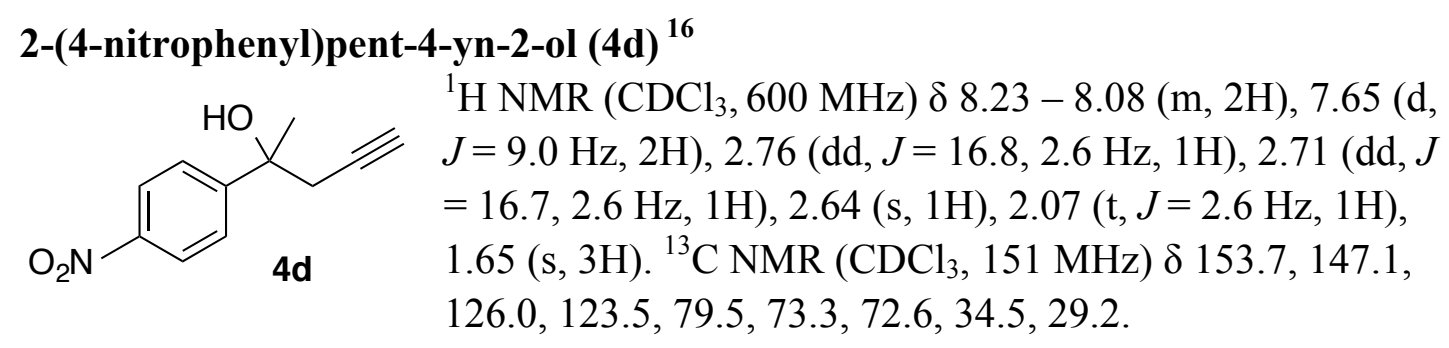

\section{2-(naphthalen-1-yl)pent-4-yn-2-ol (4g) ${ }^{16}$}

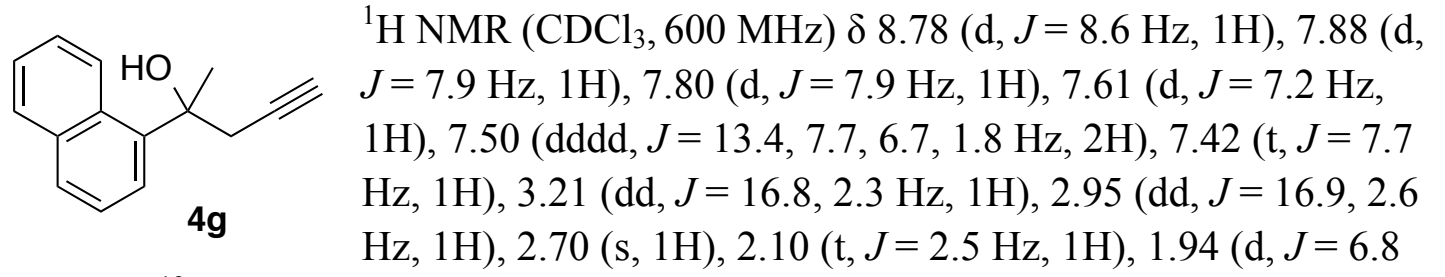

$\mathrm{Hz}, 3 \mathrm{H}) .{ }^{13} \mathrm{C} \mathrm{NMR}\left(\mathrm{CDCl}_{3}, 151 \mathrm{MHz}\right) \delta 140.6,135.1,131.0,129.4,129.2,126.9$, 125.7, 125.4, 124.8, 123.9, 80.8, 75.0, 72.3, 33.9, 29.3. HRMS (ESI) calcd. for: $\mathrm{C}_{15} \mathrm{H}_{13}{ }^{+}[\mathrm{M}-\mathrm{OH}]^{+}: 193.1017$, found: 193.1021 .

\section{3-methyl-1-phenylhex-5-yn-3-ol (4h) ${ }^{18}$}

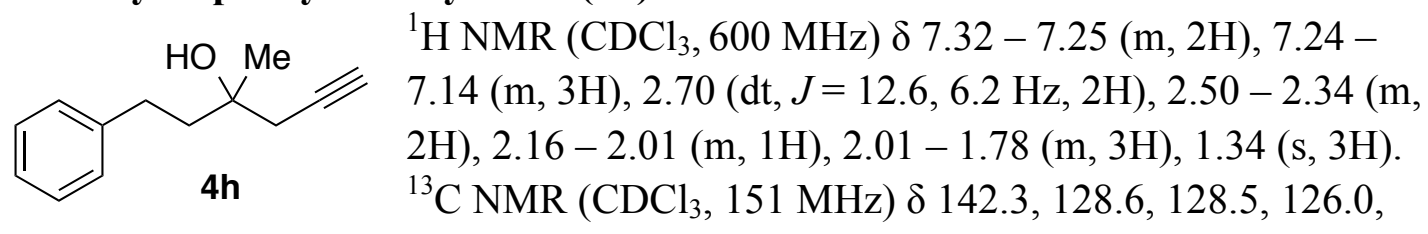

80.7, 71.6, 71.5, 43.1, 32.6, 30.4, 26.5. 


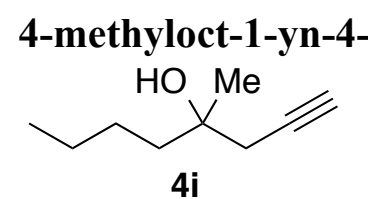

19 19

${ }^{1} \mathrm{H}$ NMR $\left(\mathrm{CDCl}_{3}, 600 \mathrm{MHz}\right) \delta 2.40-2.28(\mathrm{~m}, 2 \mathrm{H}), 2.05(\mathrm{dd}, J=$ 4.4, 1.6 Hz, 1H), $1.80(\mathrm{~s}, 1 \mathrm{H}), 1.61-1.49(\mathrm{~m}, 2 \mathrm{H}), 1.31$ (ddd, $J$ $=13.2,8.0,4.2 \mathrm{~Hz}, 4 \mathrm{H}), 1.24(\mathrm{~d}, J=3.0 \mathrm{~Hz}, 3 \mathrm{H}), 0.90(\mathrm{td}, J=$ 6.6, $2.7 \mathrm{~Hz}, 3 \mathrm{H}) .{ }^{13} \mathrm{C} \mathrm{NMR}\left(\mathrm{CDCl}_{3}, 151 \mathrm{MHz}\right) \delta 81.1,71.7,71.3,41.0,32.4,26.4$, $26.2,23.2,14.1$.

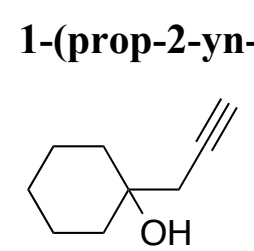

-yl)cyclohexanol $(4 \mathrm{j})^{20}$

${ }^{1} \mathrm{H} \mathrm{NMR}\left(\mathrm{CDCl}_{3}, 600 \mathrm{MHz}\right) \delta 2.36(\mathrm{dd}, J=2.7,1.4 \mathrm{~Hz}, 2 \mathrm{H}), 2.12-$ $2.06(\mathrm{~m}, 1 \mathrm{H}), 1.79(\mathrm{~s}, 1 \mathrm{H}), 1.72-1.58(\mathrm{~m}, 5 \mathrm{H}), 1.58-1.43(\mathrm{~m}, 5 \mathrm{H})$.

${ }^{13} \mathrm{C} \mathrm{NMR}\left(\mathrm{CDCl}_{3}, 151 \mathrm{MHz}\right) \delta 80.7,78.2,71.5,70.4,36.8,25.7,22.2$.

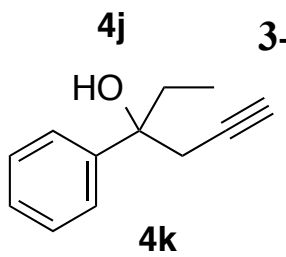

3-phenylhex-5-yn-3-ol (4k) ${ }^{10}$

${ }^{1} \mathrm{H}$ NMR $\left(\mathrm{CDCl}_{3}, 600 \mathrm{MHz}\right) \delta 7.38-7.33(\mathrm{~m}, 2 \mathrm{H}), 7.31-7.25(\mathrm{~m}$, $2 \mathrm{H}), 7.22-7.15(\mathrm{~m}, 1 \mathrm{H}), 2.81-2.54(\mathrm{~m}, 2 \mathrm{H}), 2.24(\mathrm{~s}, 1 \mathrm{H}), 2.00-$ $1.74(\mathrm{~m}, 3 \mathrm{H}), 0.70(\mathrm{t}, J=7.5 \mathrm{~Hz}, 3 \mathrm{H}) .{ }^{13} \mathrm{C} \mathrm{NMR}\left(\mathrm{CDCl}_{3}, 151 \mathrm{MHz}\right)$ $\delta 144.7,128.2,127.0,125.4,80.4,75.7,71.9,34.4,33.2,8.0$.

\section{6-methyl-4-phenylhept-1-yn-4-ol (4I)}<smiles>C#CCC(O)(CC(C)C)c1ccccc1</smiles>

41

${ }^{1} \mathrm{H} \mathrm{NMR}\left(\mathrm{CDCl}_{3}, 600 \mathrm{MHz}\right) \delta 7.43(\mathrm{~d}, \mathrm{~J}=7.7 \mathrm{~Hz}, 2 \mathrm{H}), 7.34(\mathrm{t}, J=$

$7.7 \mathrm{~Hz}, 2 \mathrm{H}), 7.25(\mathrm{dt}, J=8.0,1.1 \mathrm{~Hz}, 1 \mathrm{H}), 2.78-2.64(\mathrm{~m}, 2 \mathrm{H})$, $2.35(\mathrm{~s}, 1 \mathrm{H}), 2.04-1.99(\mathrm{~m}, 1 \mathrm{H}), 1.93-1.74(\mathrm{~m}, 2 \mathrm{H}), 1.63-1.50$ $(\mathrm{m}, 1 \mathrm{H}), 0.92-0.86(\mathrm{~m}, 3 \mathrm{H}), 0.73-0.66(\mathrm{~m}, 3 \mathrm{H}) .{ }^{13} \mathrm{C} \mathrm{NMR}$ $\left(\mathrm{CDCl}_{3}, 151 \mathrm{MHz}\right) \delta 145.2,128.2,128.1,126.9,125.3,125.3,80.4$, $75.9,72.0,50.2,34.9,24.5,24.3,24.2$; IR (neat): $v=3555,3481$, 3304, 3088, 3061, 3029, 2954, 2927, 2870, 2118, 1955, 1603, 1495, 1467, 1447 $\mathrm{cm}^{-1}$;HRMS (ESI) calcd. for: $\mathrm{C}_{14} \mathrm{H}_{17}{ }^{+}[\mathrm{M}-\mathrm{OH}]^{+}: 185.1330$, found: 185.1329 .

\section{1,1-diphenylbut-3-yn-1-ol (4m) ${ }^{21}$}<smiles>C#CCC(O)(c1ccccc1)c1ccccc1</smiles>

${ }^{1} \mathrm{H}$ NMR $\left(\mathrm{CDCl}_{3}, 600 \mathrm{MHz}\right) \delta 7.47-7.41(\mathrm{~m}, 4 \mathrm{H}), 7.32(\mathrm{t}, J=7.5$ $\mathrm{Hz}, 4 \mathrm{H}), 7.28-7.23(\mathrm{~m}, 2 \mathrm{H}), 3.16(\mathrm{~d}, J=2.3 \mathrm{~Hz}, 2 \mathrm{H}), 2.95(\mathrm{~s}, 1 \mathrm{H})$, $2.04(\mathrm{~s}, 1 \mathrm{H}) .{ }^{13} \mathrm{C} \mathrm{NMR}\left(\mathrm{CDCl}_{3}, 151 \mathrm{MHz}\right) \delta 146.1,145.5,128.3$, $127.4,126.7,126.2,100.2,80.4,72.6,33.5$.

\section{methyl 2-hydroxy-2-phenylpent-4-ynoate (4n)}

$$
\begin{aligned}
& 3.9 \mathrm{~Hz}, 2 \mathrm{H}), 7.31(\mathrm{t}, J=7.3 \mathrm{~Hz}, 1 \mathrm{H}), 3.94(\mathrm{~s}, 1 \mathrm{H}), 3.81(\mathrm{~s}, 3 \mathrm{H}), \\
& 4.17(\mathrm{dd}, J=16.6,2.6 \mathrm{~Hz}, 1 \mathrm{H}), 2.84(\mathrm{dd}, J=16.6,2.6 \mathrm{~Hz}, 1 \mathrm{H}), \\
& 2.05(\mathrm{t}, J=2.6 \mathrm{~Hz}, 1 \mathrm{H}) .{ }^{13} \mathrm{C} \mathrm{NMR}\left(\mathrm{CDCl}_{3}, 151 \mathrm{MHz}\right) \delta 174.0,
\end{aligned}
$$
$140.0,128.5,128.4,125.5,79.1,77.6,71.3,53.6,31.0$.

\section{1-(p-tolyl)but-3-yn-1-ol (4o) ${ }^{22}$}<smiles>C#CCC(O)c1ccc(C)cc1</smiles>

${ }^{1} \mathrm{H} \mathrm{NMR}\left(\mathrm{CDCl}_{3}, 600 \mathrm{MHz}\right) \delta 7.26(\mathrm{~d}, J=8.0 \mathrm{~Hz}, 2 \mathrm{H}), 7.16$ $(\mathrm{d}, J=7.9 \mathrm{~Hz}, 2 \mathrm{H}), 4.82(\mathrm{t}, J=6.4 \mathrm{~Hz}, 1 \mathrm{H}), 2.61(\mathrm{dd}, J=6.4$, 
$2.6 \mathrm{~Hz}, 2 \mathrm{H}), 2.41(\mathrm{~s}, 1 \mathrm{H}), 2.34(\mathrm{~s}, 3 \mathrm{H}), 2.05(\mathrm{t}, J=2.6 \mathrm{~Hz}, 1 \mathrm{H}) .{ }^{13} \mathrm{C} \mathrm{NMR}\left(\mathrm{CDCl}_{3}\right.$, $151 \mathrm{MHz}) \delta 139.7,137.8,129.3,125.8,80.9,72.3,71.0,29.4,21.2$.

\section{1-(4-methoxyphenyl)but-3-yn-1-ol (4p) ${ }^{3}$}

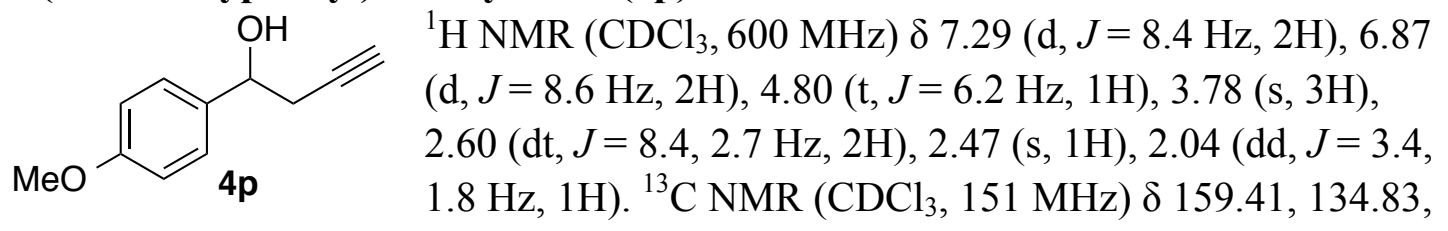
$127.14,113.95,80.96,72.06,70.93,55.35,29.41$.

\section{1-(4-nitrophenyl)but-3-yn-1-ol (4q)}<smiles>C#CCC(O)c1ccc([N+](=O)[O-])cc1</smiles>

${ }^{1} \mathrm{H} \mathrm{NMR}\left(\mathrm{CDCl}_{3}, 500 \mathrm{MHz}\right) \delta 8.22(\mathrm{~m}, 2 \mathrm{H}), 7.59(\mathrm{dm}, 2 \mathrm{H})$, $5.00(\mathrm{t}, J=5.8 \mathrm{~Hz}, 1 \mathrm{H}), 2.73-2.68(\mathrm{~m}, 2 \mathrm{H}), 2.68-2.61(\mathrm{~m}$, 2H), $2.11(\mathrm{br}, 1 \mathrm{H}) .{ }^{13} \mathrm{C} \mathrm{NMR}\left(\mathrm{CDCl}_{3}, 124 \mathrm{MHz}\right) \delta 149.4$, $147.5,126.6,123.6,79.3,71.9,71.2,29.5$. IR $(\mathrm{KBr}): v=$ 3532, 3250, 1954, 1605, $1516 \mathrm{~cm}^{-1}$; HRMS (DART) calcd. for $\mathrm{C}_{10} \mathrm{H}_{10} \mathrm{O}_{3} \mathrm{~N}^{+}[\mathrm{M}+\mathrm{H}]^{+}$: 192.0661 , found: 192.0655 .

\section{1-(naphthalen-2-yl)but-3-yn-1-ol (4r) ${ }^{22}$}

${ }^{1} \mathrm{H} \mathrm{NMR}\left(\mathrm{CDCl}_{3}, 600 \mathrm{MHz}\right) \delta 7.94-7.74(\mathrm{~m}, 4 \mathrm{H}), 7.57-7.38(\mathrm{~m}, 3 \mathrm{H}), 5.02(\mathrm{t}, J=$<smiles>C#CCC(O)c1ccc2ccccc2c1</smiles>
$6.3 \mathrm{~Hz}, 1 \mathrm{H}), 2.73(\mathrm{dd}, J=6.3,2.6 \mathrm{~Hz}, 2 \mathrm{H}), 2.70-2.59(\mathrm{~m}$, $1 \mathrm{H}), 2.08(\mathrm{dd}, J=3.3,2.0 \mathrm{~Hz}, 1 \mathrm{H}) .{ }^{13} \mathrm{C} \mathrm{NMR}\left(\mathrm{CDCl}_{3}, 151\right.$ $\mathrm{MHz}) \delta 140.0,133.3,128.4,128.2,127.8,127.8,126.3,126.2$, $124.8,123.8,80.8,72.6,71.2,29.5$.

\section{1-(furan-2-yl)but-3-yn-1-ol (4s)}<smiles>C#CCC(O)c1ccco1</smiles>

${ }^{1} \mathrm{NMR}\left(\mathrm{CDCl}_{3}, 500 \mathrm{MHz}\right) \delta 7.39(\mathrm{br}, 1 \mathrm{H}), 6.35(\mathrm{~m}, 2 \mathrm{H}), 4.88(\mathrm{br}$, $1 \mathrm{H}), 2.78(\mathrm{~m}, 2 \mathrm{H}), 2.50(\mathrm{br}, 1 \mathrm{H}), 2.08(\mathrm{~m}, 1 \mathrm{H}) .{ }^{13} \mathrm{C} \mathrm{NMR}\left(\mathrm{CDCl}_{3}\right.$, $151 \mathrm{MHz}) \delta 154.6,142.3,110.3,106.7,79.9,71.2,66.1,26.1 . \mathrm{IR}$ (neat): $v=3293,2919,2121,1957,1727,1680,1613,1503,1425$ $\mathrm{cm}^{-1}$; HRMS (DART) calcd. for $\mathrm{C}_{8} \mathrm{H}_{7} \mathrm{O}^{+}[\mathrm{M}-\mathrm{OH}]^{+}$: 119.0497,

found: 119.0499 .

\section{1-phenylhex-5-yn-3-ol (4t) ${ }^{22}$}<smiles>C#CCC(O)CCc1ccccc1</smiles>

${ }^{1} \mathrm{H}$ NMR $\left(\mathrm{CDCl}_{3}, 600 \mathrm{MHz}\right) \delta 7.31-7.26(\mathrm{~m}, 2 \mathrm{H}), 7.23-$ $7.17(\mathrm{~m}, 3 \mathrm{H}), 3.78(\mathrm{dq}, J=6.5,5.1 \mathrm{~Hz}, 1 \mathrm{H}), 2.88-2.74(\mathrm{~m}$, $1 \mathrm{H}), 2.70(\mathrm{dt}, J=13.8,8.1 \mathrm{~Hz}, 1 \mathrm{H}), 2.44$ (ddd, $J=16.7,4.8$, $2.7 \mathrm{~Hz}, 1 \mathrm{H}), 2.35$ (ddd, $J=16.8,6.7,2.7 \mathrm{~Hz}, 1 \mathrm{H}), 2.06$ (t, $J=$ $2.6 \mathrm{~Hz}, 1 \mathrm{H}), 1.91-1.79(\mathrm{~m}, 2 \mathrm{H}) .{ }^{13} \mathrm{C} \mathrm{NMR}\left(\mathrm{CDCl}_{3}, 151 \mathrm{MHz}\right) \delta 141.8,128.6,128.5$, $128.5,126.0,80.8,71.1,69.2,37.9,32.0,27.6$. 


\section{DFT Calculation}

All calculations were conducted with the Guassian 09 at DFT B3LYP level of theory with the LanL2DZ basis set. ${ }^{23}$ All optimized conformers were subjected to a freq check to verify the optimized structure is a stable structure. Geometries were optimized in the toluene solvent.
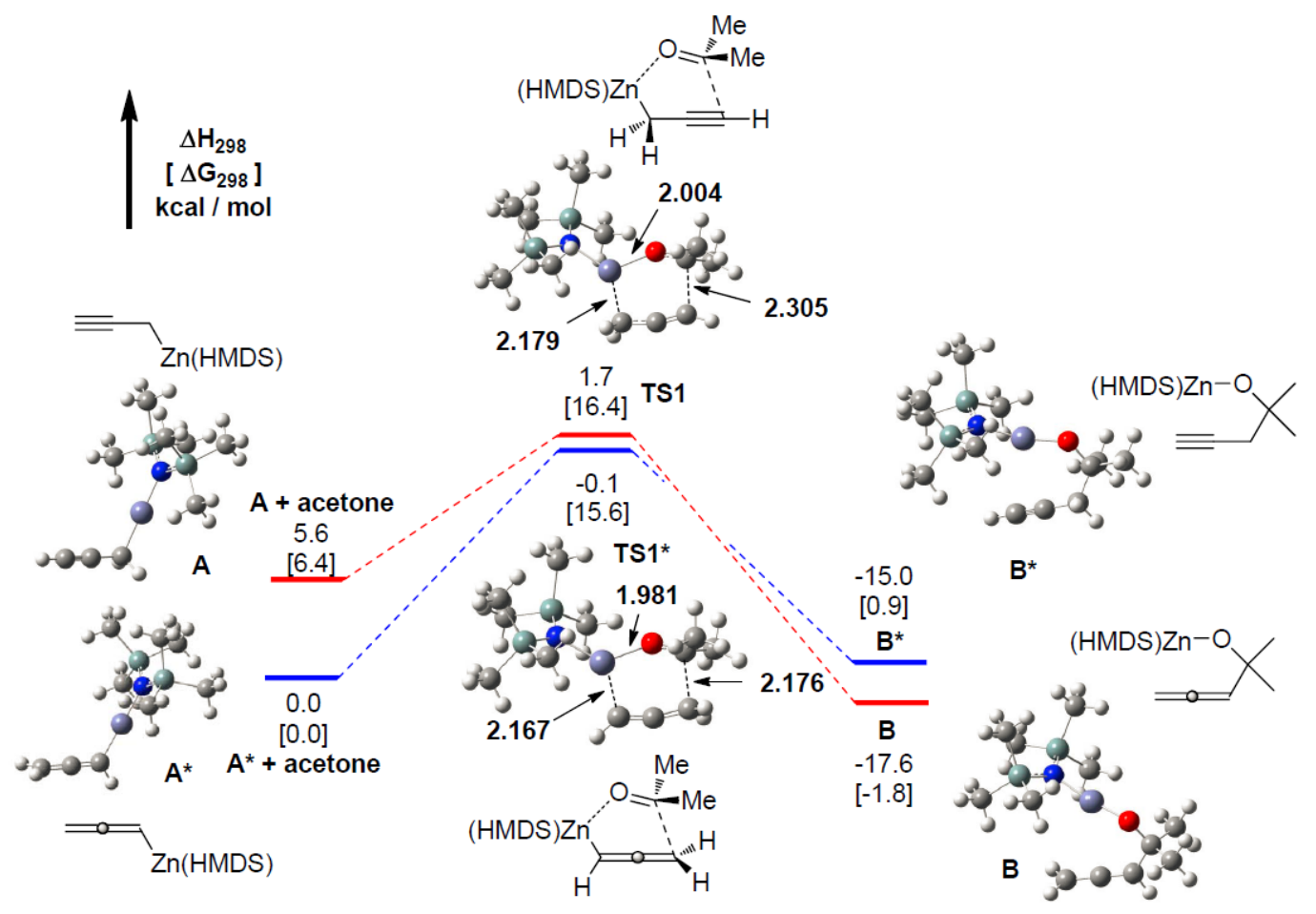

\section{Coordinates of All Stationary Points}

Acetone

\section{Standard orientation:}

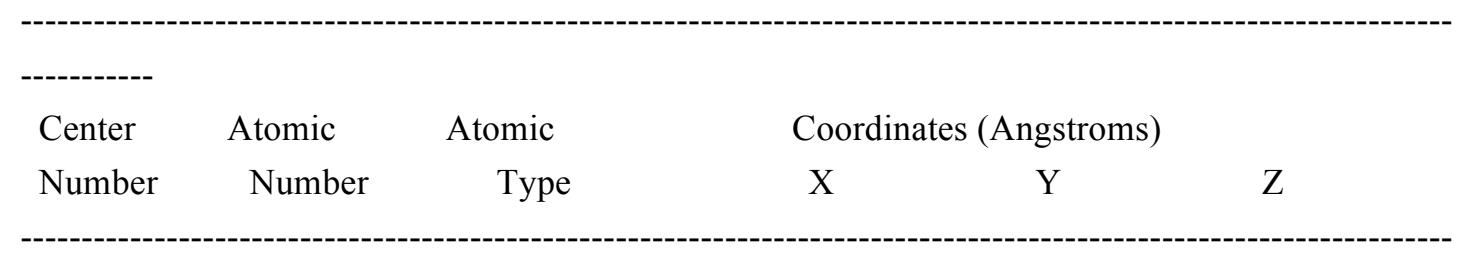

$\begin{array}{rrrrrc}1 & 6 & 0 & -0.000029 & 0.173622 & -0.000145 \\ 2 & 6 & 0 & -1.296013 & -0.621964 & 0.002555 \\ 3 & 1 & 0 & -1.416427 & -1.133577 & -0.962879 \\ 4 & 1 & 0 & -1.273543 & -1.398400 & 0.777999 \\ 5 & 1 & 0 & -2.151645 & 0.038955 & 0.164281 \\ 6 & 6 & 0 & 1.295910 & -0.622131 & -0.002595 \\ 7 & 1 & 0 & 1.417337 & -1.131244 & 0.964064\end{array}$




$\begin{array}{rrrrrr}8 & 1 & 0 & 1.272729 & -1.400522 & -0.775993 \\ 9 & 1 & 0 & 2.151389 & 0.038433 & -0.166539 \\ 10 & 8 & 0 & 0.000119 & 1.426150 & 0.000022\end{array}$

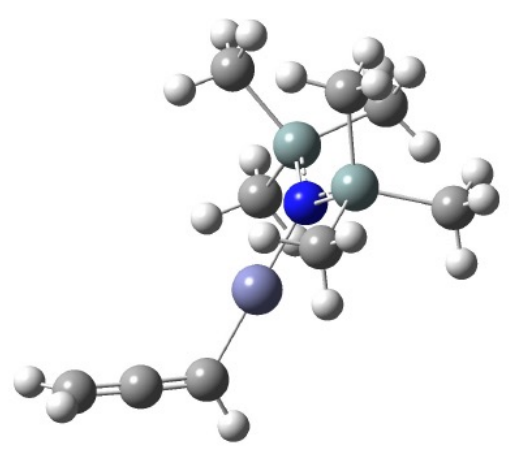

$\operatorname{AllZn}\left(A^{*}\right)$

Standard orientation:

$\begin{array}{lccccc}\text { Center } & \text { Atomic } & \text { Atomic } & \text { Coordinates (Angstroms) } & \\ \text { Number } & \text { Number } & \text { Type } & X & \text { Y } & \text { Z }\end{array}$

\begin{tabular}{|c|c|c|c|c|c|}
\hline & & & & & \\
\hline 1 & 6 & 0 & 0.958795 & -1.659295 & 0.788181 \\
\hline 2 & 7 & 0 & 1.225808 & -0.345031 & 0.663091 \\
\hline 3 & 6 & 0 & 2.592152 & -0.069438 & 1.203999 \\
\hline 4 & 6 & 0 & 2.997072 & -1.406332 & 1.916414 \\
\hline 5 & 8 & 0 & 1.959781 & -2.372268 & 1.453108 \\
\hline 6 & 6 & 0 & -0.161783 & -2.383518 & 0.350279 \\
\hline 7 & 6 & 0 & -1.232932 & -1.791603 & -0.334033 \\
\hline 8 & 8 & 0 & -2.293697 & -2.624773 & -0.703614 \\
\hline 9 & 6 & 0 & -3.265165 & -1.825676 & -1.501823 \\
\hline 10 & 6 & 0 & -2.758698 & -0.353197 & -1.318519 \\
\hline 11 & 7 & 0 & -1.406979 & -0.504987 & -0.701301 \\
\hline 12 & 30 & 0 & -0.045901 & 0.905580 & -0.259307 \\
\hline 13 & 6 & 0 & -4.681873 & -1.924289 & -0.893776 \\
\hline 14 & 6 & 0 & -4.864989 & -0.609589 & -0.149930 \\
\hline 15 & 6 & 0 & -3.803336 & 0.281798 & -0.406025 \\
\hline 16 & 6 & 0 & 3.666148 & 0.115076 & 0.136505 \\
\hline 17 & 6 & 0 & 4.656051 & -0.883899 & 0.231578 \\
\hline 18 & 6 & 0 & 4.393166 & -1.820780 & 1.401248 \\
\hline 19 & 6 & 0 & -5.925165 & -0.227497 & 0.688343 \\
\hline 20 & 6 & 0 & -5.915273 & 1.059977 & 1.262444 \\
\hline & & & $\mathrm{S} 22$ & & \\
\hline
\end{tabular}




$\begin{array}{rrrrrr}21 & 6 & 0 & -4.854900 & 1.952995 & 0.998658 \\ 22 & 6 & 0 & -3.790234 & 1.566570 & 0.160250 \\ 23 & 6 & 0 & 3.738173 & 1.108560 & -0.853289 \\ 24 & 6 & 0 & 4.814047 & 1.085460 & -1.763249 \\ 25 & 6 & 0 & 5.801190 & 0.080556 & -1.676963 \\ 26 & 6 & 0 & 5.727199 & -0.909819 & -0.676488 \\ 27 & 6 & 0 & 0.043285 & 2.898799 & -0.570458 \\ 28 & 6 & 0 & 0.822601 & 3.650959 & 0.186611 \\ 29 & 6 & 0 & 1.627846 & 4.353094 & 0.983840 \\ 30 & 1 & 0 & 2.545643 & 0.784837 & 1.888245 \\ 31 & 1 & 0 & 2.921630 & -1.375584 & 3.006653 \\ 32 & 1 & 0 & -0.200963 & -3.444456 & 0.551131 \\ 33 & 1 & 0 & -3.200862 & -2.198381 & -2.527720 \\ 34 & 1 & 0 & -2.667132 & 0.183536 & -2.270053 \\ 35 & 1 & 0 & -5.433125 & -2.041460 & -1.686929 \\ 36 & 1 & 0 & -4.754365 & -2.802398 & -0.241645 \\ 37 & 1 & 0 & 5.147125 & -1.693161 & 2.190216 \\ 38 & 1 & 0 & 4.393728 & -2.879178 & 1.115787 \\ 39 & 1 & 0 & -6.744288 & -0.912681 & 0.895999 \\ 40 & 1 & 0 & -6.729543 & 1.368383 & 1.914187 \\ 41 & 1 & 0 & -4.858402 & 2.943679 & 1.446705 \\ 42 & 1 & 0 & -2.972259 & 2.257243 & -0.034171 \\ 43 & 1 & 0 & 2.978213 & 1.883749 & -0.922088 \\ 44 & 1 & 0 & 4.884402 & 1.846680 & -2.536630 \\ 45 & 1 & 0 & 6.624994 & 0.072113 & -2.387149 \\ 46 & 1 & 0 & 6.491509 & -1.681536 & -0.613338 \\ 47 & 1 & 0 & -0.543996 & 3.387999 & -1.356146 \\ 48 & 1 & 0 & 2.664892 & 4.547845 & 0.715471 \\ 49 & 1 & 0 & 1.269075 & 4.778339 & 1.919423 \\ -----------------------------\end{array}$

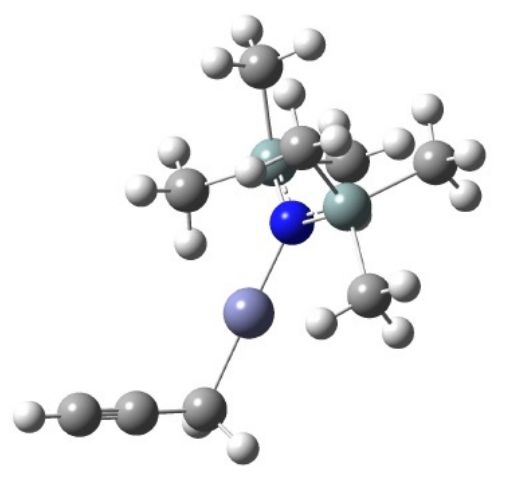

Pro-Zn(A) 
Standard orientation:

$\begin{array}{lrrrcr}\text { Center } & \text { Atomic } & \text { Atomic } & \text { Coordinates (Angstroms) } & \\ \text { Number } & \text { Number } & \text { Type } & \text { X } & \text { Y } & \text { Z }\end{array}$

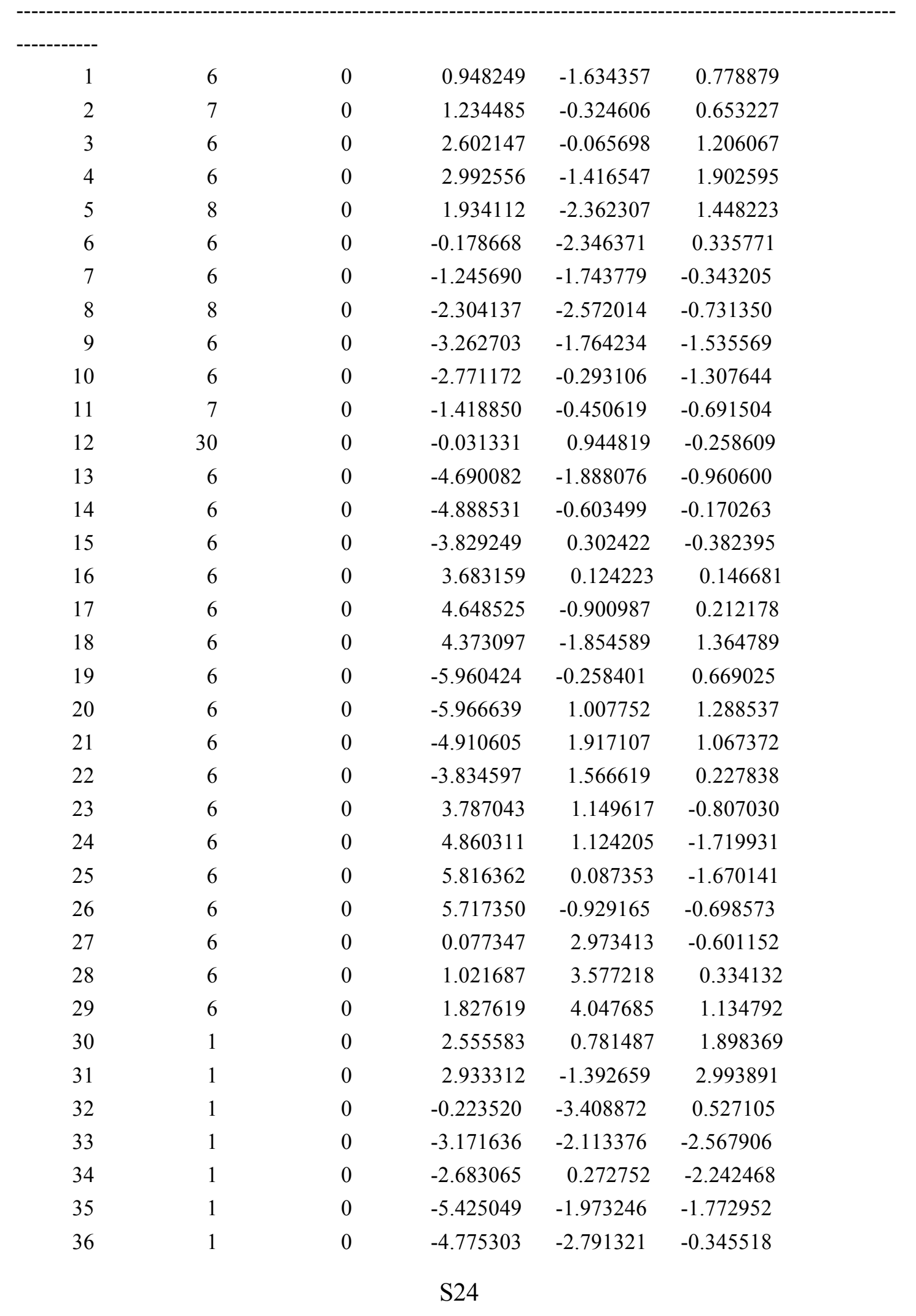




$\begin{array}{lllrrc}37 & 1 & 0 & 5.138625 & -1.760793 & 2.147276 \\ 38 & 1 & 0 & 4.343981 & -2.907126 & 1.059768 \\ 39 & 1 & 0 & -6.776832 & -0.956101 & 0.842915 \\ 40 & 1 & 0 & -6.790067 & 1.287126 & 1.941778 \\ 41 & 1 & 0 & -4.926871 & 2.891866 & 1.548818 \\ 42 & 1 & 0 & -3.022083 & 2.270866 & 0.062935 \\ 43 & 1 & 0 & 3.065821 & 1.962681 & -0.830418 \\ 44 & 1 & 0 & 4.956129 & 1.911910 & -2.463511 \\ 45 & 1 & 0 & 6.638656 & 0.077672 & -2.382093 \\ 46 & 1 & 0 & 6.462352 & -1.720999 & -0.657433 \\ 47 & 1 & 0 & 0.391153 & 3.149583 & -1.640273 \\ 48 & 1 & 0 & -0.922480 & 3.413354 & -0.477035 \\ 49 & 1 & 0 & 2.519431 & 4.488956 & 1.815070\end{array}$

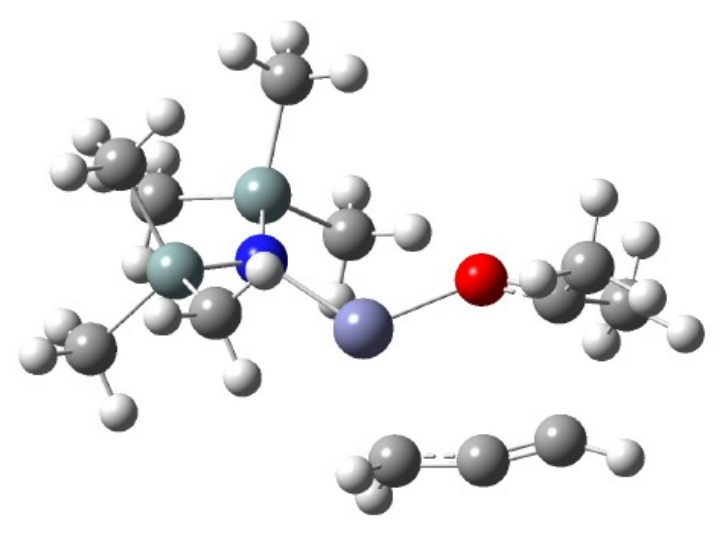

TS1

Standard orientation:

\begin{tabular}{lcccc} 
Center & Atomic & Atomic & \multicolumn{2}{c}{ Coordinates (Angstroms) } \\
Number & Number & Type & $X$ & $Y$ \\
- & & & &
\end{tabular}

$\begin{array}{rrrrrr}1 & 6 & 0 & 3.120803 & -1.579227 & -1.130039 \\ 2 & 7 & 0 & -1.255651 & 0.021127 & -0.038773 \\ 3 & 14 & 0 & -1.741973 & 1.708989 & -0.232262 \\ 4 & 14 & 0 & -2.335139 & -1.311536 & 0.377922 \\ 5 & 6 & 0 & -3.620967 & -0.817244 & 1.697064 \\ 6 & 1 & 0 & -4.294611 & -0.026267 & 1.341143 \\ 7 & 1 & 0 & -4.241876 & -1.682651 & 1.969964 \\ 8 & 1 & 0 & -3.126351 & -0.454611 & 2.608215 \\ 9 & 6 & 0 & -1.321283 & -2.761278 & 1.091383\end{array}$




$\begin{array}{lrrrrr}10 & 1 & 0 & -0.617653 & -3.180784 & 0.357735 \\ 11 & 1 & 0 & -0.749945 & -2.449705 & 1.977407 \\ 12 & 1 & 0 & -1.990395 & -3.577232 & 1.399218 \\ 13 & 6 & 0 & -3.281057 & -1.977243 & -1.139666 \\ 14 & 1 & 0 & -2.580146 & -2.317900 & -1.914994 \\ 15 & 1 & 0 & -3.925830 & -2.826309 & -0.869741 \\ 16 & 1 & 0 & -3.914830 & -1.197723 & -1.583084 \\ 17 & 6 & 0 & -0.427779 & 2.627587 & -1.260288 \\ 18 & 1 & 0 & -0.334076 & 2.194169 & -2.266693 \\ 19 & 1 & 0 & -0.707187 & 3.683831 & -1.381119 \\ 20 & 1 & 0 & 0.559919 & 2.600535 & -0.779114 \\ 21 & 6 & 0 & -3.405218 & 1.885058 & -1.150747 \\ 22 & 1 & 0 & -4.235745 & 1.425910 & -0.598006 \\ 23 & 1 & 0 & -3.650679 & 2.947293 & -1.294445 \\ 24 & 1 & 0 & -3.356984 & 1.413213 & -2.141528 \\ 25 & 6 & 0 & -1.897782 & 2.611942 & 1.439933 \\ 26 & 1 & 0 & -2.669350 & 2.150724 & 2.070489 \\ 27 & 1 & 0 & -0.946492 & 2.569521 & 1.988387 \\ 28 & 1 & 0 & -2.162613 & 3.669986 & 1.298728 \\ 29 & 30 & 0 & 0.617780 & -0.393739 & -0.349125 \\ 30 & 8 & 0 & 2.114578 & 0.677447 & 0.443629 \\ 31 & 6 & 0 & 3.847207 & 0.001927 & 1.993173 \\ 32 & 1 & 0 & 3.268436 & -0.895975 & 2.228228 \\ 33 & 1 & 0 & 4.913019 & -0.241063 & 1.990795 \\ 34 & 1 & 0 & 3.664136 & 0.743153 & 2.787462 \\ 35 & 6 & 0 & 4.277920 & 1.662029 & 0.020700 \\ 36 & 1 & 0 & 5.337736 & 1.397550 & 0.067863 \\ 37 & 1 & 0 & 3.984415 & 1.823110 & -1.020129 \\ 38 & 1 & 0 & 4.135159 & 2.608316 & 0.566268 \\ 39 & 6 & 0 & 1.786818 & -1.827243 & -1.500913 \\ 40 & 1 & 0 & 1.536956 & -1.597058 & -2.541784 \\ 41 & 1 & 0 & 1.381459 & -2.792392 & -1.179381 \\ 42 & 6 & 0 & 4.179249 & -1.126006 & -0.630244 \\ 43 & 1 & 0 & 5.247281 & -1.149636 & -0.528698 \\ 44 & 6 & 0 & 3.391316 & 0.606759 & 0.670262\end{array}$




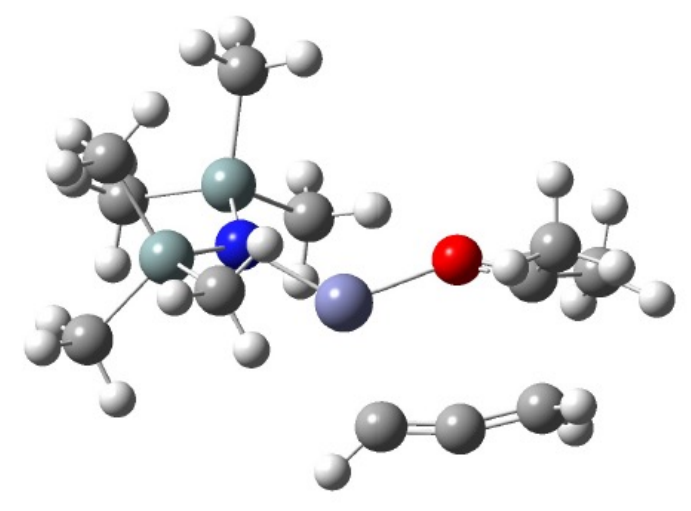

TS1*

Standard orientation:

\begin{tabular}{lccccc} 
Center & Atomic & Atomic & \multicolumn{2}{c}{ Coordinates (Angstroms) } \\
Number & Number & Type & X & $Y$ & $Z$ \\
- & & & &
\end{tabular}

$\begin{array}{rrrrrr}1 & 6 & 0 & 3.015461 & -1.503802 & -1.237478 \\ 2 & 7 & 0 & -1.276007 & 0.021191 & -0.041244 \\ 3 & 14 & 0 & -1.818611 & 1.695654 & -0.201197 \\ 4 & 14 & 0 & -2.305845 & -1.361444 & 0.337351 \\ 5 & 6 & 0 & -3.645511 & -0.941168 & 1.627711 \\ 6 & 1 & 0 & -4.337129 & -0.167317 & 1.268393 \\ 7 & 1 & 0 & -4.242059 & -1.834672 & 1.861807 \\ 8 & 1 & 0 & -3.192647 & -0.582291 & 2.561659 \\ 9 & 6 & 0 & -1.235748 & -2.764125 & 1.058653 \\ 10 & 1 & 0 & -0.474741 & -3.115809 & 0.346540 \\ 11 & 1 & 0 & -0.722735 & -2.438954 & 1.974993 \\ 12 & 1 & 0 & -1.861294 & -3.629850 & 1.318555 \\ 13 & 6 & 0 & -3.188258 & -2.051913 & -1.207346 \\ 14 & 1 & 0 & -2.458631 & -2.362352 & -1.968662 \\ 15 & 1 & 0 & -3.807549 & -2.925421 & -0.956336 \\ 16 & 1 & 0 & -3.840104 & -1.293317 & -1.661053 \\ 17 & 6 & 0 & -0.482917 & 2.692241 & -1.121455 \\ 18 & 1 & 0 & -0.339733 & 2.316630 & -2.145176 \\ 19 & 1 & 0 & -0.777274 & 3.748649 & -1.196420 \\ 20 & 1 & 0 & 0.485765 & 2.654921 & -0.603501 \\ 21 & 6 & 0 & -3.438803 & 1.834467 & -1.198718 \\ 22 & 1 & 0 & -4.274455 & 1.324413 & -0.700655 \\ 23 & 1 & 0 & -3.723094 & 2.889079 & -1.325734 \\ 24 & 1 & 0 & -3.321217 & 1.393295 & -2.197829 \\ 25 & 6 & 0 & -2.100038 & 2.532922 & 1.488985\end{array}$




$\begin{array}{rrrrrr}26 & 1 & 0 & -2.876364 & 2.014416 & 2.066850 \\ 27 & 1 & 0 & -1.175269 & 2.522300 & 2.082421 \\ 28 & 1 & 0 & -2.412269 & 3.580180 & 1.364925 \\ 29 & 30 & 0 & 0.612507 & -0.310613 & -0.328395 \\ 30 & 8 & 0 & 2.146485 & 0.736204 & 0.362362 \\ 31 & 6 & 0 & 3.724318 & -0.238626 & 1.917912 \\ 32 & 1 & 0 & 3.155170 & -1.173245 & 1.967261 \\ 33 & 1 & 0 & 4.790570 & -0.456603 & 2.030344 \\ 34 & 1 & 0 & 3.408140 & 0.392692 & 2.761612 \\ 35 & 6 & 0 & 4.376811 & 1.647868 & 0.244306 \\ 36 & 1 & 0 & 5.424558 & 1.335362 & 0.286110 \\ 37 & 1 & 0 & 4.144581 & 2.031025 & -0.753860 \\ 38 & 1 & 0 & 4.232652 & 2.468426 & 0.963764 \\ 39 & 6 & 0 & 3.420172 & 0.516580 & 0.620950 \\ 40 & 6 & 0 & 4.195148 & -0.960820 & -0.776787 \\ 41 & 1 & 0 & 4.803240 & -1.543020 & -0.086413 \\ 42 & 1 & 0 & 4.752971 & -0.318464 & -1.457209 \\ 43 & 6 & 0 & 1.779800 & -1.731352 & -1.475376 \\ 44 & 1 & 0 & 1.243648 & -2.422236 & -2.115007\end{array}$

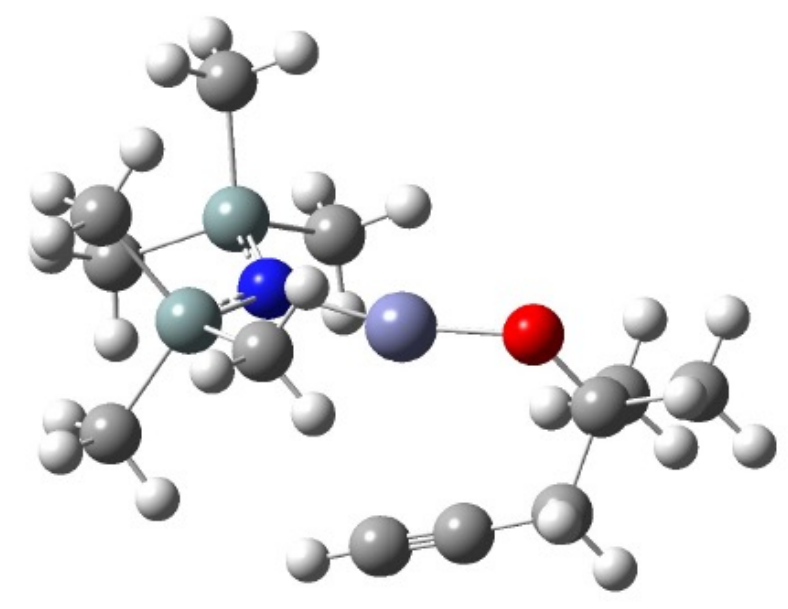

B*

Standard orientation:

\begin{tabular}{lcccc} 
Center & Atomic & Atomic & \multicolumn{2}{c}{ Coordinates (Angstroms) } \\
Number & Number & Type & $X$ & $Y$ \\
-
\end{tabular}




7
14
14
6
1
1
1
6
1
1
1
6
1
1
1
6
1
1
1
6
1
1
1
6
6
1
1
1
1
6
6
1
1
1
1
1
1
1
1
1
1
1
1
1
1
1
1
1

1.250346

0.034122

$-0.131705$

2.032433

1.605750

0.110212

2.089415

$-1.524742$

$-0.211454$

3.740196

$-1.399786$

$-1.153428$

4.441908

$-0.703625$

$-0.675299$

4.227635

$-2.384492$

$-1.192183$

3.578807

$-1.062126$

$-2.185751$

1.006596

$-2.799672$

$-1.123944$

0.060788

$-3.001454$

$-0.600303$

0.769644

$-2.467081$

$-2.144240$

1.539701

$-3.757543$

$-1.202963$

2.456503

$-2.216941$

1.526492

1.526040

$-2.370993$

2.090407

2.976474

$-3.183842$

1.464902

3.089795

$-1.529247$

2.102442

0.702584

2.872664

0.611920

0.185351

2.570464

1.533784

1.161731

3.853299

0.799807

$-0.051982$

3.020290

$-0.175155$

3.341236

1.545702

1.492511

4.161418

0.856025

1.252555

3.781351

2.541074

1.647662

2.894634

1.221394

2.441986

2.869823

2.243934

$-1.477414$

3.674674

1.57211

$-1.802564$

2.142531

2.316164

$-2.298169$

3.304403

3.242076

$-1.322243$

$-0.647598$

0.047740

$-0.351925$

$-2.382459$

0.132687

$-1.022537$

$-4.756174$

$-0.117957$

$-1.364572$

$-4.566545$

$-1.07274$

$-1.869626$

$-5.749346$

$-0.154676$

$-0.895862$

$-4.756210$

0.673009

$-2.123242$

$-3.862095$

1.544879

0.333149

$-4.854557$

1.622556

0.797704

$-3.106526$

1.732232

1.107709

$-3.773507$

2.327098

$-0.430014$

$-3.647138$

0.162556

$-0.327764$

$-3.693045$

$-0.976523$

0.759963

$-3.702555$

$-1.945381$

0.242203

$-4.622753$

$-0.901356$

1.339862

$-1.550567$

$-0.913178$

2.400112

$-0.715860$

$-0.895630$

3.065419 


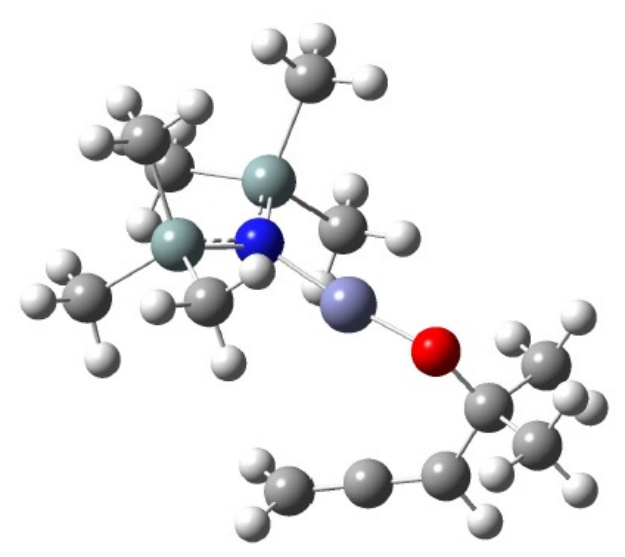

B

Standard orientation:

\begin{tabular}{|c|c|c|c|c|c|}
\hline \multirow{2}{*}{$\begin{array}{l}\text { Center } \\
\text { Number }\end{array}$} & \multirow{2}{*}{$\begin{array}{l}\text { Atomic } \\
\text { Number }\end{array}$} & \multirow{2}{*}{$\begin{array}{l}\text { Atomic } \\
\text { Type }\end{array}$} & \multicolumn{3}{|c|}{ Coordinates (Angstroms) } \\
\hline & & & $\mathrm{X}$ & $\mathrm{Y}$ & $\mathrm{Z}$ \\
\hline
\end{tabular}

$\begin{array}{rrrrrc}1 & 6 & 0 & -2.644620 & -0.616165 & 1.793406 \\ 2 & 7 & 0 & 1.271086 & 0.028998 & -0.078617 \\ 3 & 14 & 0 & 2.011652 & 1.626749 & 0.086585 \\ 4 & 14 & 0 & 2.118558 & -1.514314 & -0.234148 \\ 5 & 6 & 0 & 3.463741 & -1.460741 & -1.580527 \\ 6 & 1 & 0 & 4.251521 & -0.733982 & -1.339885 \\ 7 & 1 & 0 & 3.943016 & -2.443436 & -1.695471 \\ 8 & 1 & 0 & 3.030725 & -1.178948 & -2.549707 \\ 9 & 6 & 0 & 0.830664 & -2.829896 & -0.717181 \\ 10 & 1 & 0 & 0.000976 & -2.867326 & 0.004985 \\ 11 & 1 & 0 & 0.408760 & -2.639418 & -1.714301 \\ 12 & 1 & 0 & 1.282428 & -3.831190 & -0.738891 \\ 13 & 6 & 0 & 2.928161 & -2.071700 & 1.397931 \\ 14 & 1 & 0 & 2.180093 & -2.144655 & 2.199578 \\ 15 & 1 & 0 & 3.398708 & -3.058986 & 1.283452 \\ 16 & 1 & 0 & 3.702324 & -1.366208 & 1.725265 \\ 17 & 6 & 0 & 0.792430 & 2.760630 & 1.010265 \\ 18 & 1 & 0 & 0.593846 & 2.378854 & 2.021465 \\ 19 & 1 & 0 & 1.201888 & 3.775487 & 1.111129 \\ 20 & 1 & 0 & -0.170559 & 2.854031 & 0.485967 \\ 21 & 6 & 0 & 3.637096 & 1.572451 & 1.075854 \\ 22 & 1 & 0 & 4.398910 & 0.952262 & 0.584876 \\ & & & & & \\ 12 & & & & & \end{array}$




\begin{tabular}{rrrrrr}
23 & 1 & 0 & 4.051376 & 2.585990 & 1.173915 \\
24 & 1 & 0 & 3.475023 & 1.174642 & 2.086154 \\
25 & 6 & 0 & 2.382237 & 2.412036 & -1.609825 \\
26 & 1 & 0 & 3.085028 & 1.792832 & -2.183886 \\
27 & 1 & 0 & 1.463981 & 2.513186 & -2.205381 \\
28 & 1 & 0 & 2.824655 & 3.412662 & -1.499491 \\
29 & 30 & 0 & -0.634300 & -0.019749 & -0.218464 \\
30 & 8 & 0 & -2.340745 & 0.169469 & -0.930736 \\
31 & 6 & 0 & -4.510117 & -0.860844 & -1.244299 \\
32 & 1 & 0 & -4.069101 & -1.863433 & -1.195517 \\
33 & 1 & 0 & -5.549798 & -0.914903 & -0.893775 \\
34 & 1 & 0 & -4.507089 & -0.530911 & -2.289810 \\
35 & 6 & 0 & -4.282776 & 1.556644 & -0.476572 \\
36 & 1 & 0 & -5.314160 & 1.578683 & -0.099373 \\
37 & 1 & 0 & -3.679385 & 2.258380 & 0.112048 \\
38 & 1 & 0 & -4.282472 & 1.889051 & -1.521429 \\
39 & 6 & 0 & -3.670533 & 0.132356 & -0.399689 \\
40 & 6 & 0 & -1.521605 & -0.883823 & 2.454688 \\
41 & 1 & 0 & -1.009623 & -0.122772 & 3.042646 \\
42 & 1 & 0 & -1.112754 & -1.893029 & 2.499099 \\
43 & 6 & 0 & -3.714315 & -0.329372 & 1.076990 \\
44 & 1 & 0 & -4.706082 & -0.406683 & 1.526099 \\
\hline----------------------------------------------------------------------------------
\end{tabular}

\section{Tables of the Computed Energies}

\begin{tabular}{|c|c|c|c|c|}
\hline Structure & $\mathrm{E}_{0}$ & $\mathrm{H}_{298}$ & $\mathrm{G}_{298}$ & TCGFE \\
\hline acetone & -193.049363 & -193.043103 & -193.077348 & 0.056146 \\
\hline $\mathbf{A}$ & -483.453917 & -483.431187 & -483.504634 & 0.227356 \\
\hline $\mathbf{A}^{*}$ & -483.463005 & -483.440099 & -483.514868 & 0.225978 \\
\hline TS1 & -676.508673 & -676.480538 & -676.566115 & 0.306334 \\
\hline TS1* & -676.511016 & -676.483306 & -676.567365 & 0.308223 \\
\hline B & -676.538926 & -676.511319 & -676.595118 & 0.311555 \\
\hline B* & -676.534767 & -676.507087 & -676.590739 & 0.311792 \\
\hline
\end{tabular}

$\mathrm{E}_{0}$ : Sum of electronic and zero-point Energies

$\mathrm{H}_{298}$ : Sum of electronic and thermal Enthalpies

$\mathrm{G}_{298}$ : Sum of electronic and thermal Free Energies

TCGFE : Thermal Correction to Gibbs Free Energy 


\section{References}

1. Y. Cui; W. Li; T. Sato; Y. Yamashita; S. Kobayashi, Adv. Synth. Catal. 2013, 355, 1193.

2. W. Miao, L. W. Chung, Y-D. Wu and T. H. Chan, J. Am. Chem. Soc. 2004, 126, 13326.

3. D. R. Fandrick, J. Saha, K. R. Fandrick, S. Sanyal, J. Ogikubo, H. Lee, F. Roschangar, J. J. Song and C. H. Senanayake, Org. Lett., 2011, 13, 5616.

4. K. Tonogaki, K. Itami and J. Yoshida, J. Am. Chem. Soc., 2006, 128, 1464.

5. D. -Y. Lee and J. F. Hartwig, Org. Lett., 2005, 7, 1169.

6. S. Kobayashi, R. Matsubara, Y. Nakamura, H. Kitagawa and M. Sugiura, J. Am. Chem. Soc., 2003, 125, 2507.

7. H. F. Bettinger, M. Filthaus, H. Bornemann and I. M. Oppel, Angew. Chem., Int. Ed., 2008, 47, 4744.

8. J. S. Schneekloth, M. Pucheault and C. M. Crews, Eur. J. Org. Chem., 2007, 40.

9. H. Nakamura, T. Kamakura, M. Ishikura and J. -F. Biellmann, J. Am. Chem. Soc., 2004, 126, 5958.

10. S. Shi, L. Xu, K. Oisaki, M. Kanai, M. Shibasaki, J. Am. Chem. Soc. 2010, 132, 6638.

11. M. Iyoda, Y. Kanao, M. Nishizaki and M. Oda, Bull. Chem. Soc. Jpn., 1989, 12, 3380 .

12. E. Yoneda, S-W. Zhang, D-Y. Zhou, K. Onitsuka and S. Takahashi, J. Org. Chem. 2003, 68, 8571 .

13. H. Tanaka, T. Hamatani, S. Yamashita and S. Torii, Chem. Lett. 1986, 9, 1461.

14. C. M. Yu, C. Kim and J. H. Kweon, Chem. Comm. 2004, 2494.

15. X. Ma, J.-X. Wang, S. Li, K.-H. Wang, D. Huang, Tetrahedron, 2009, 65, 8683.

16. D. S. Barnett, S. E. Schaus, Org. Lett., 2011, 13, 4020.

17. K. C. Harper, M. S. Sigman, Science 2011, 333, 1875.

18. T. Jumreang, B. D. Gregory, J. Am. Chem. Soc. 2008, 130, 5050.

19. E. Hernandez, C. H. Burgos, E. Alicea, J. A. Soderquist, Org. Lett., 2006, 8, 4089.

20. M. L. Maddess, M. Lautens, Org. Lett., 2005, 7, 3557.

21. J. A. Cabezas, L. X. Alvarez, Tetrahedron Lett, 1998, 39, 3935.

22. L. R. Reddy, Org. Lett, 2012, 14, 1142.

23. Gaussian 09, Revision B.01, Frisch, M. J.; Trucks, G. W.; Schlegel, H. B.;

Scuseria, G. E.; Robb, M. A.; Cheeseman, J. R.; Scalmani, G.; Barone, V.;

Mennucci, B.; Petersson, G. A.; Nakatsuji, H.; Caricato, M.; Li, X.; Hratchian, H. P.; Izmaylov, A. F.; Bloino, J.; Zheng, G.; Sonnenberg, J. L.; Hada, M.; Ehara, M.; Toyota, K.; Fukuda, R.; Hasegawa, J.; Ishida, M.; Nakajima, T.; Honda, Y.; Kitao, O.; Nakai, H.; Vreven, T.; Montgomery, J. A., Jr.; Peralta, J. E.; Ogliaro, F.; Bearpark, M.; Heyd, J. J.; Brothers, E.; Kudin, K. N.; Staroverov, V. N.; Kobayashi, R.; Normand, J.; Raghavachari, K.; Rendell, A.; Burant, J. C.; Iyengar, S. S.; Tomasi, J.; Cossi, M.; Rega, N.; Millam, N. J.; Klene, M.; Knox, J. E.; Cross, J. B.; Bakken, V.; Adamo, C.; Jaramillo, J.; Gomperts, R.; Stratmann, R. E.; Yazyev, O.; Austin, A. J.; Cammi, R.; Pomelli, C.; Ochterski, J. W.; Martin, R. L.; Morokuma, K.; Zakrzewski, V. G.; Voth, G. A.; Salvador, P.; Dannenberg, J. J.; Dapprich, S.; Daniels, A. D.; Farkas, Ö.; Foresman, J. B.; Ortiz, J. V.; Cioslowski, J.; Fox, D. J. Gaussian, Inc., Wallingford CT, 2009. 
VIII. Spectra
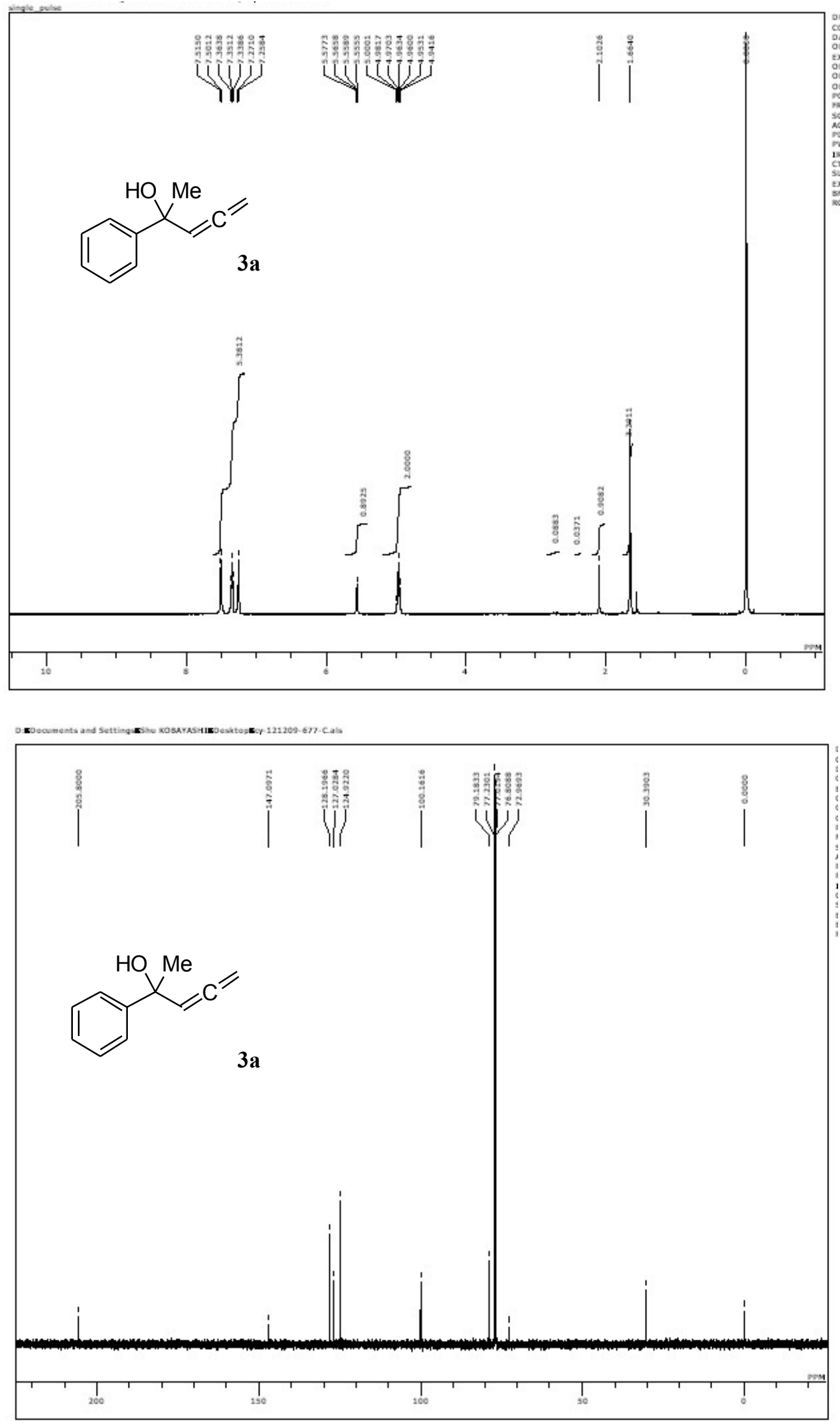

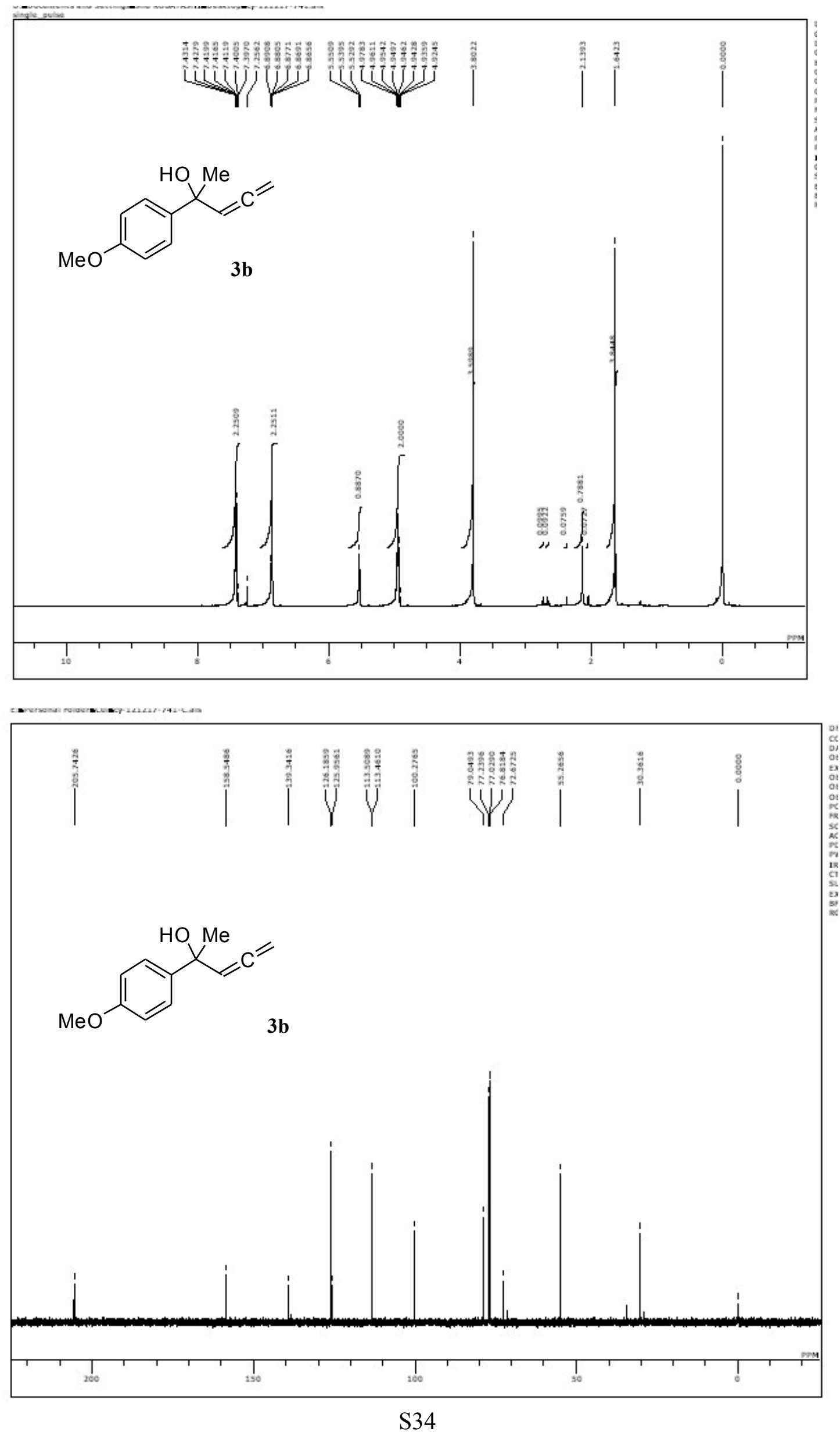

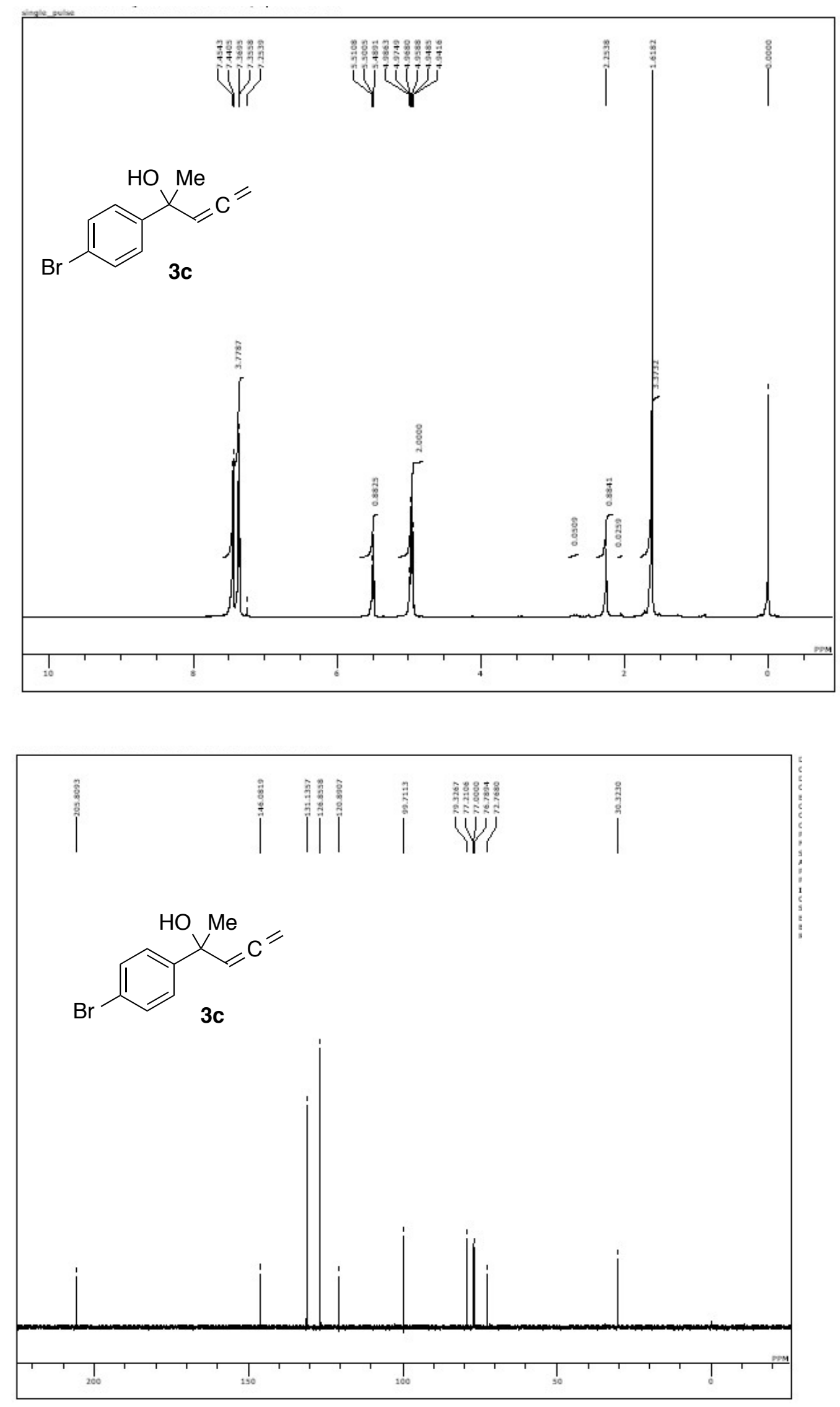

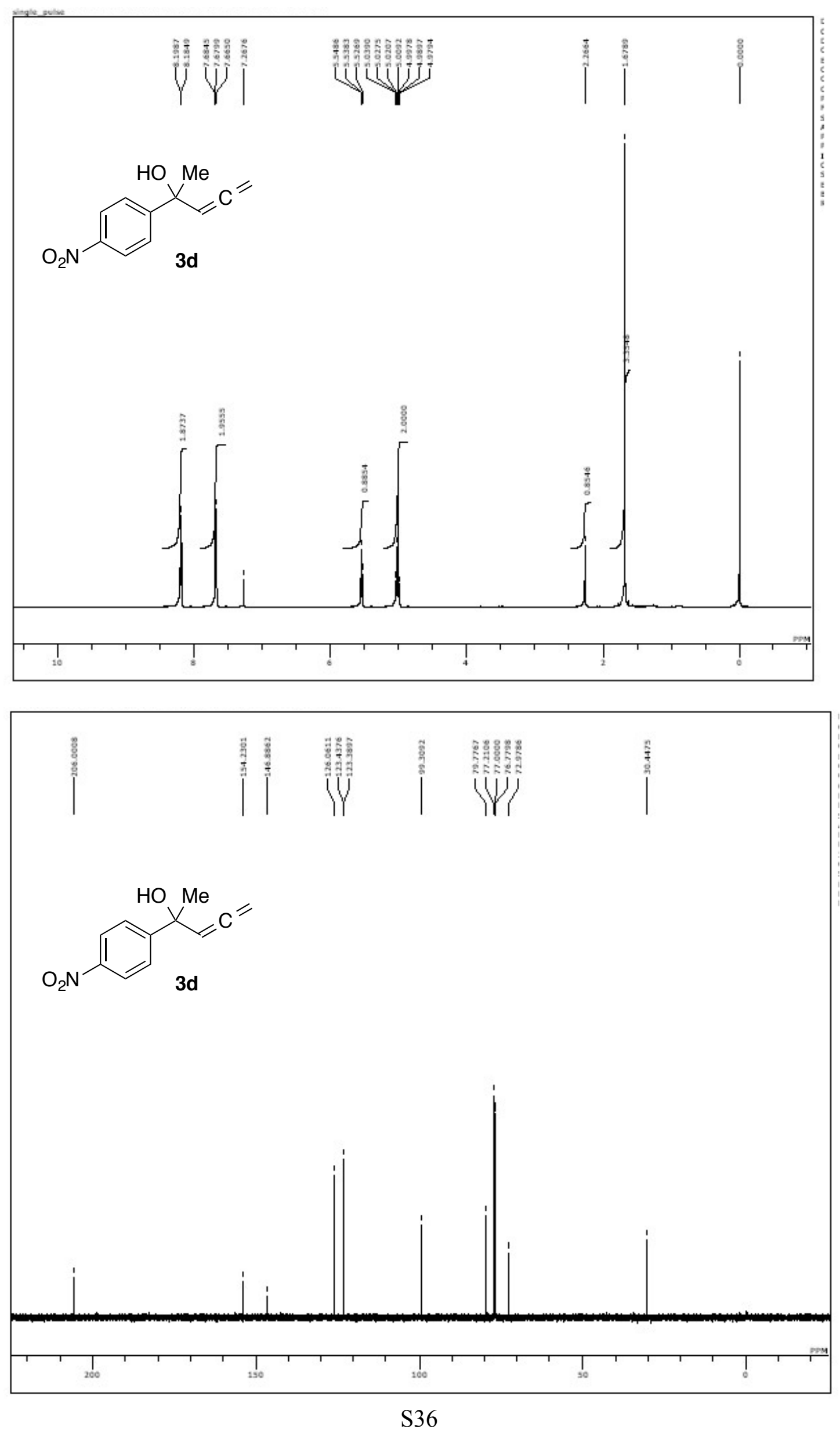

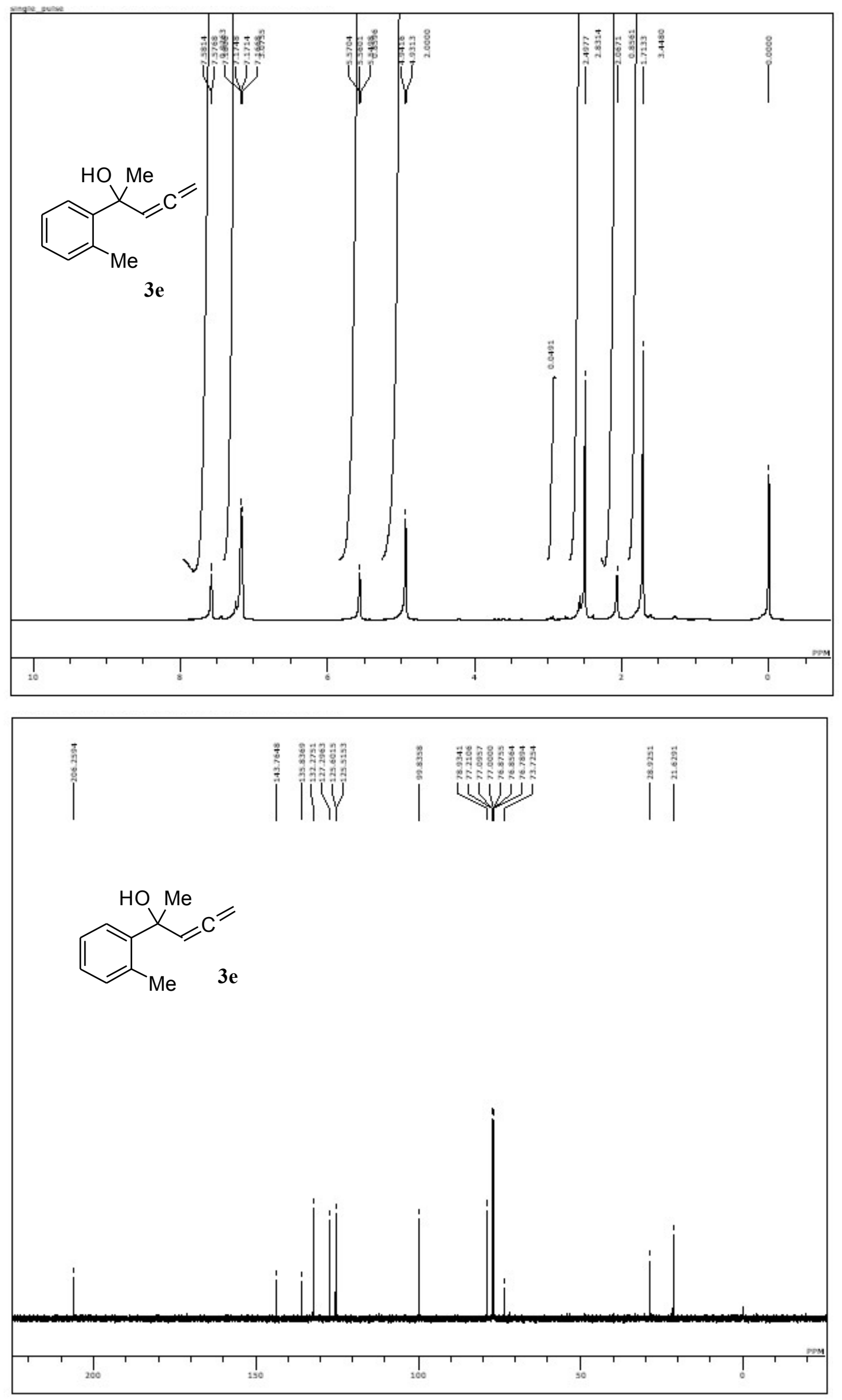


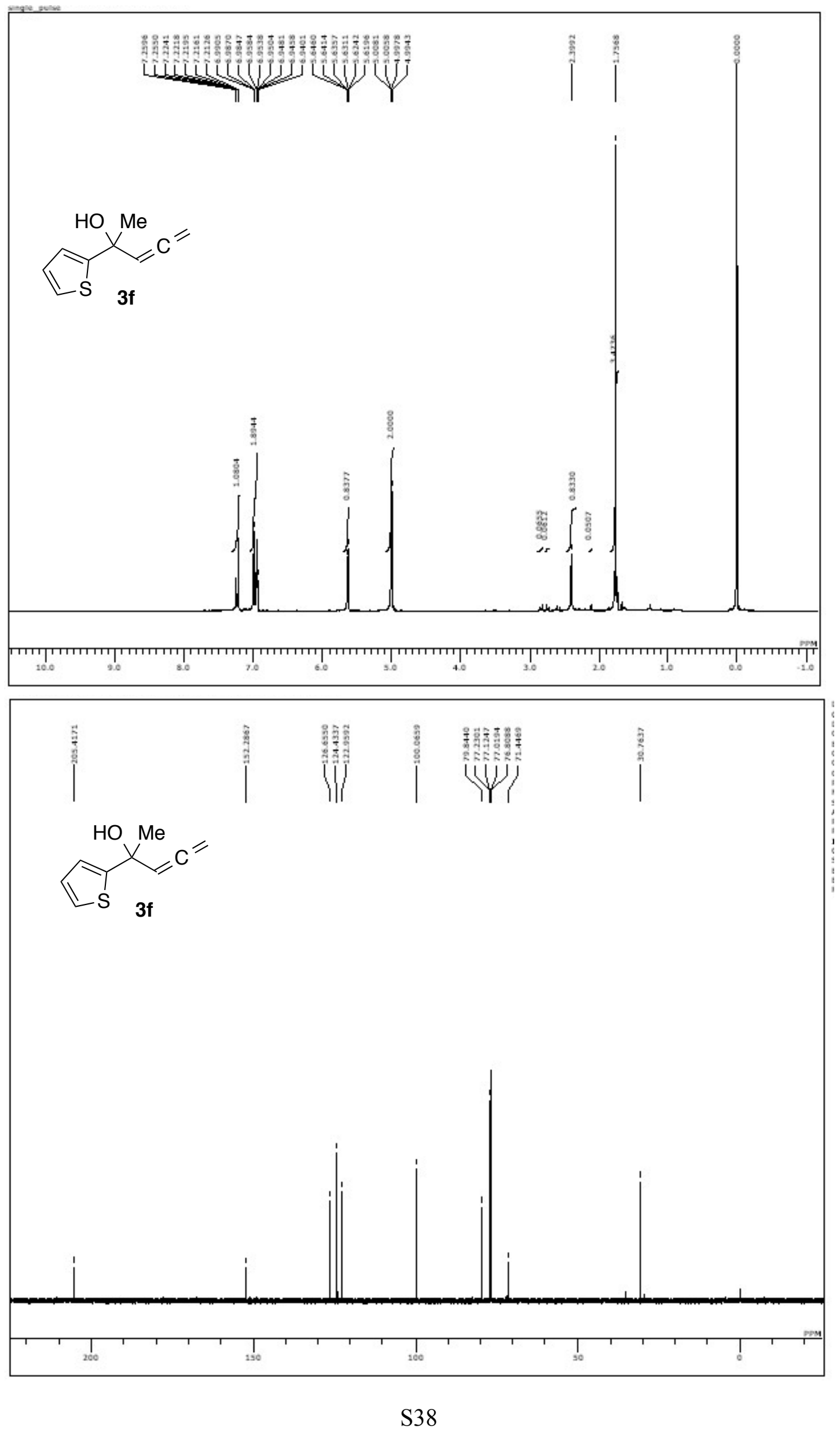



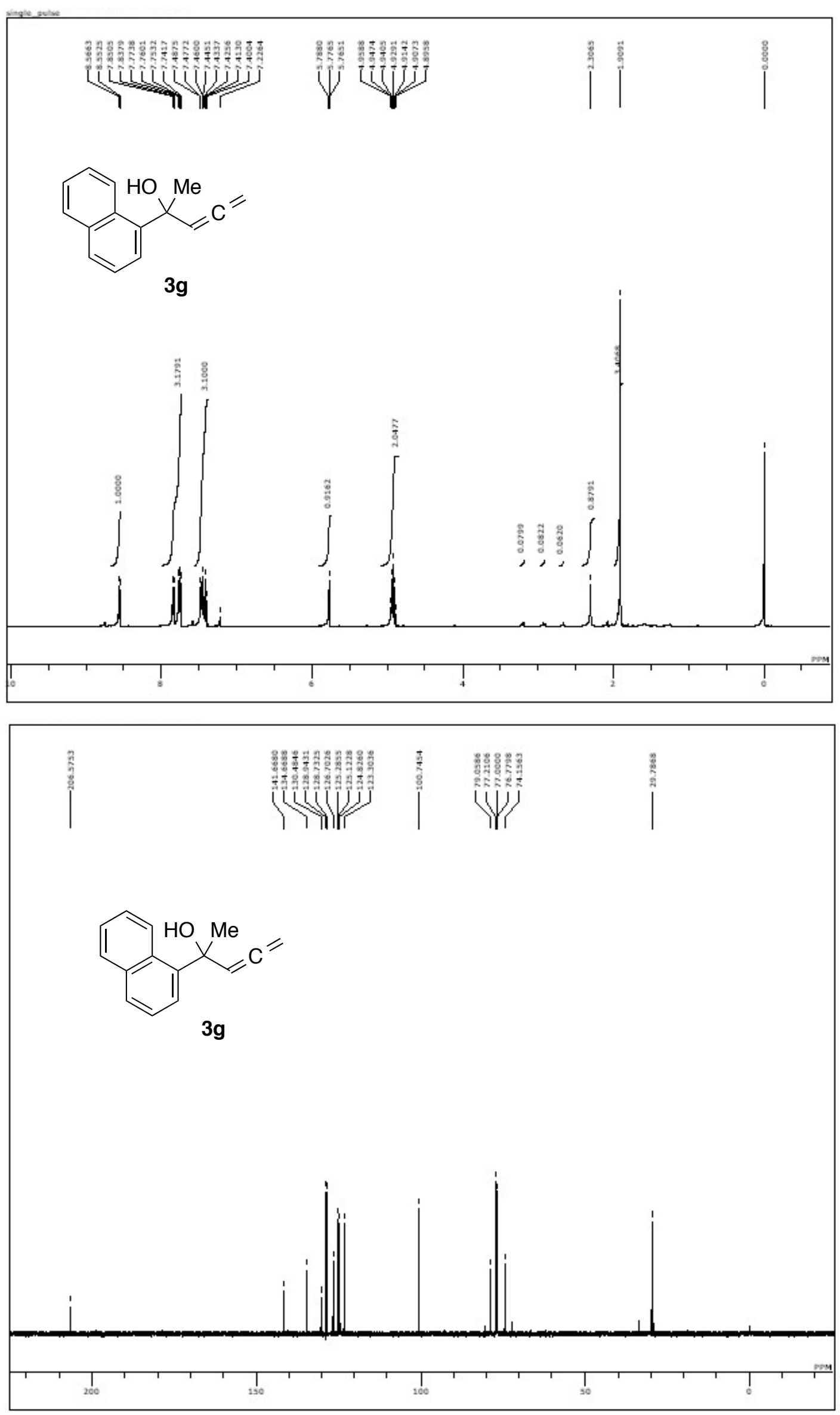

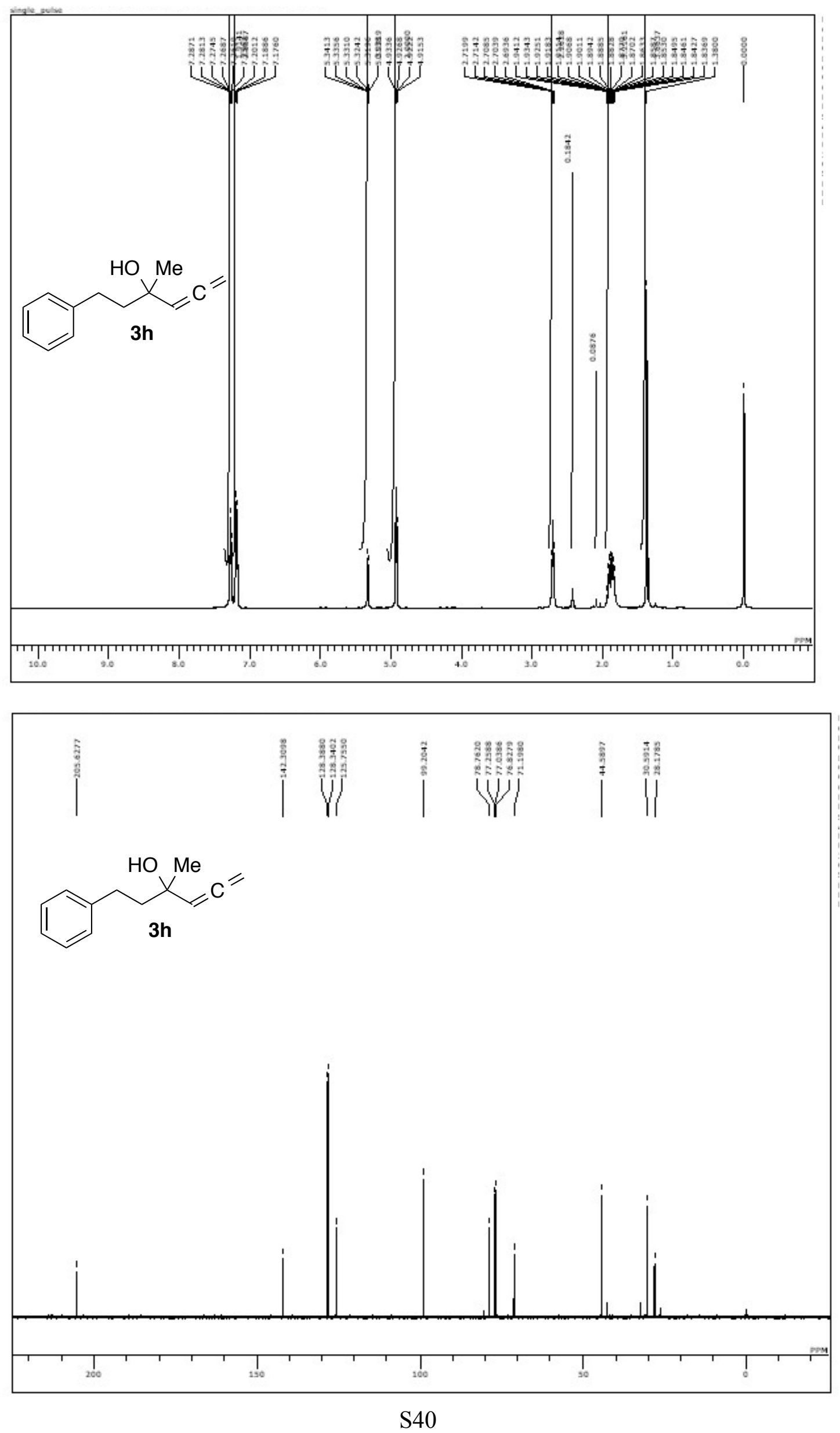


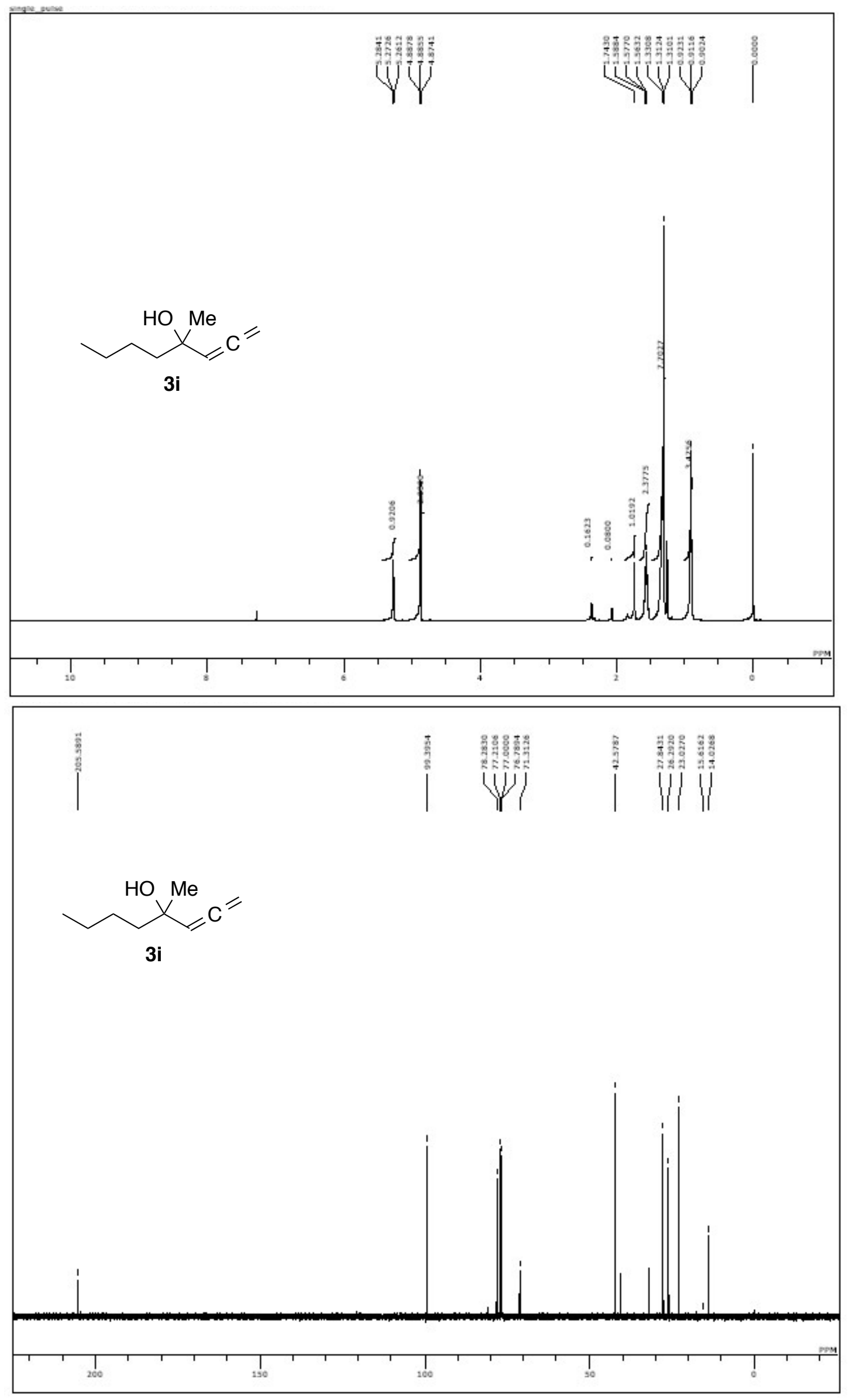



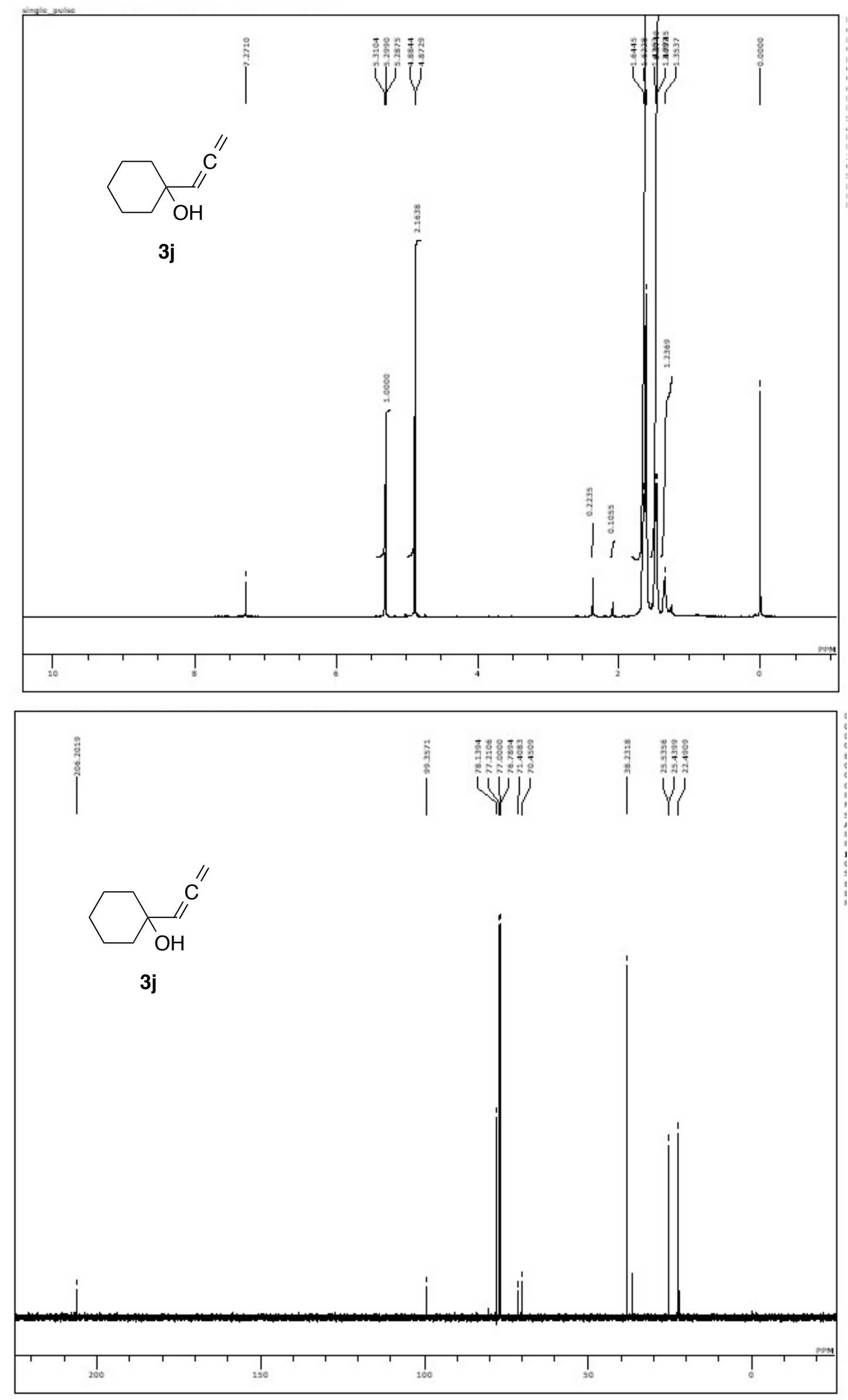
<smiles>CCC(O)(CC)c1ccccc1</smiles><smiles>C1CCCC1</smiles>

$3 k$

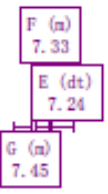

\begin{tabular}{|l|l|}
\hline A ( $)$ \\
5.57 \\
\hline 5
\end{tabular}
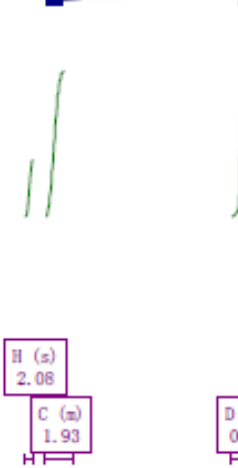

\begin{aligned} $\mathrm{C}(\mathrm{a}) \\ 1.93 \\$\hline\end{aligned}
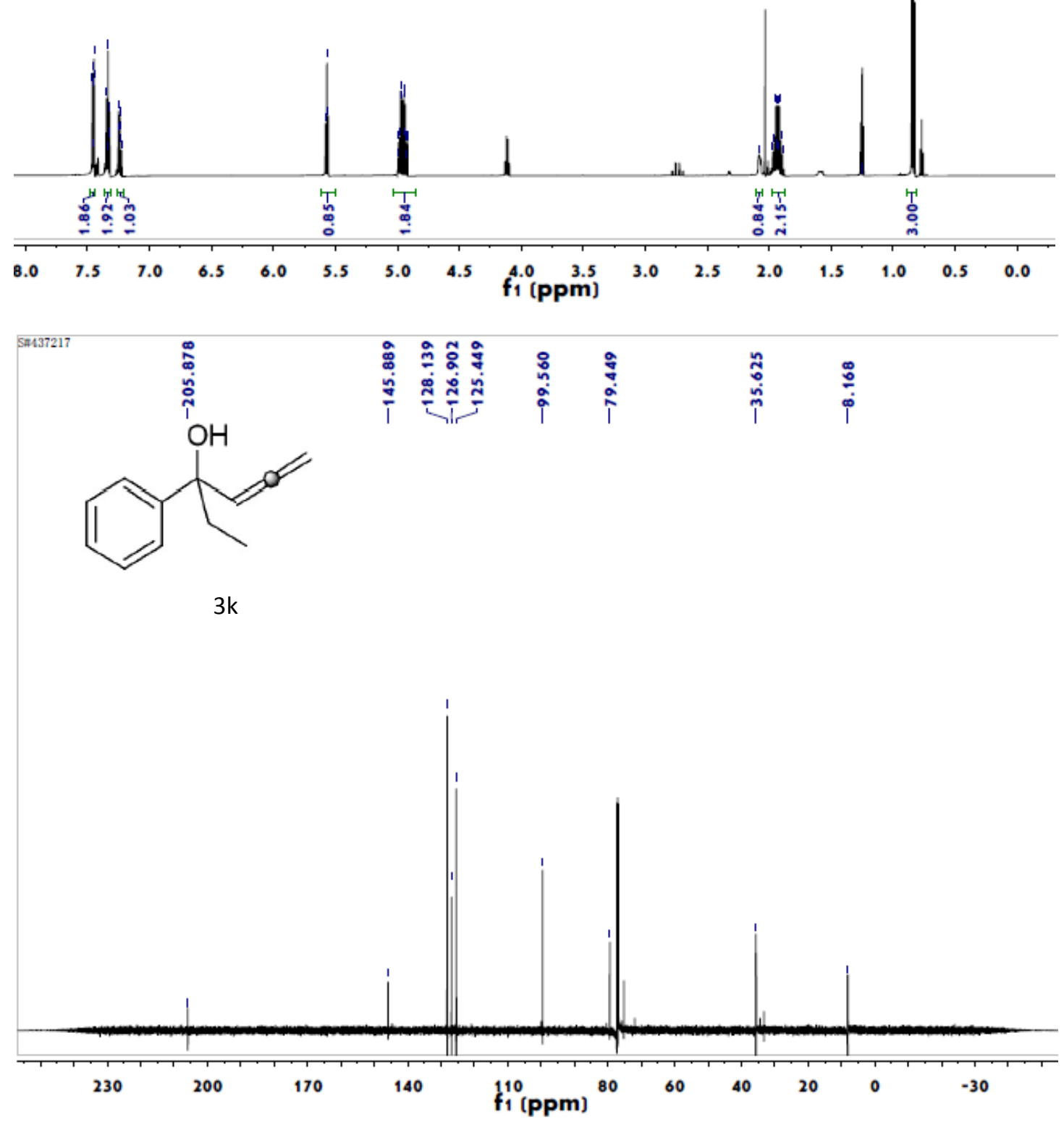


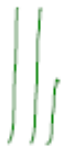<smiles>C=CC(O)(CC(C)C)c1ccccc1</smiles>

31

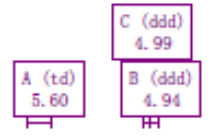
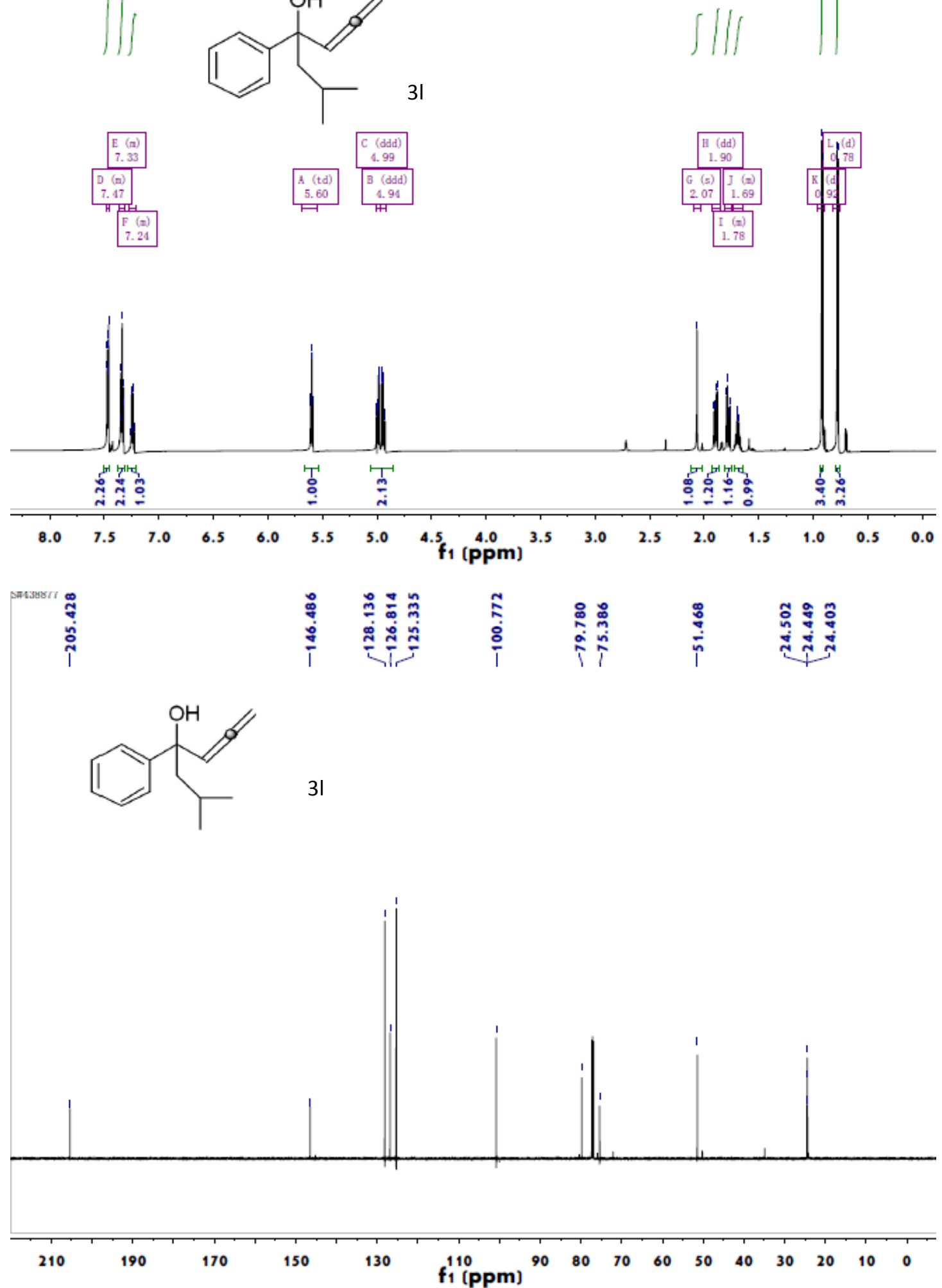

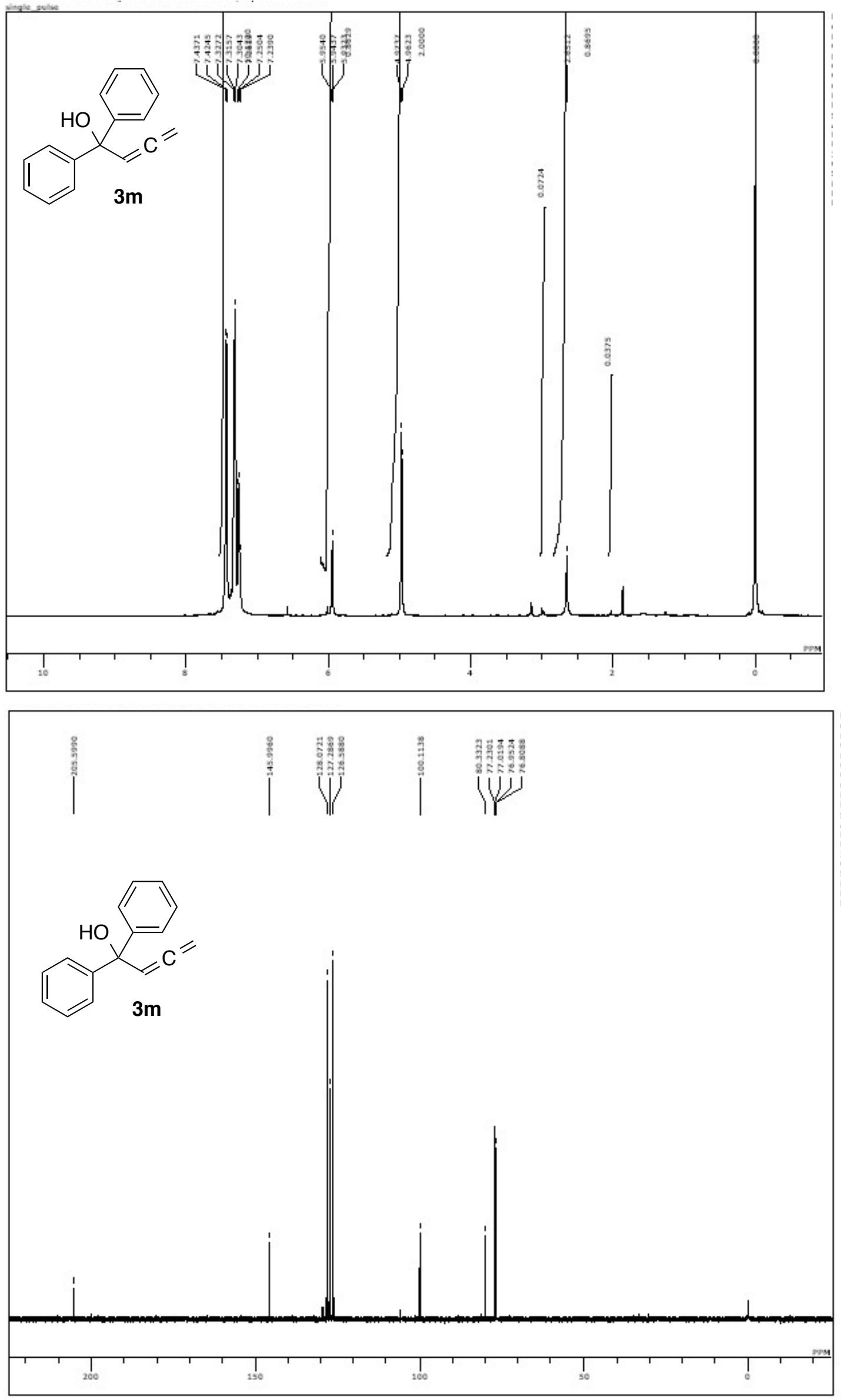

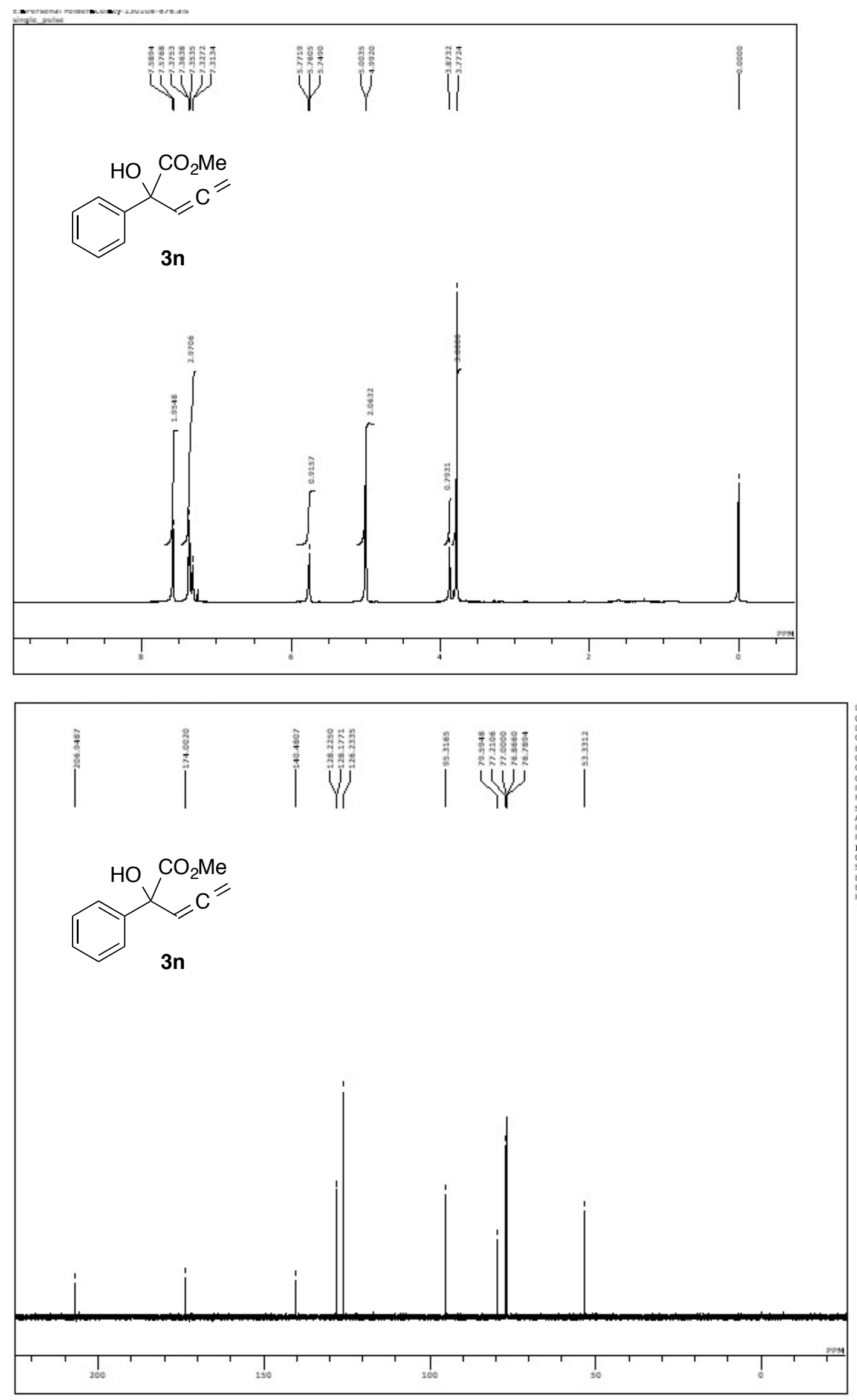

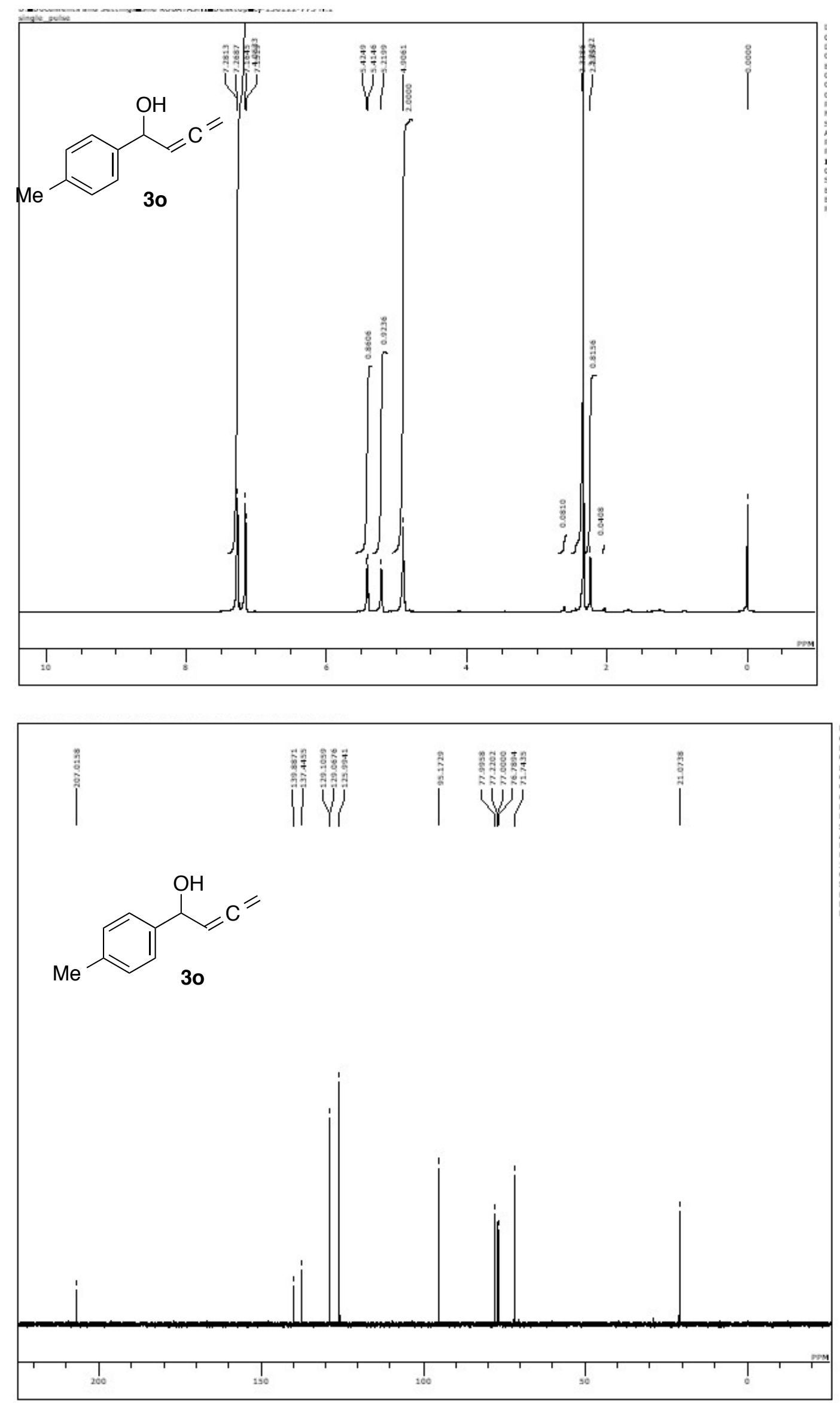

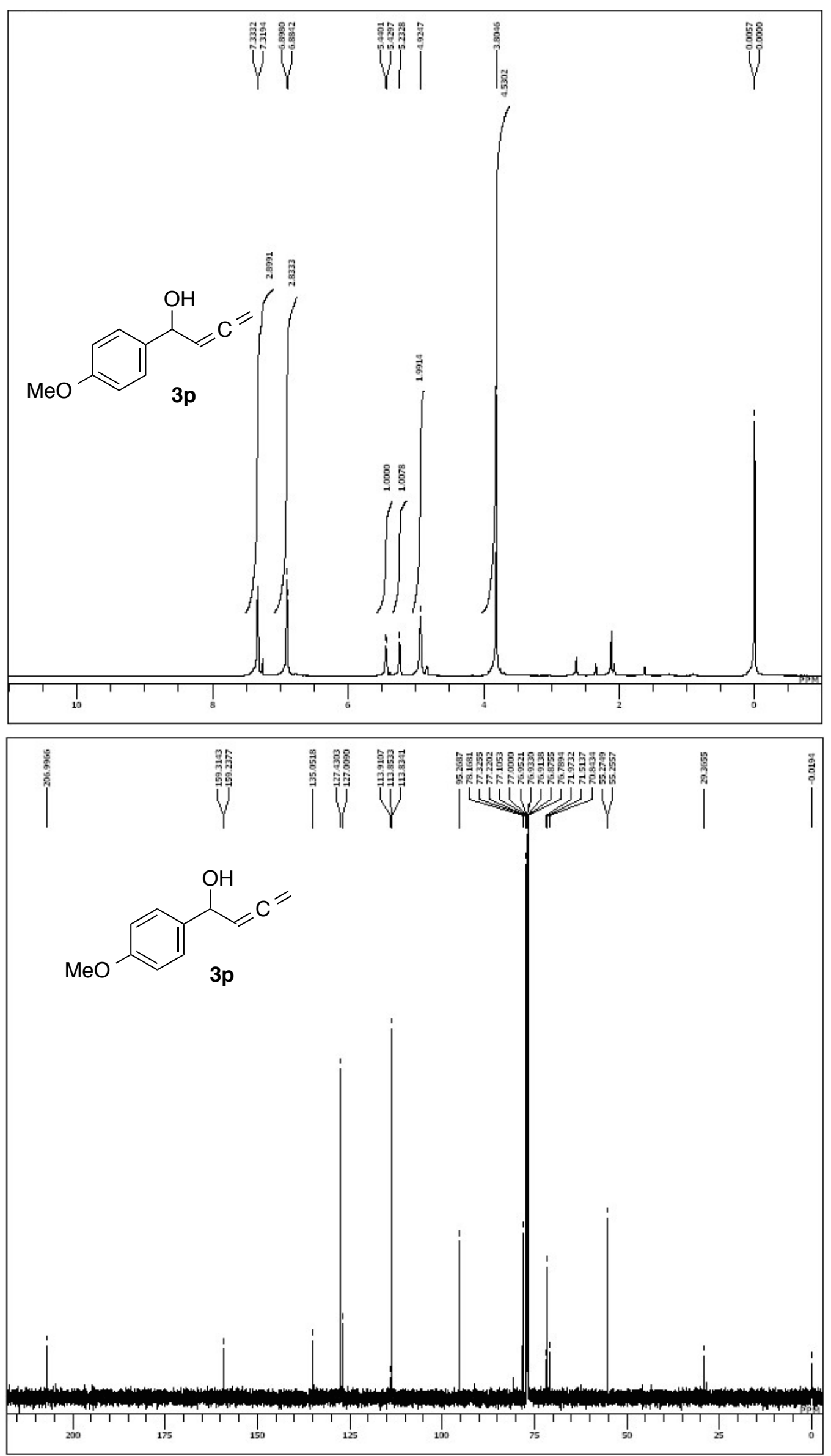

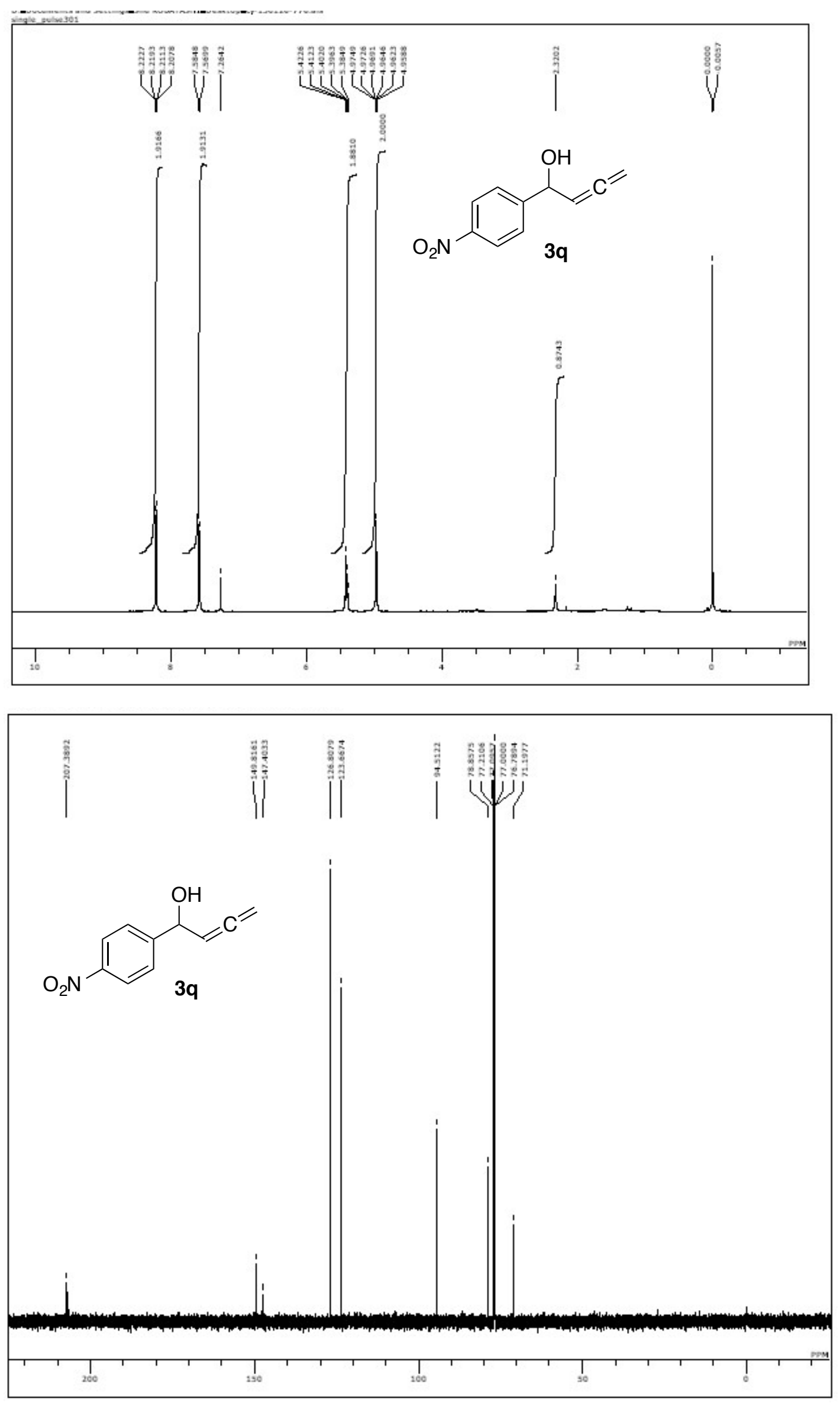

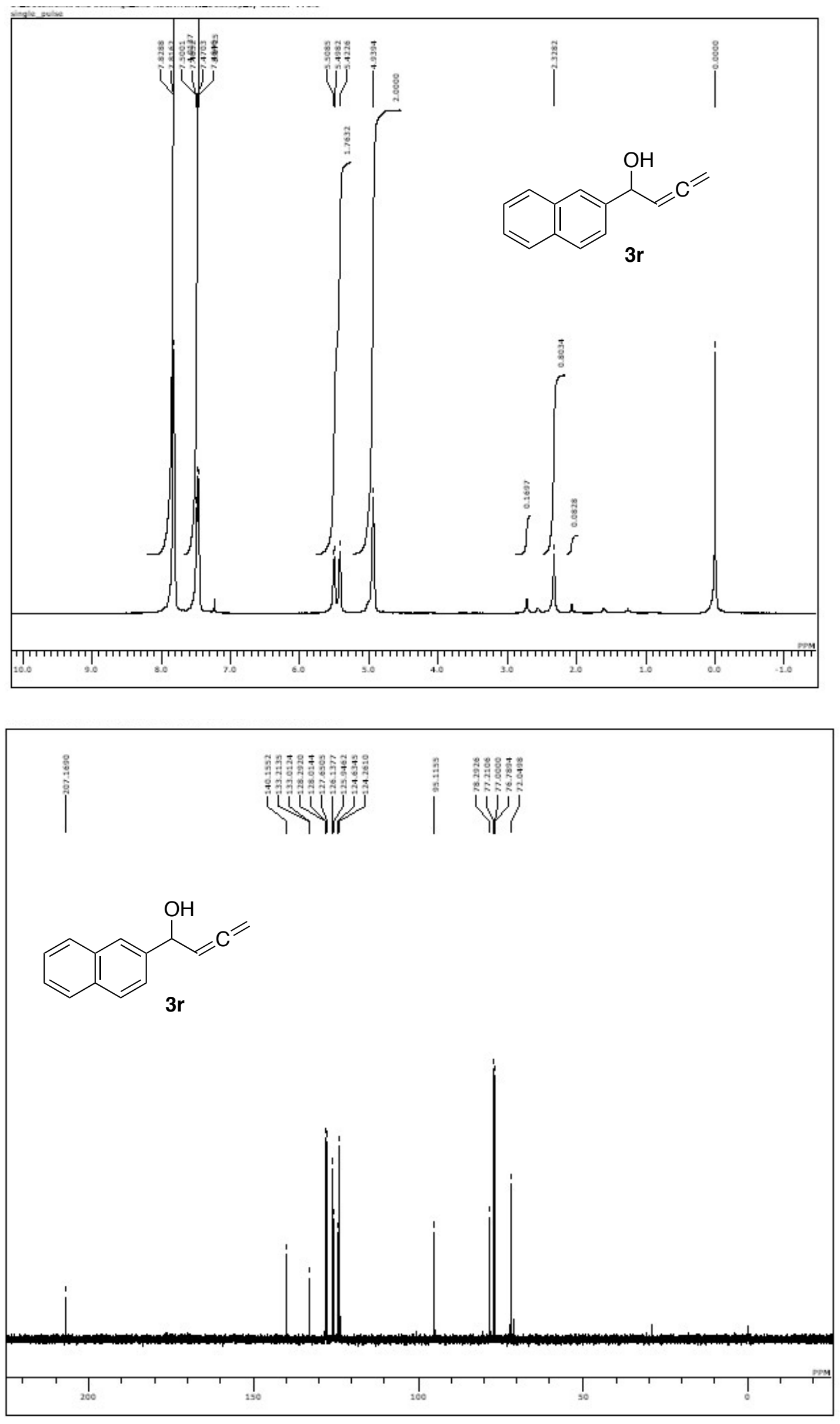

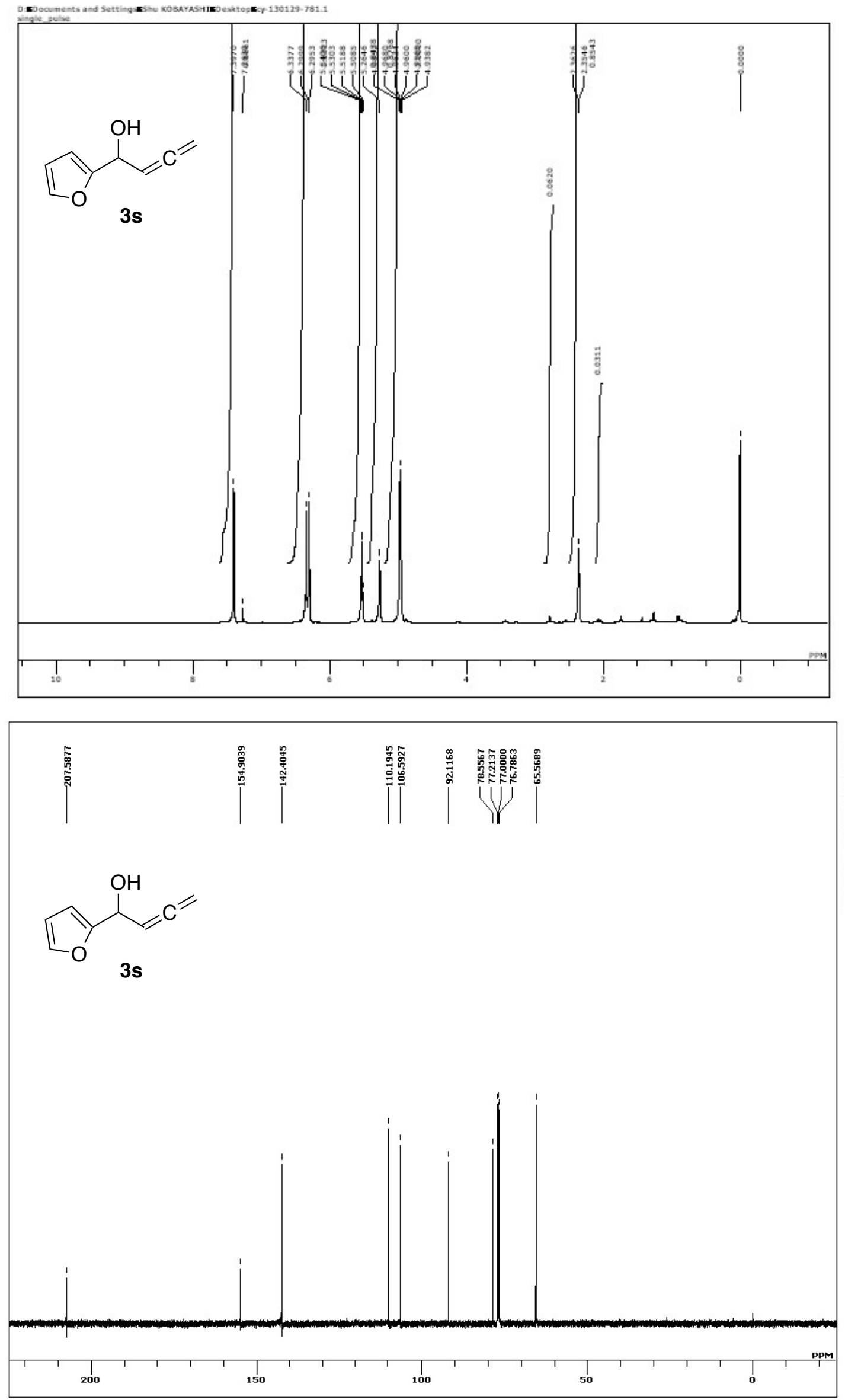

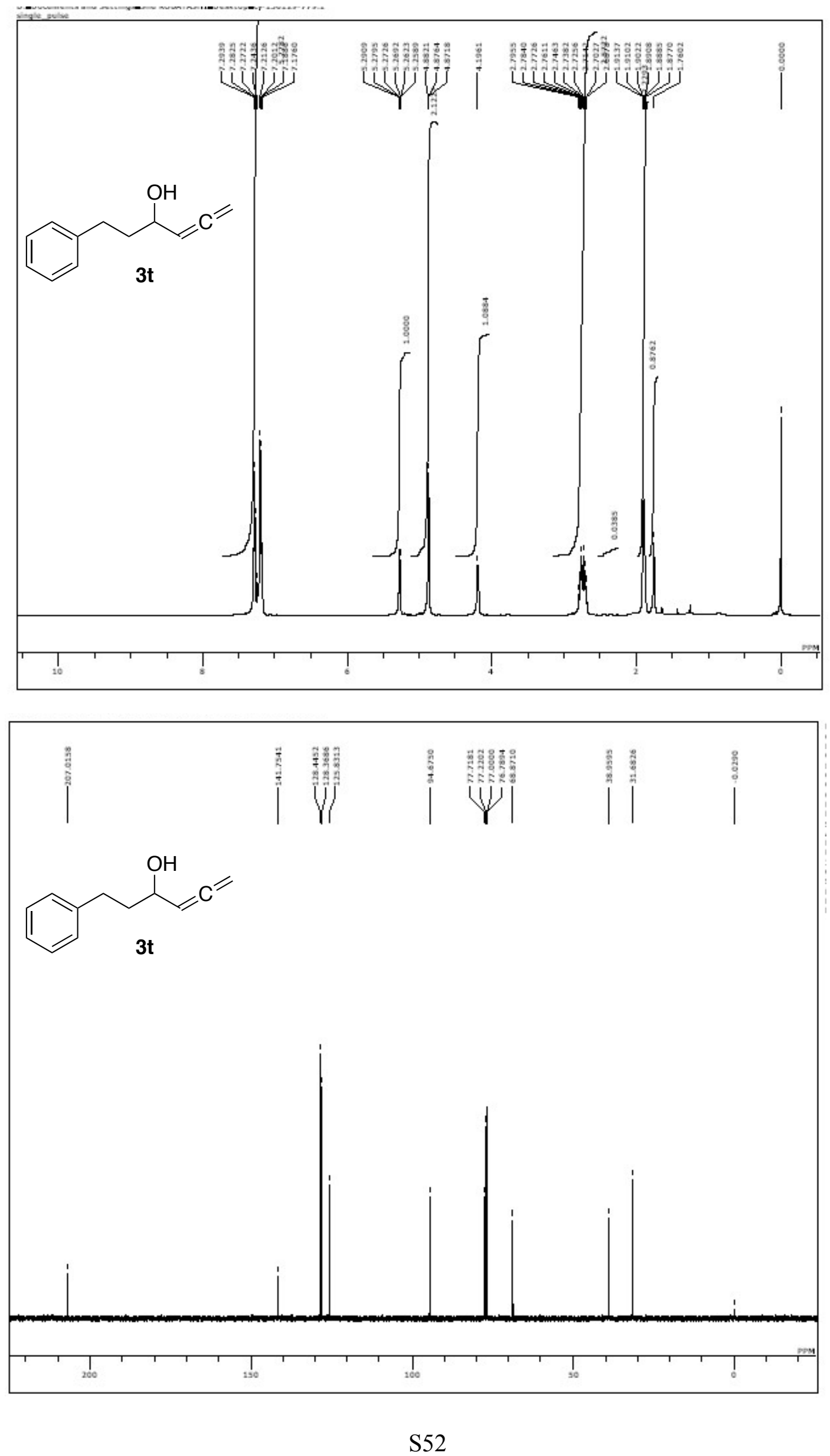

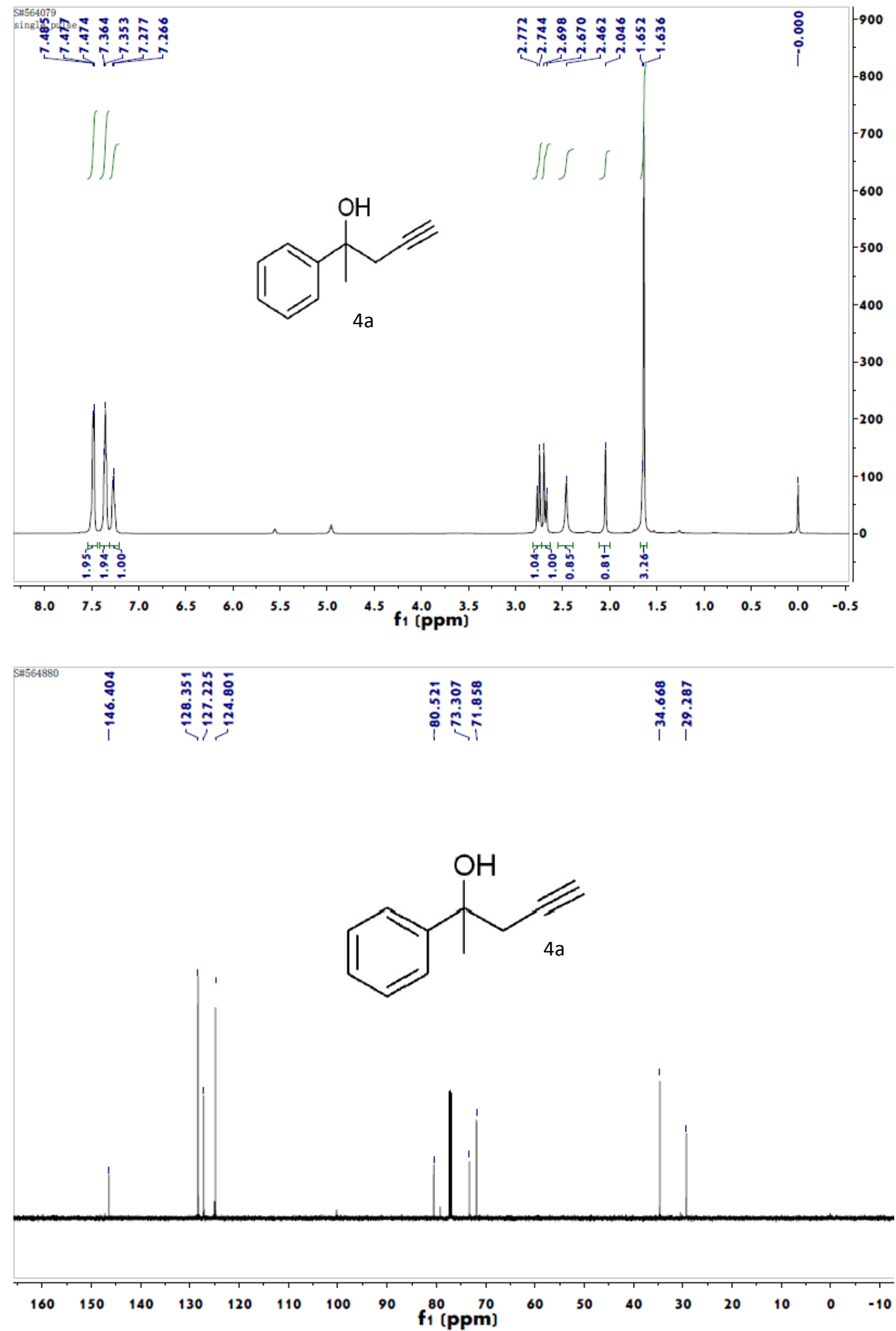

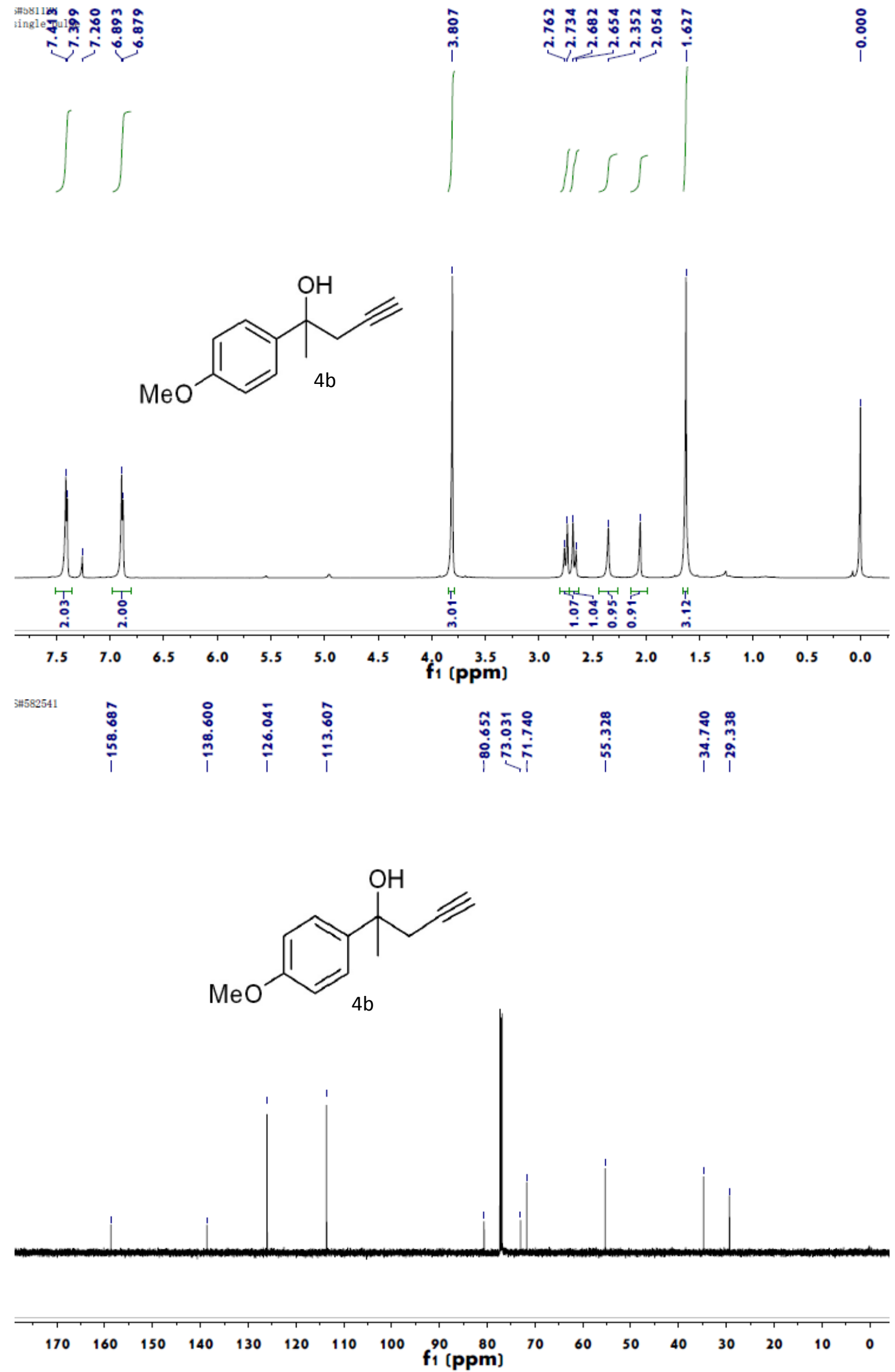

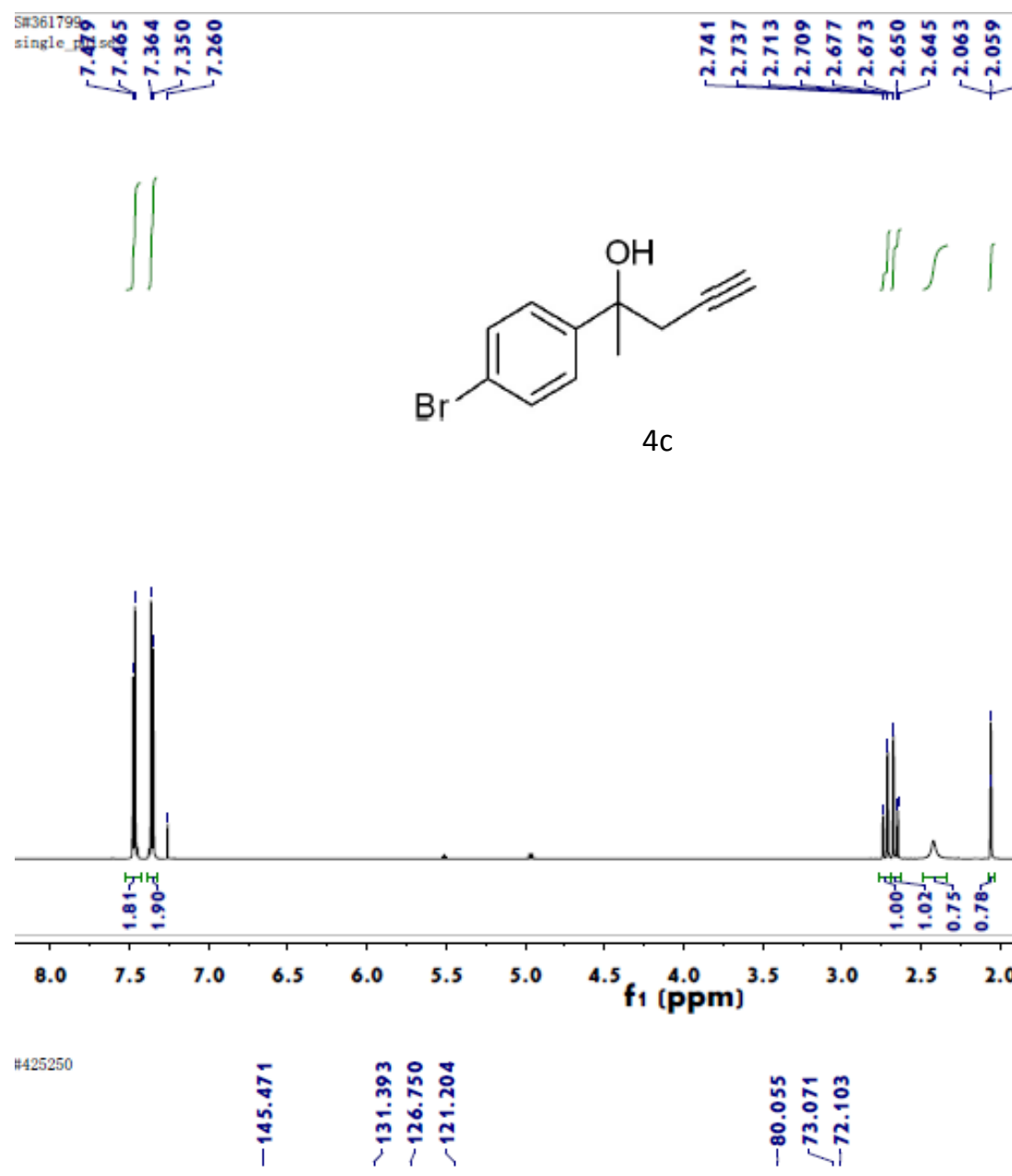<smiles>C#CCC(C)(O)c1ccc(Br)cc1</smiles>

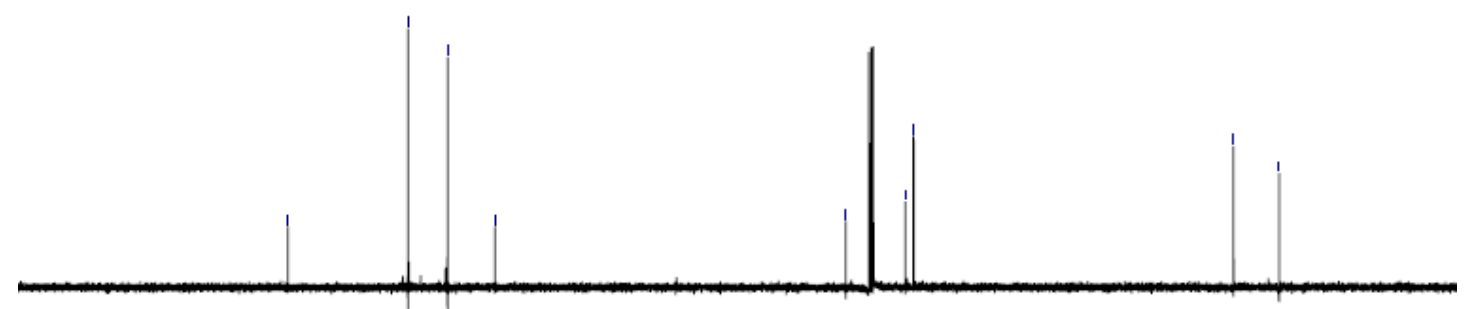

\begin{tabular}{|c|c|c|c|c|c|c|c|c|c|c|c|c|c|c|c|}
\hline 170 & 160 & 150 & 140 & 130 & 120 & 110 & $\begin{array}{l}100 \\
f_{1}\end{array}$ & $\begin{array}{c}90 \\
\text { ppm] }\end{array}$ & 80 & 70 & 60 & 50 & 40 & 30 & 20 \\
\hline
\end{tabular}


single

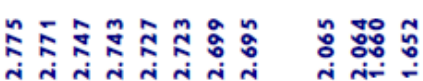

乎更

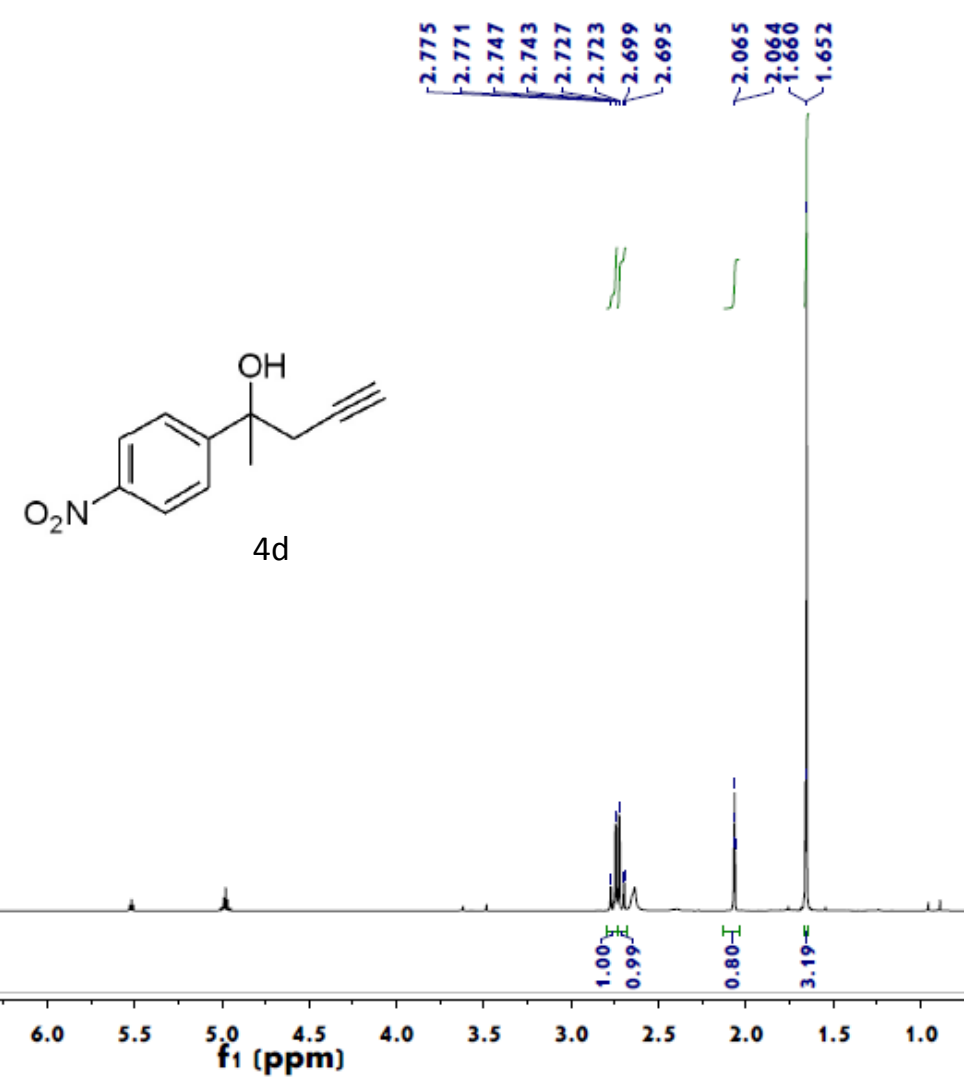

"375557

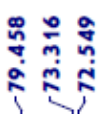

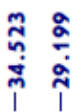
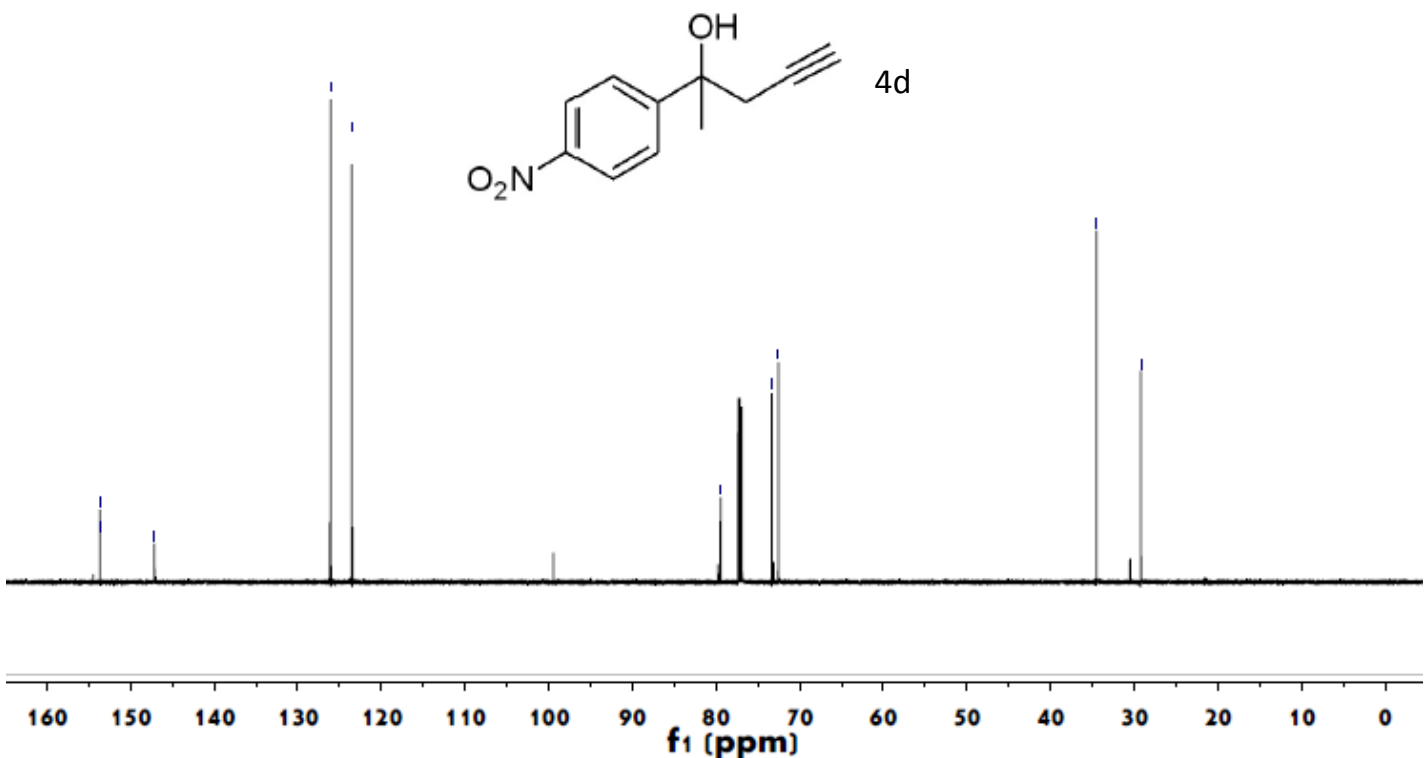
<smiles>C#CCC(C)(O)c1ccccc1C</smiles>
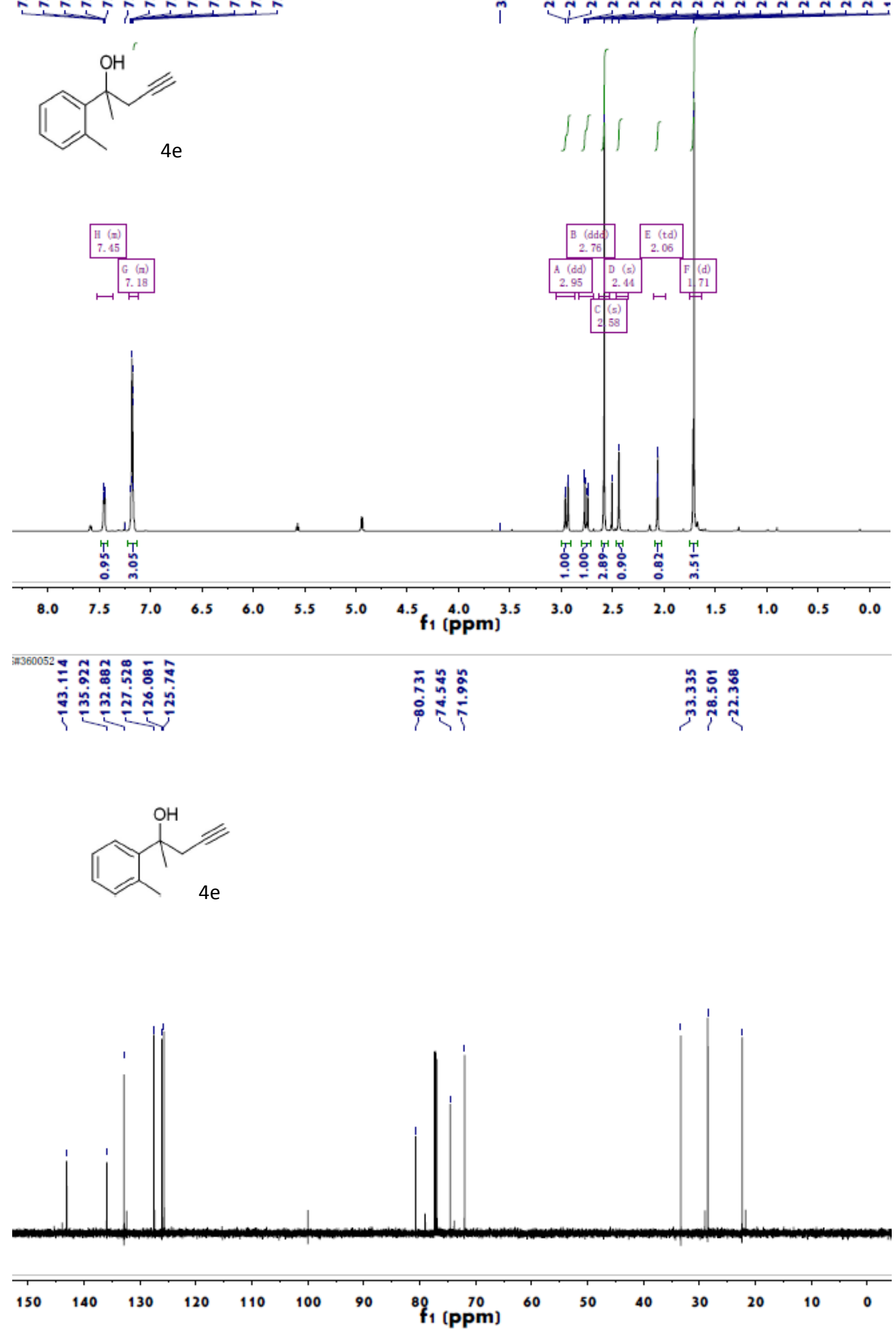

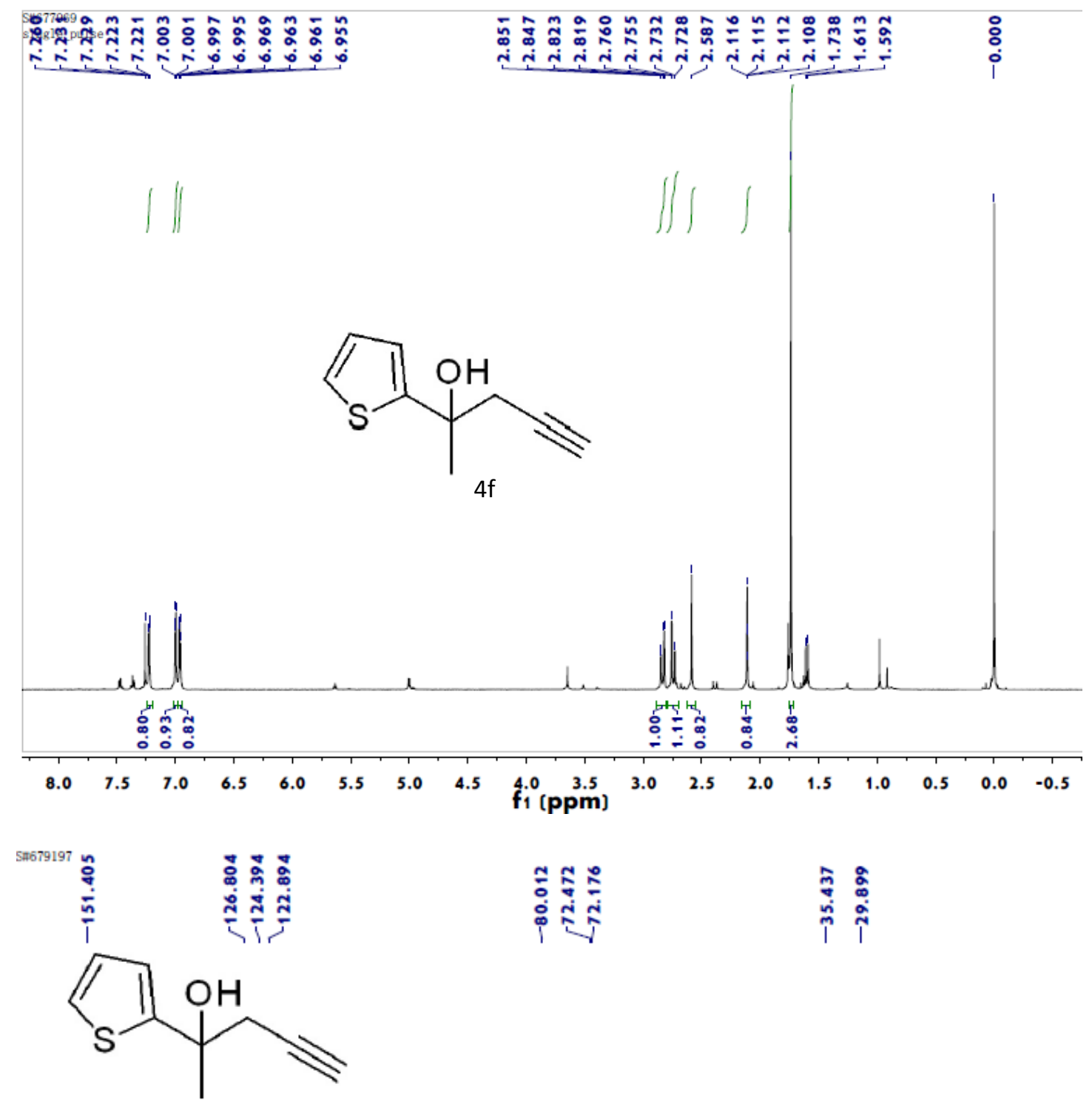

$4 f$

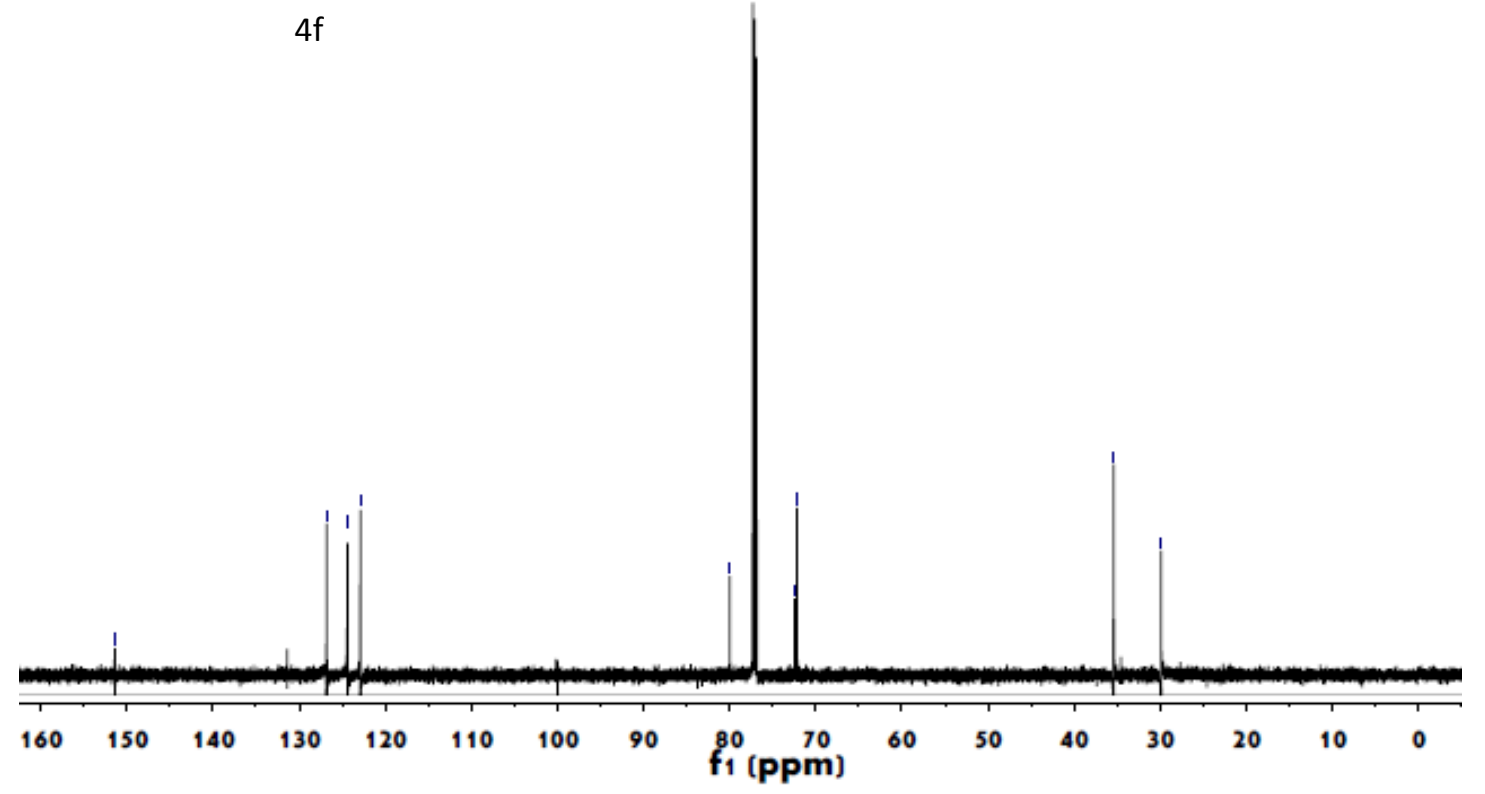




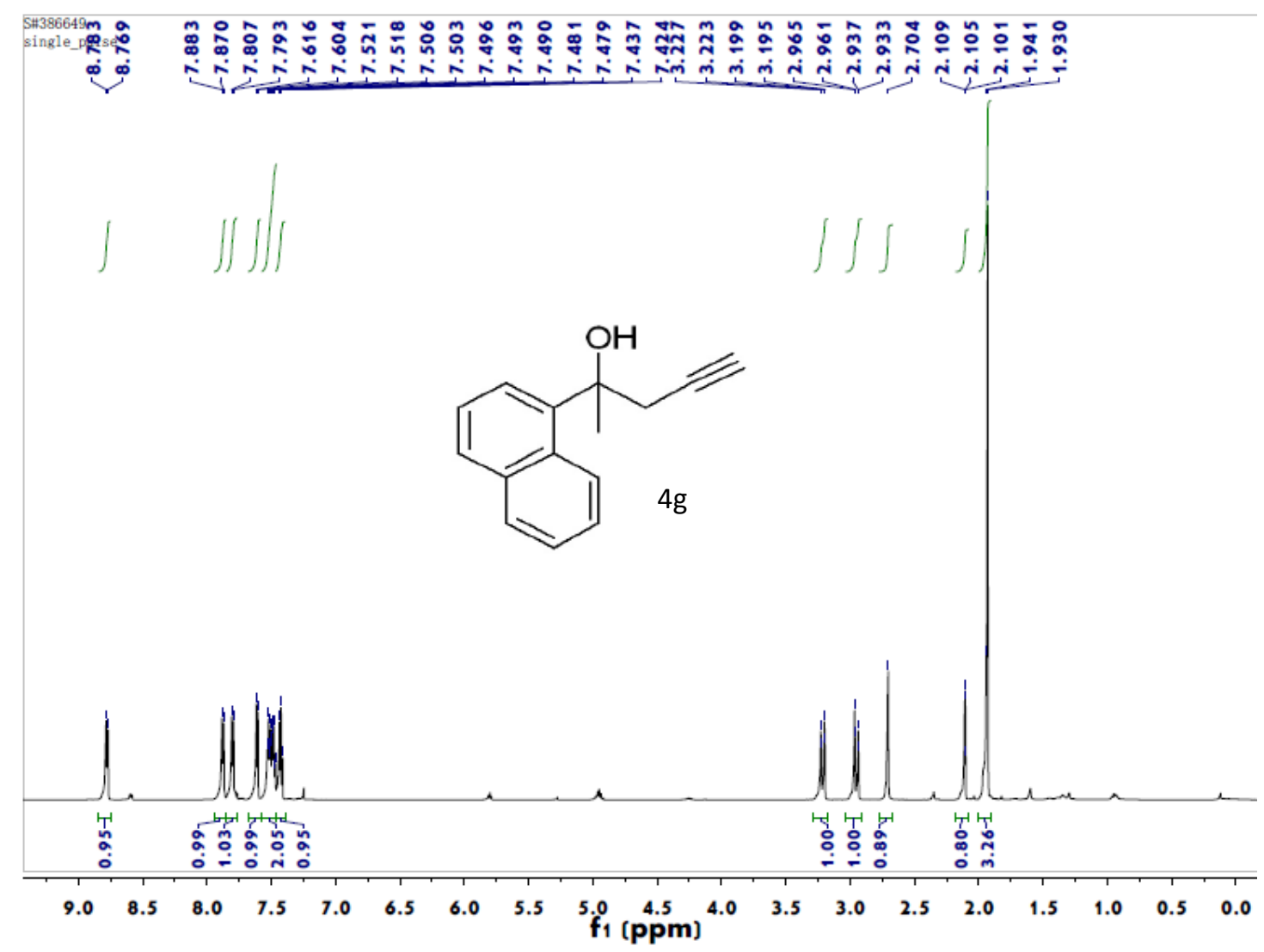

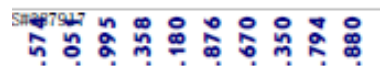

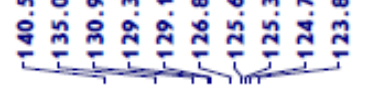

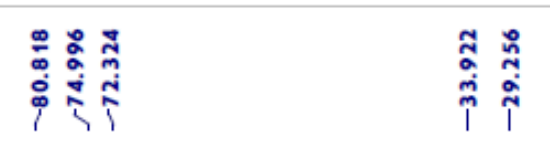<smiles>C#CCC(C)(O)c1cccc2ccccc12</smiles>

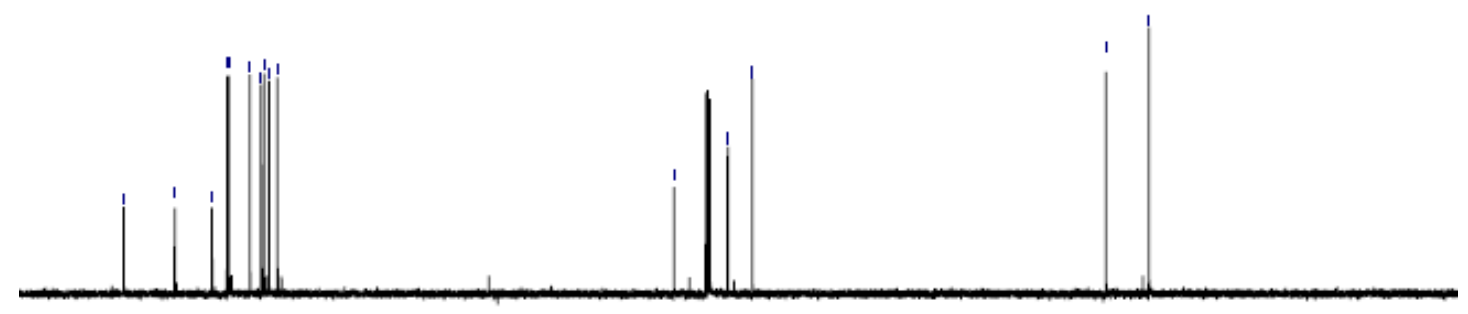

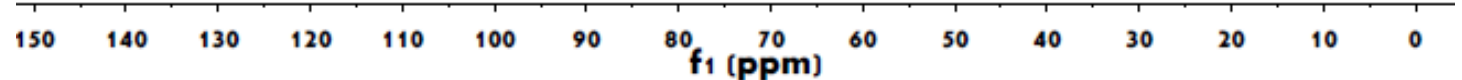




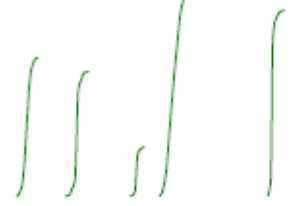<smiles>C#CCC(C)(O)CCc1ccccc1</smiles>

$4 \mathrm{~h}$
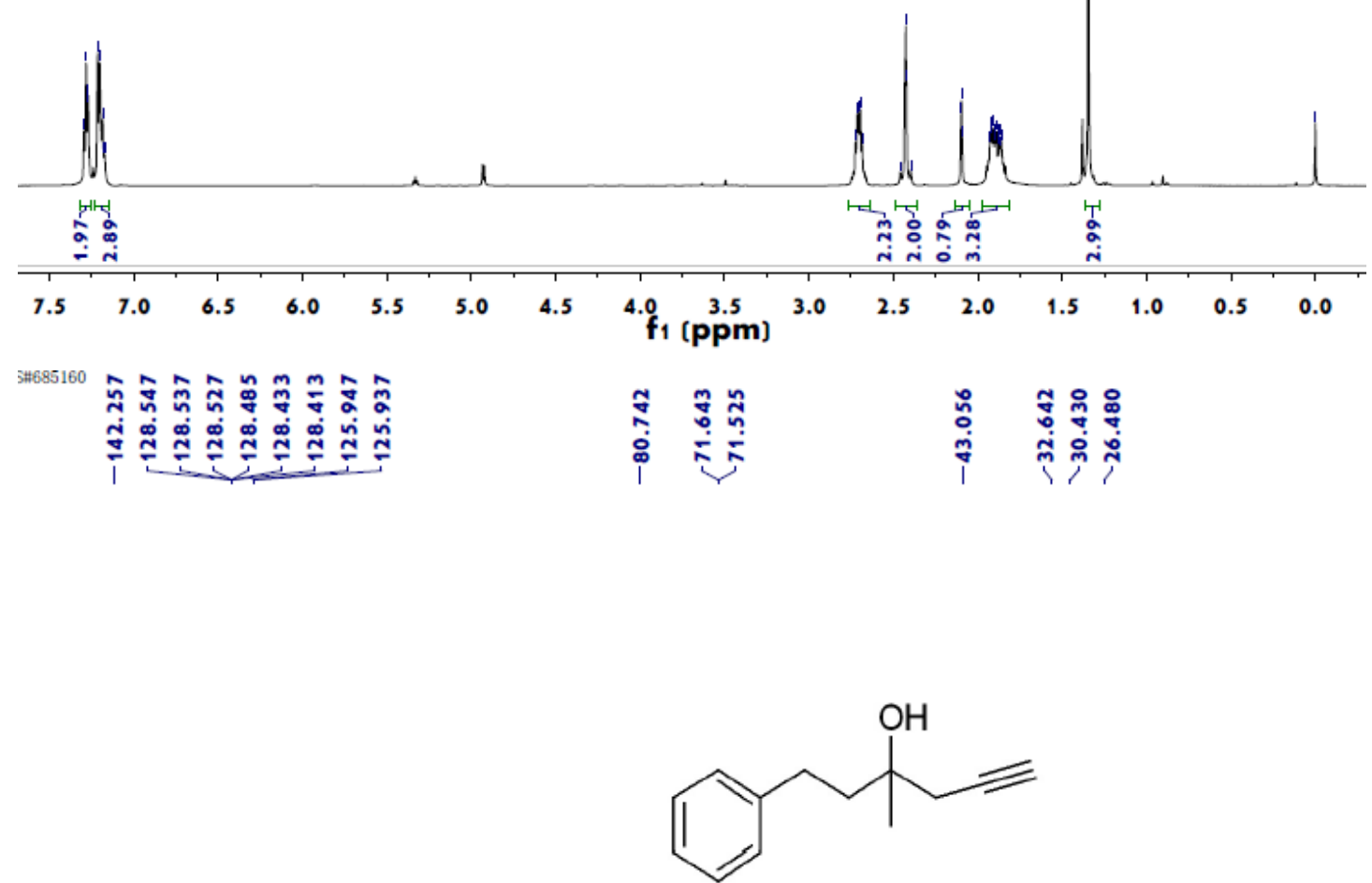

$4 h$

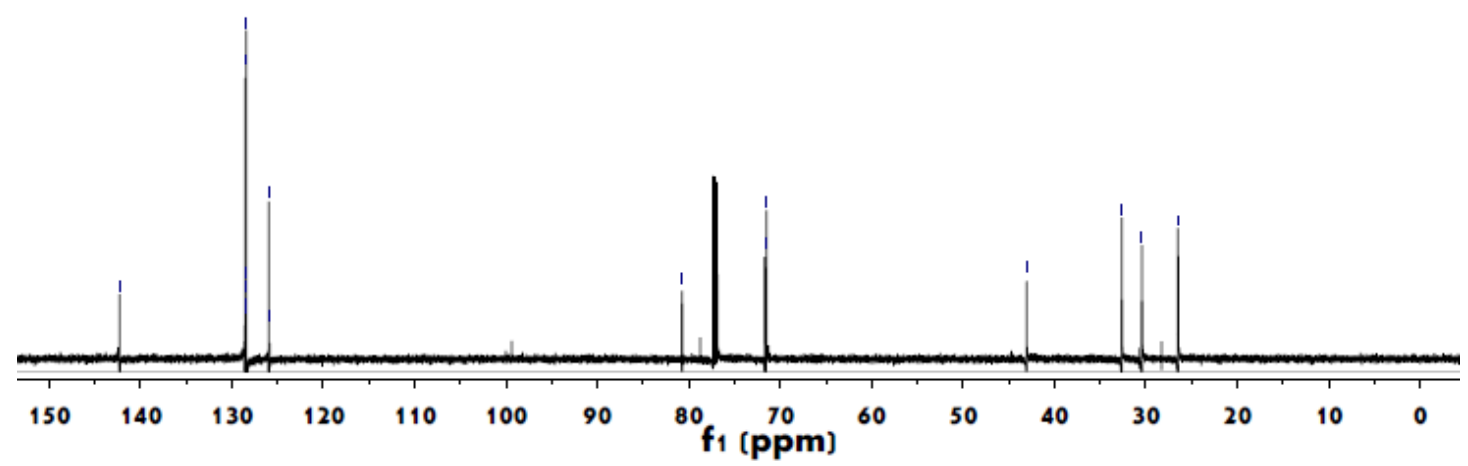



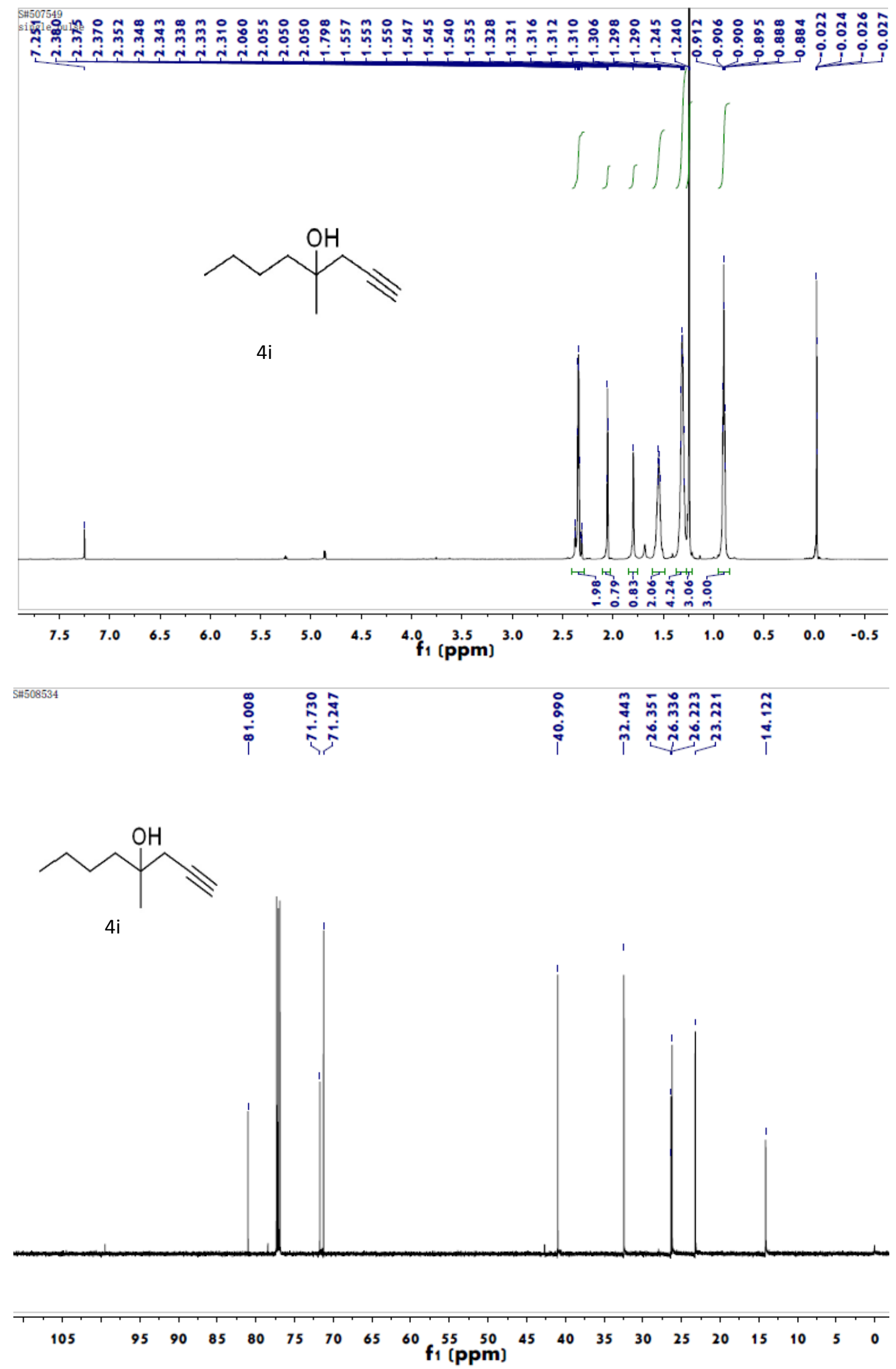

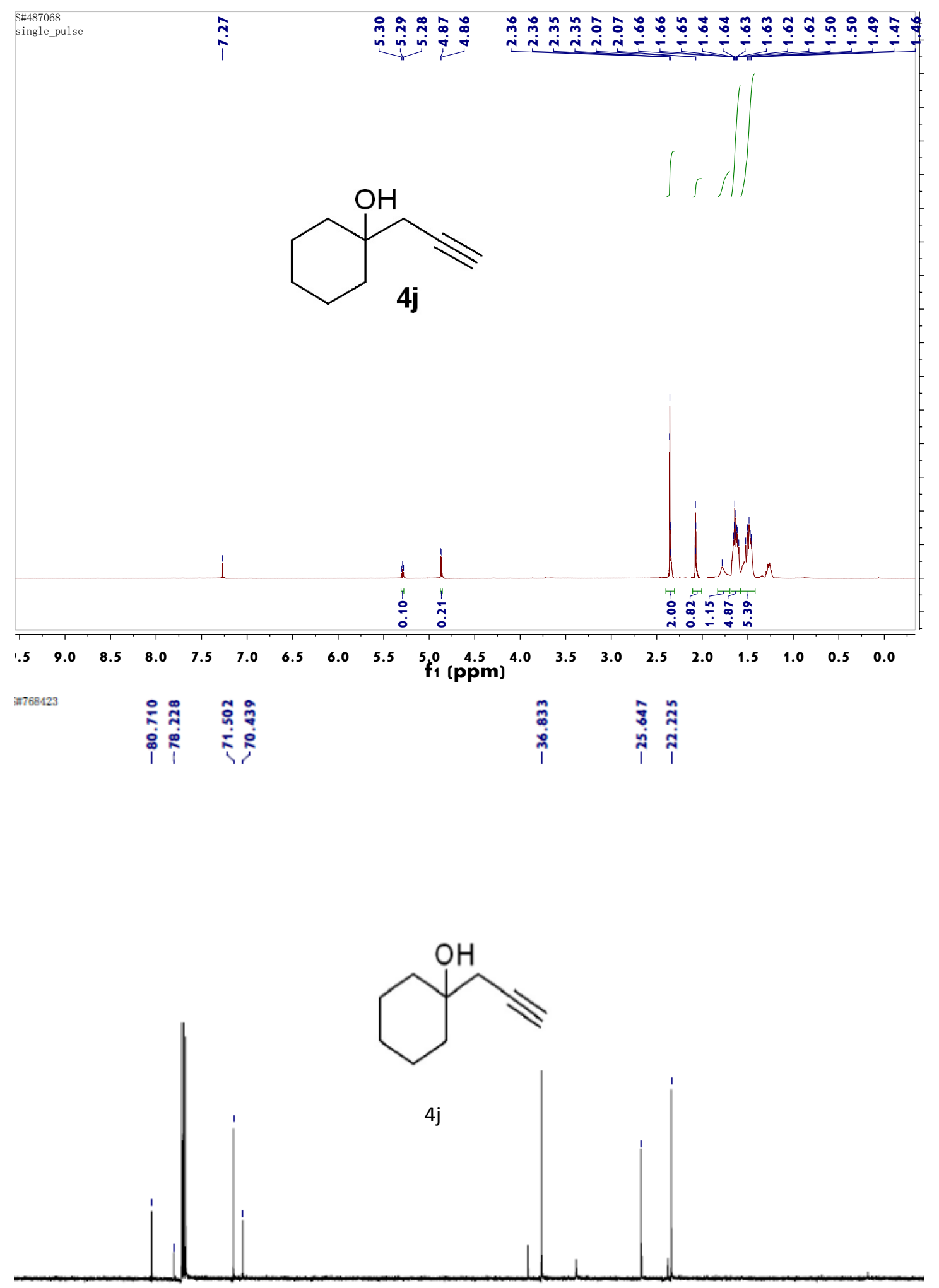

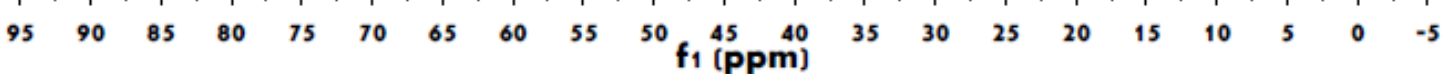



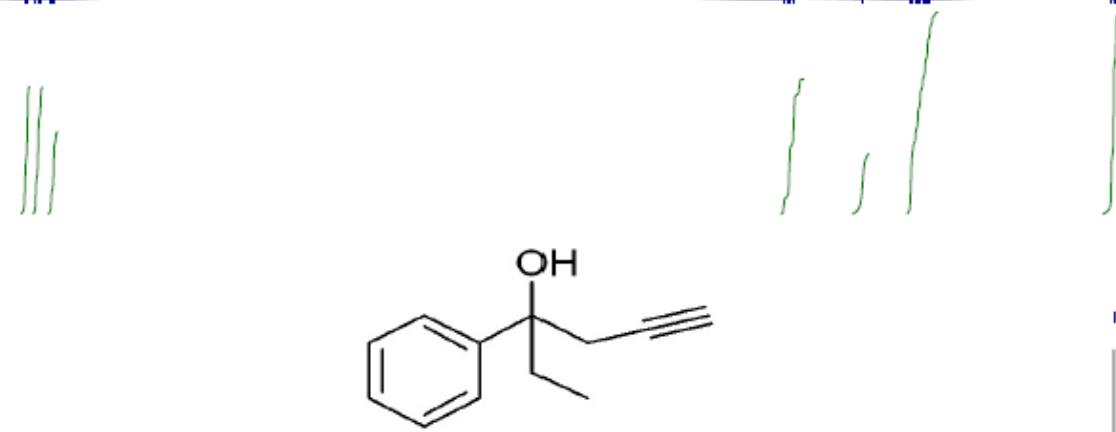

$4 \mathrm{k}$

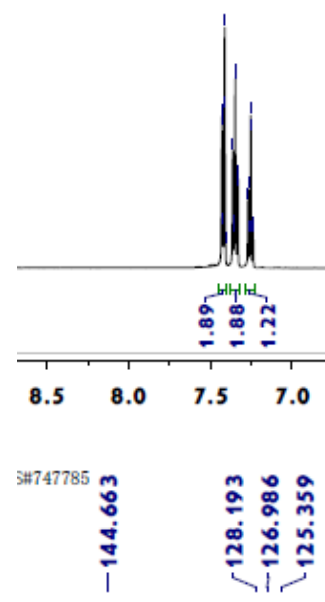

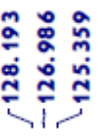

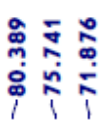

ฟั

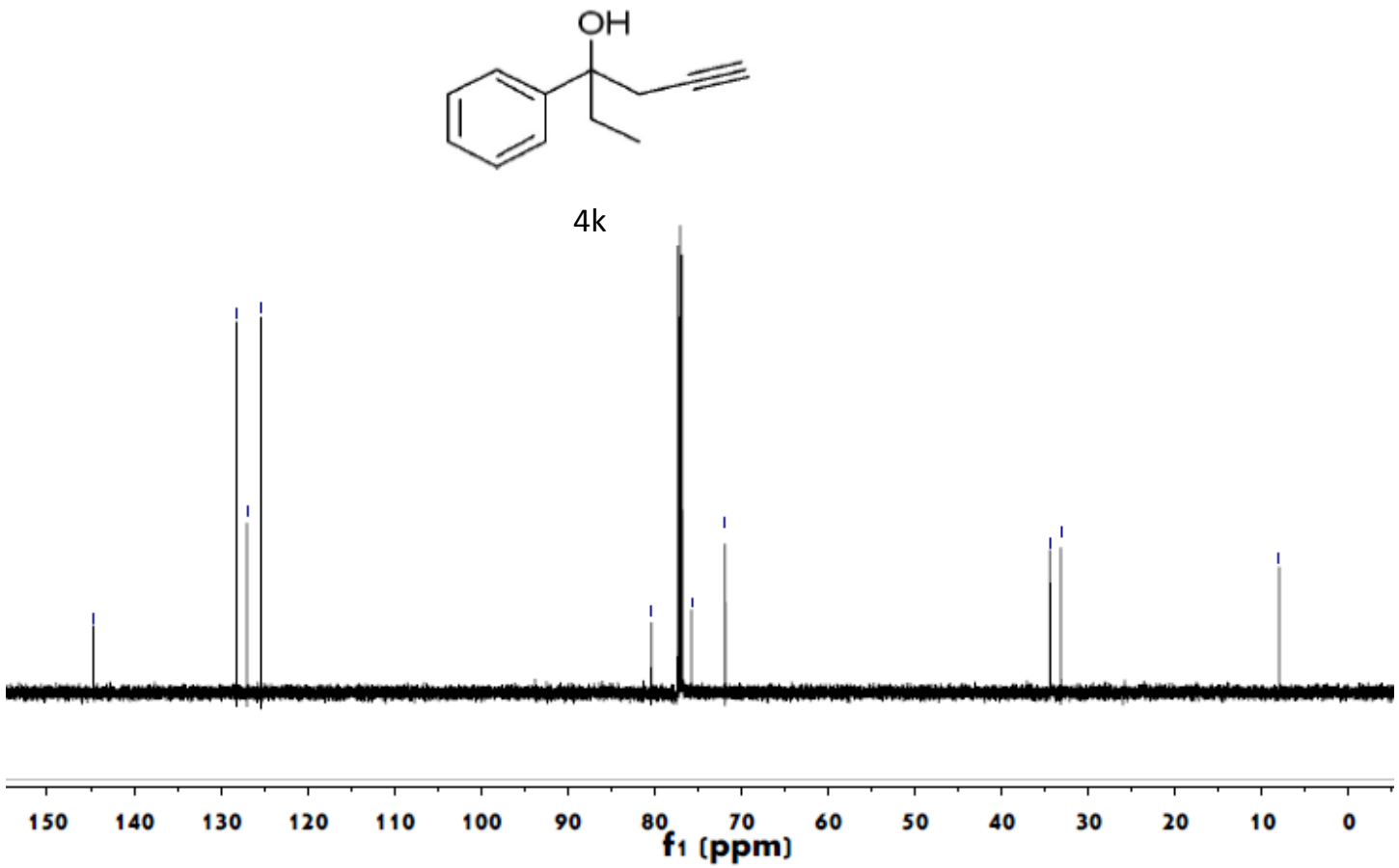

$4 \mathrm{k}$
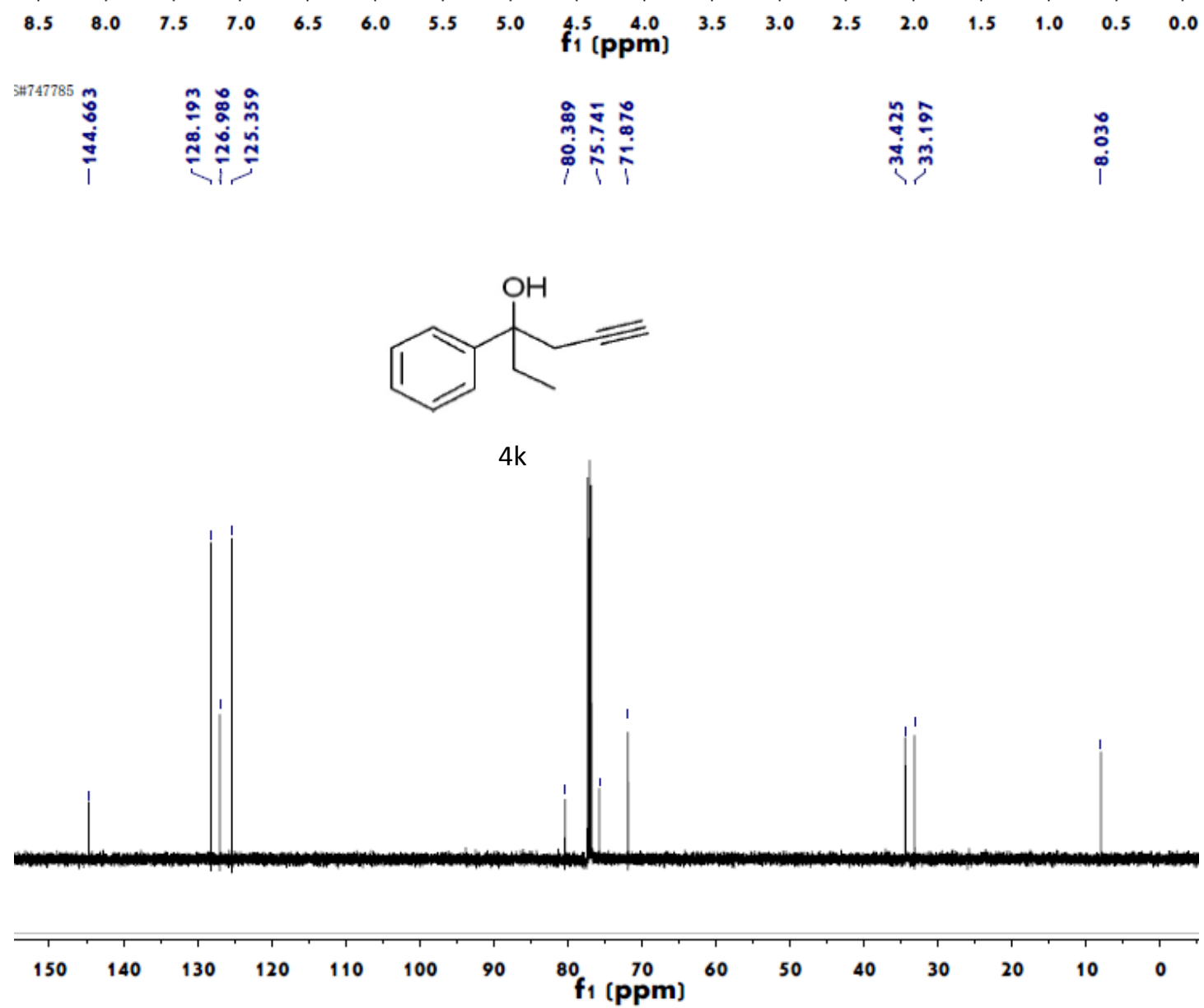


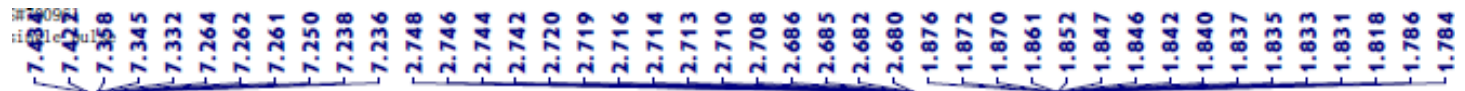
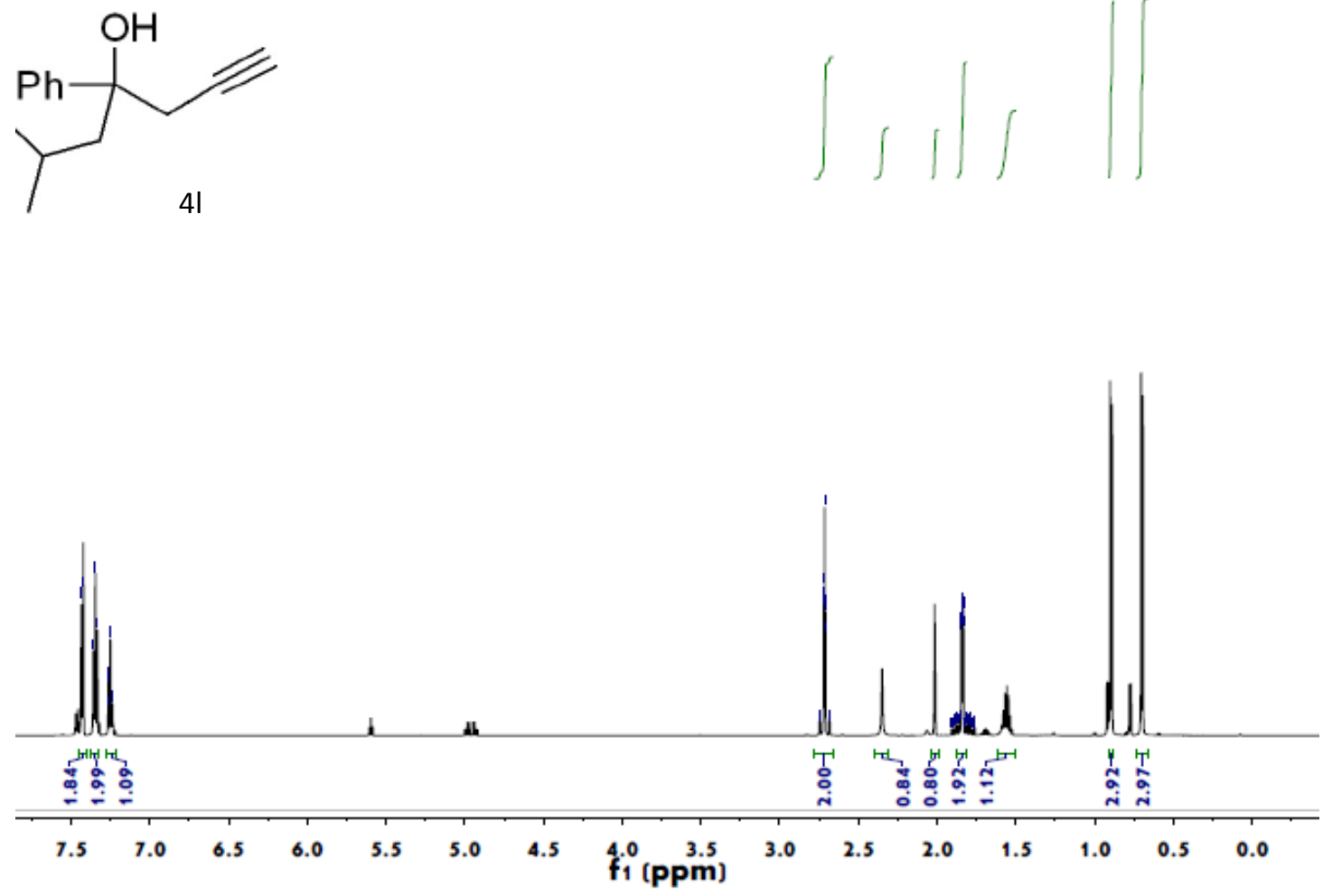

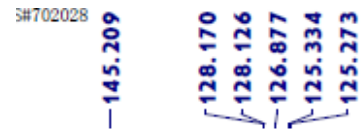

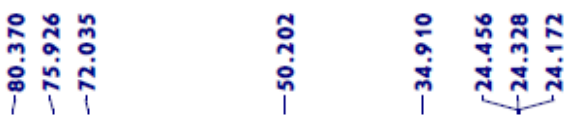
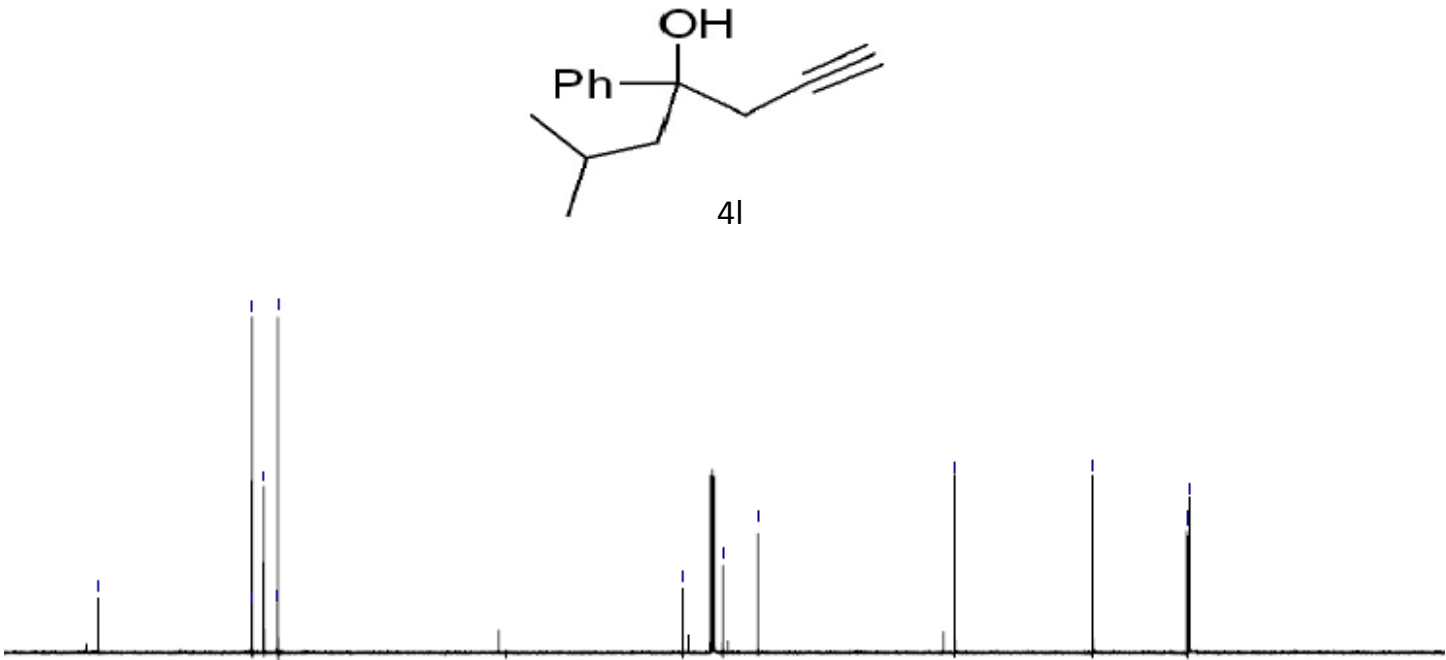

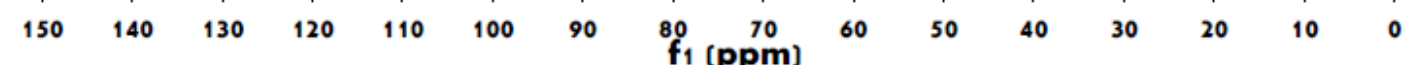




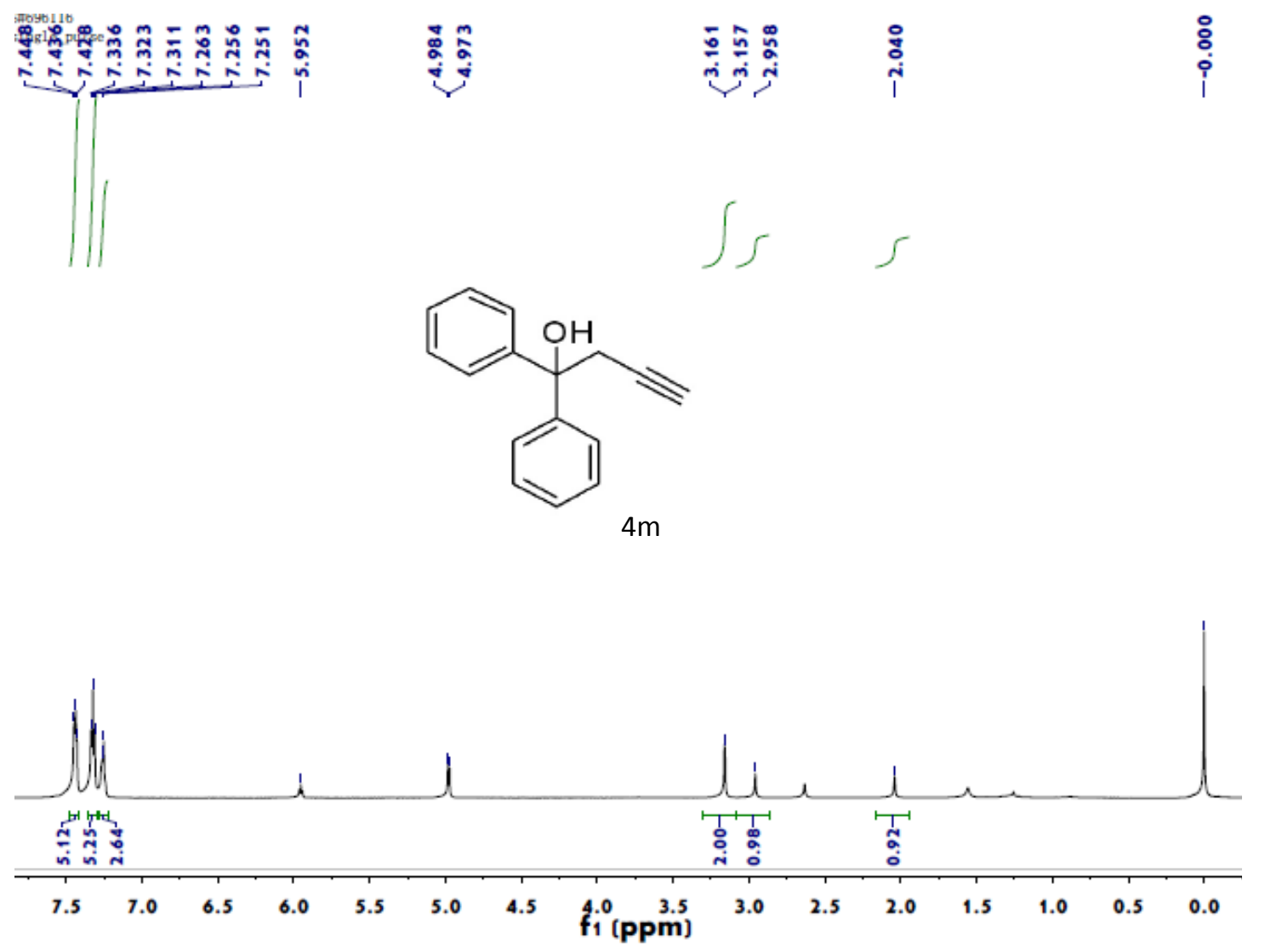

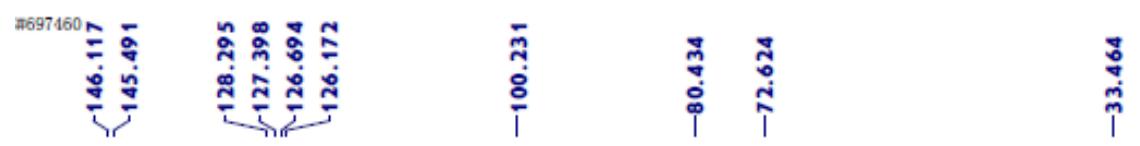

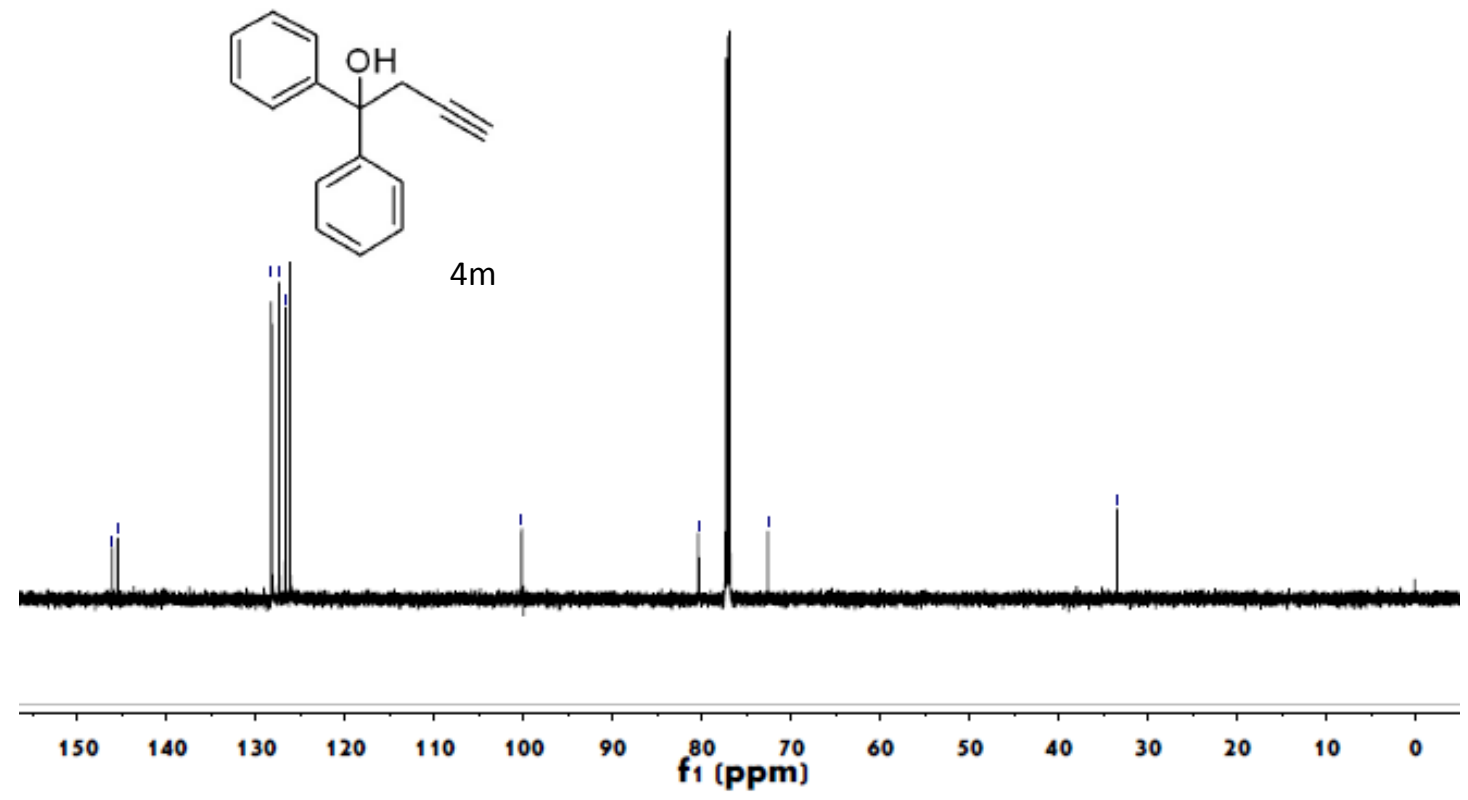



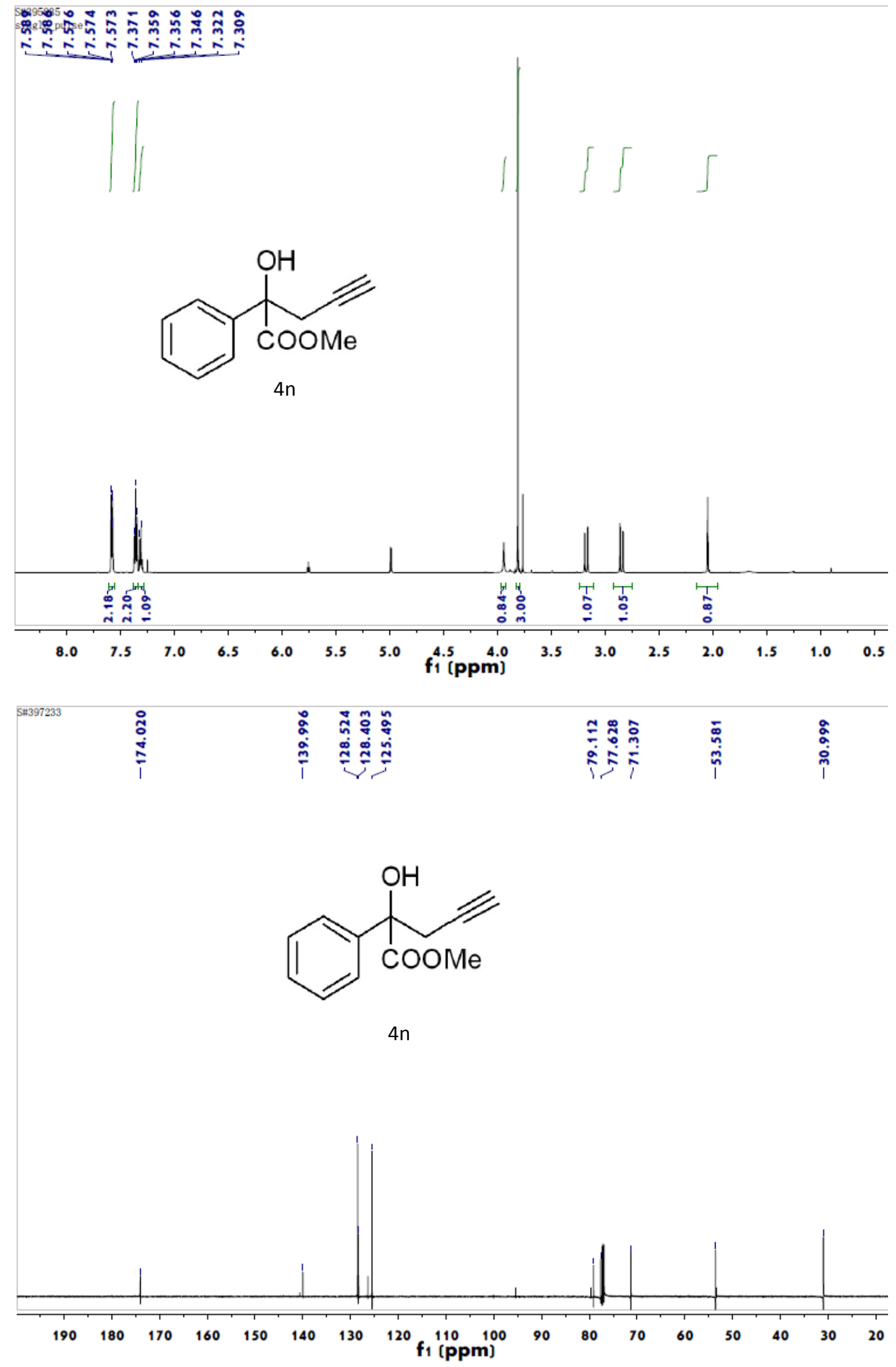

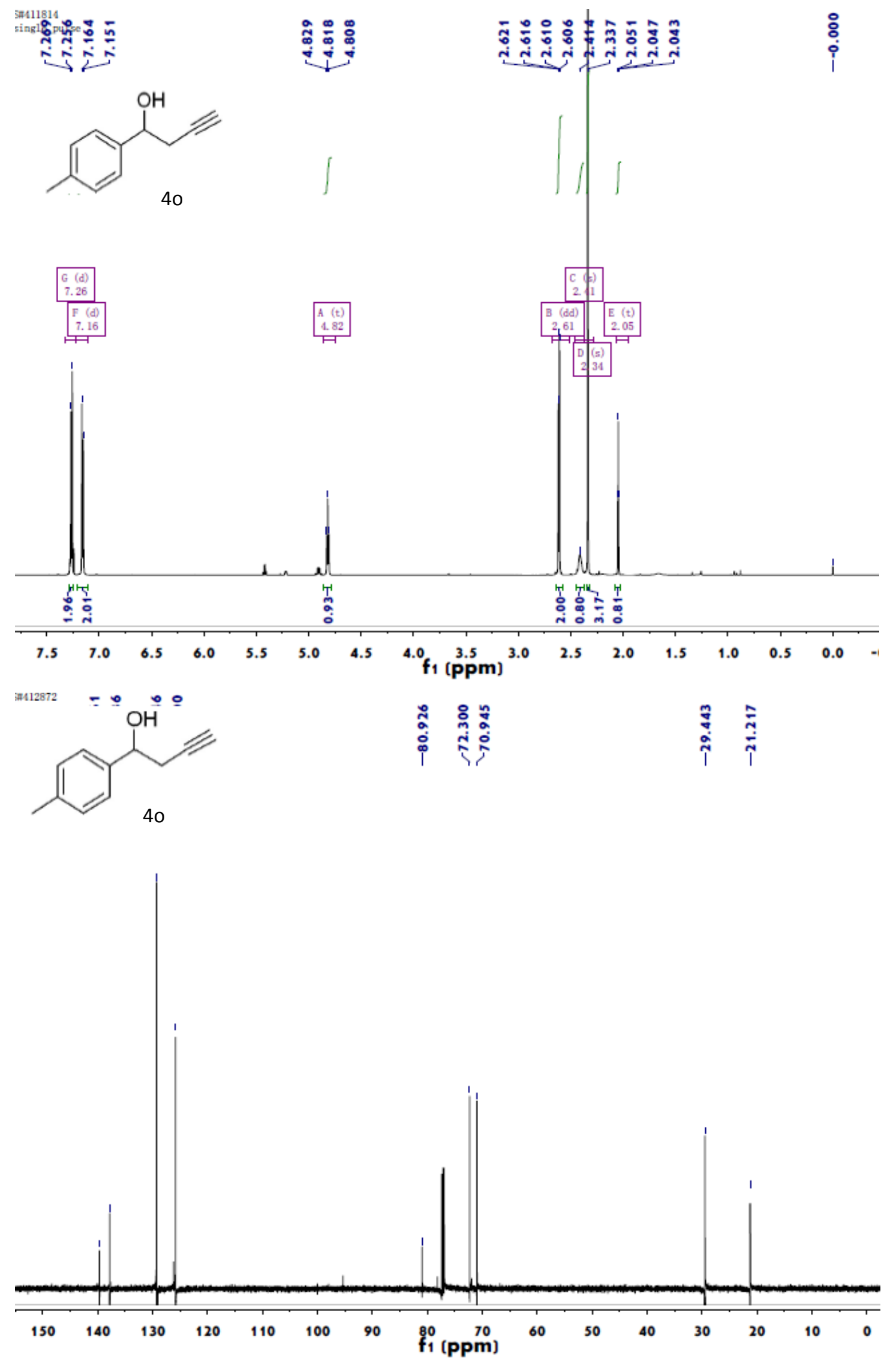


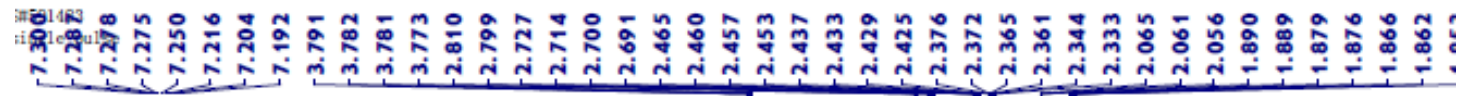<smiles>C#CCC(O)CCc1ccccc1</smiles>
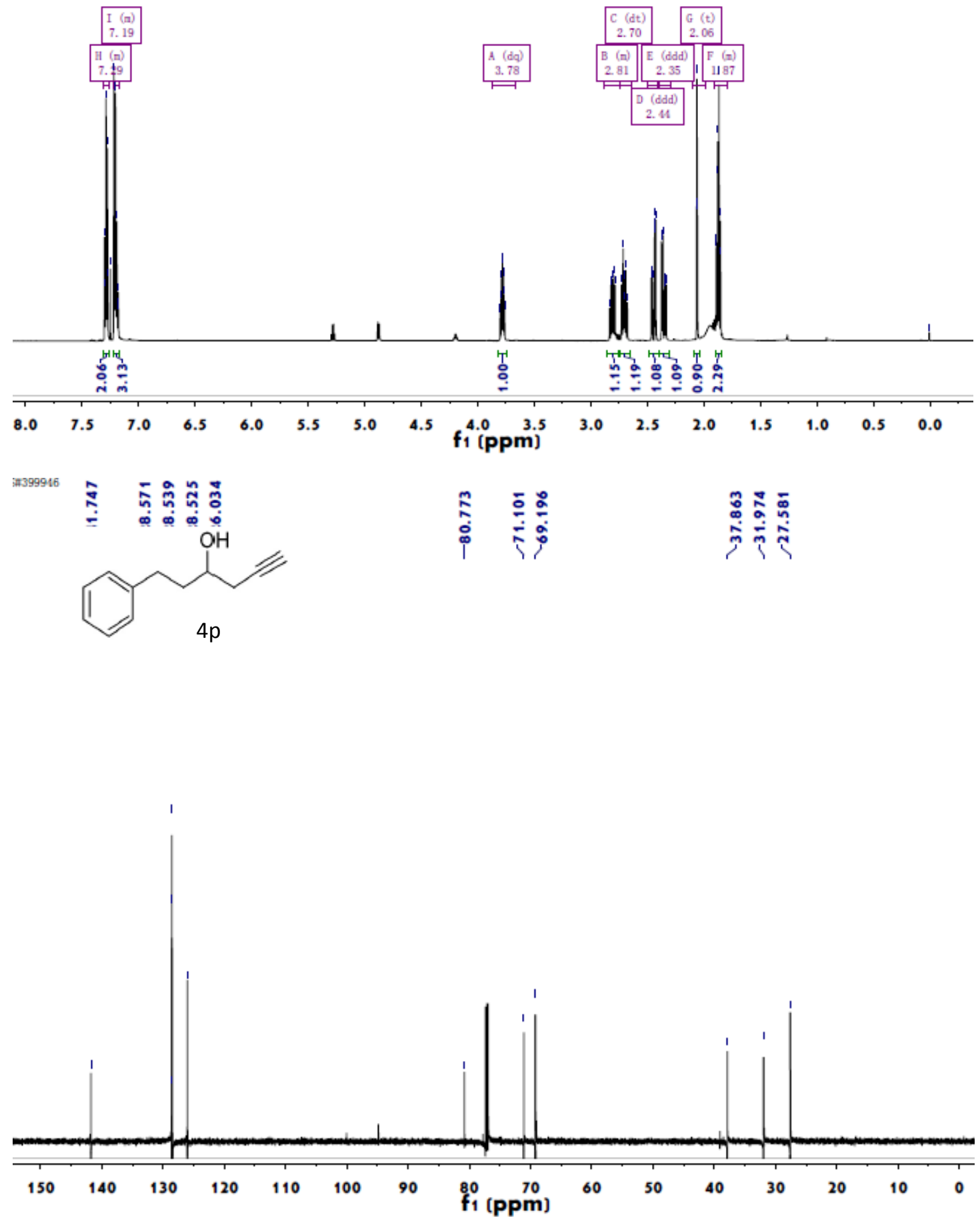

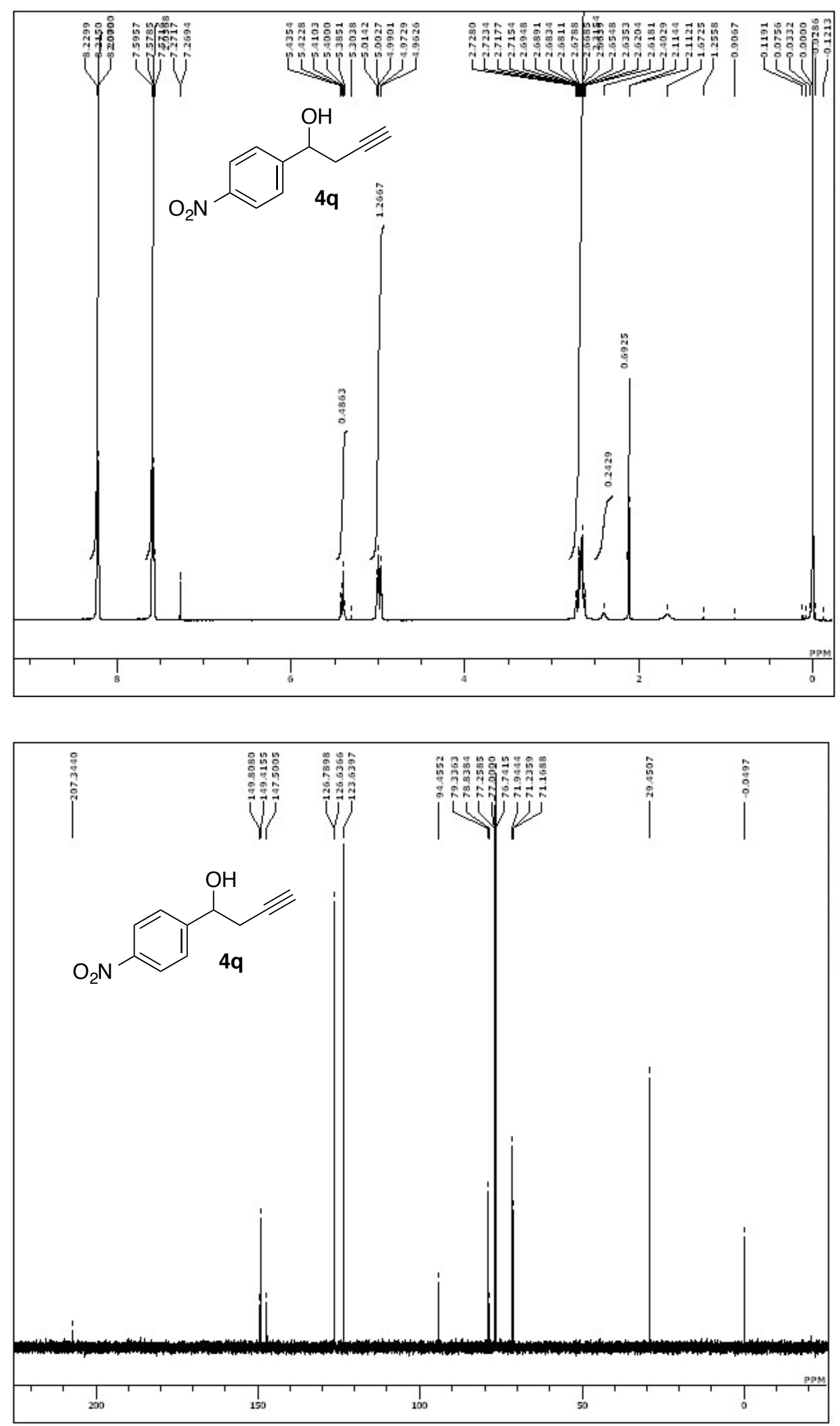


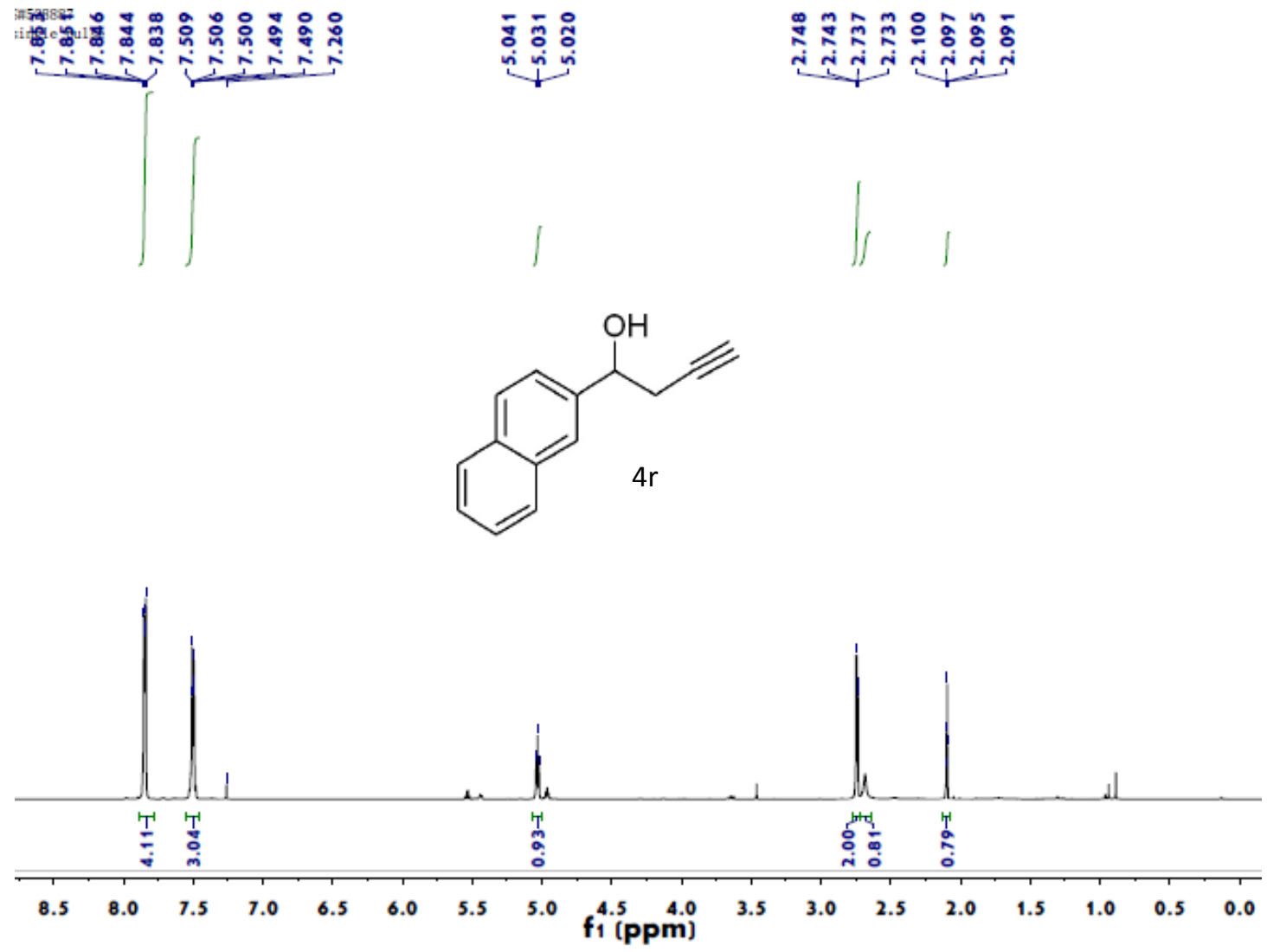

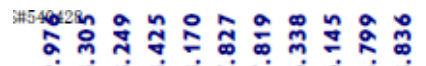

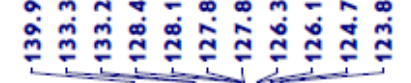

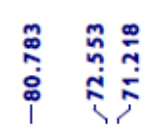

ฺั<smiles>C#CCC(O)c1ccc2ccccc2c1</smiles>

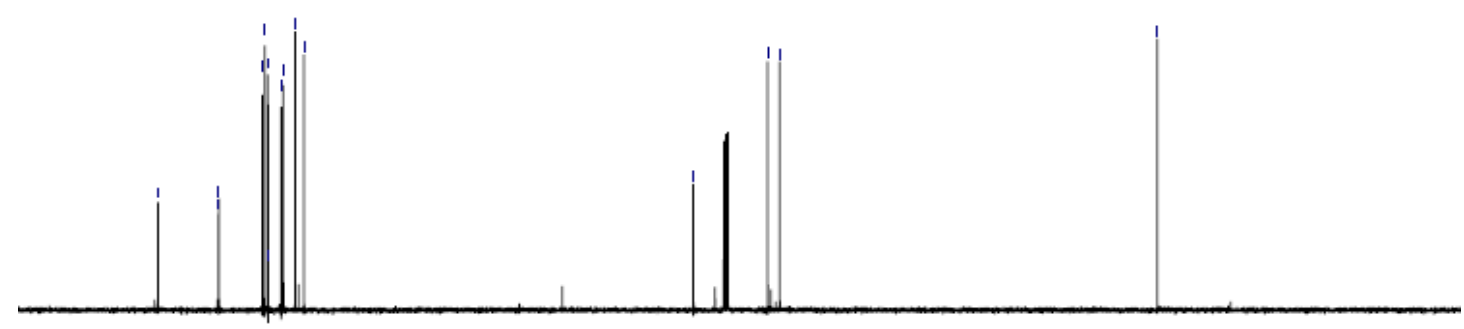

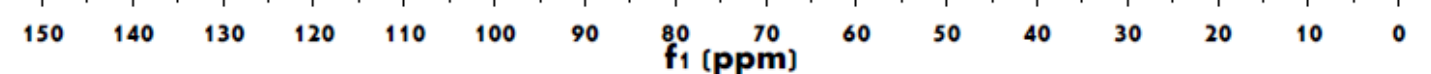



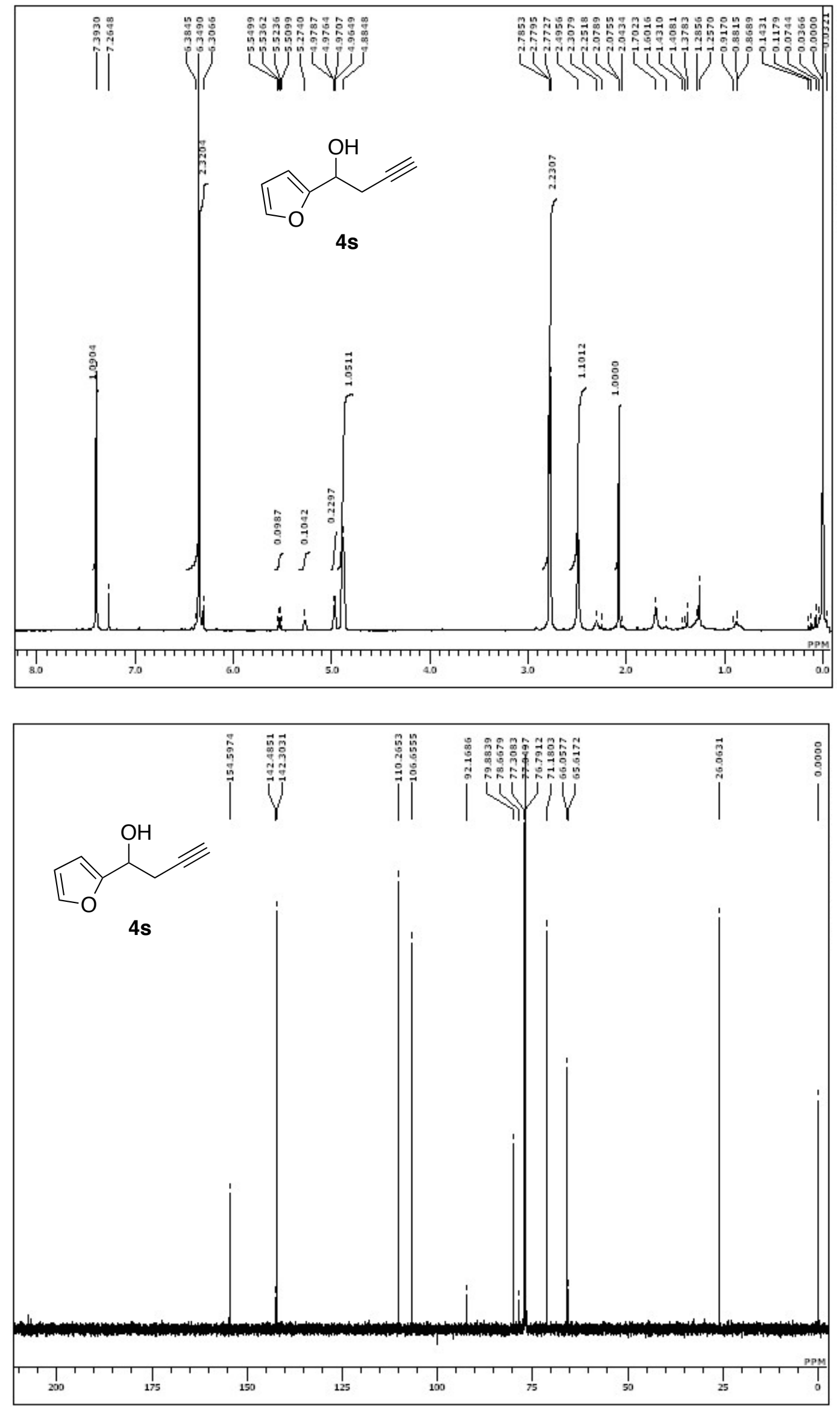


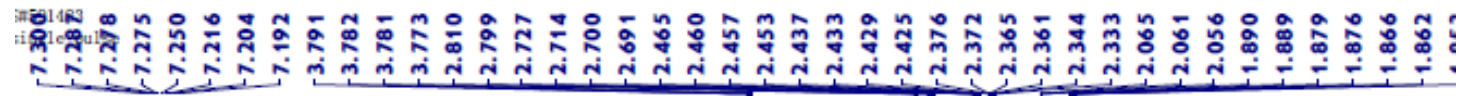<smiles>C#CCC(O)CCc1ccccc1</smiles><smiles>C=C1C=CC=C1</smiles>
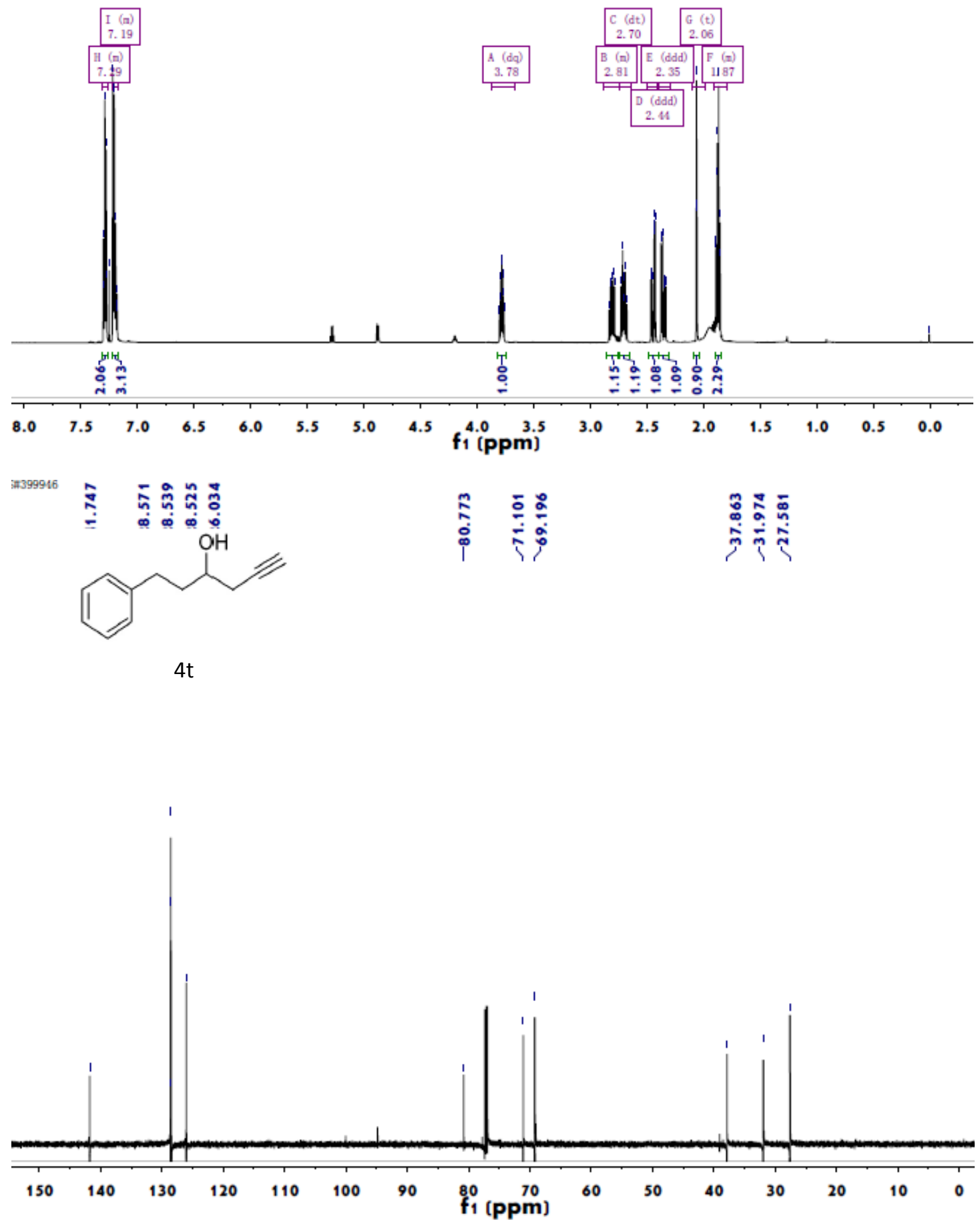


\section{HPLC Charts of 3n}<smiles>C=C=CC(O)(C(C)=O)c1ccccc1</smiles>

\section{Racemic 3n}

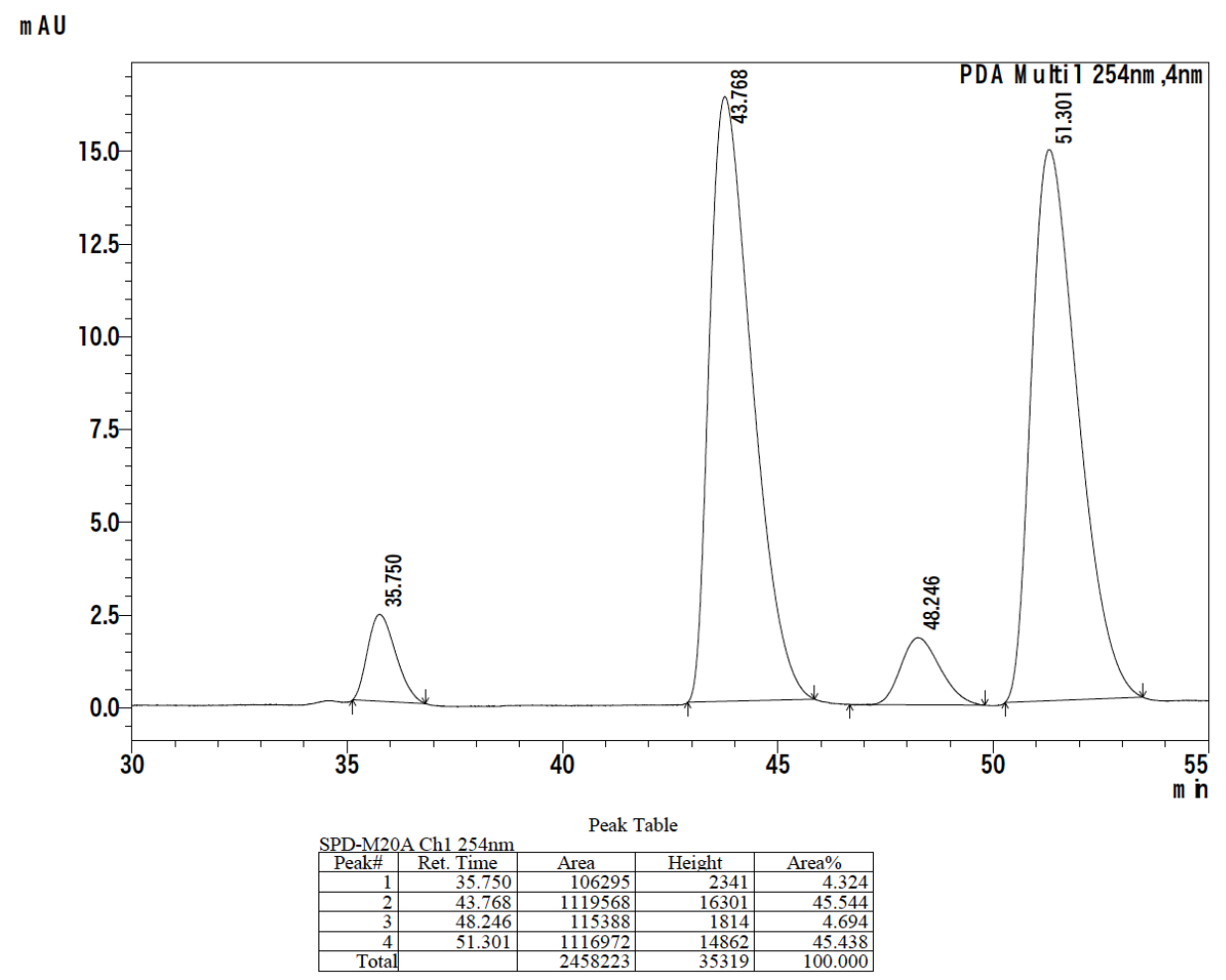

Optically active $\mathbf{3 n}$

mAU

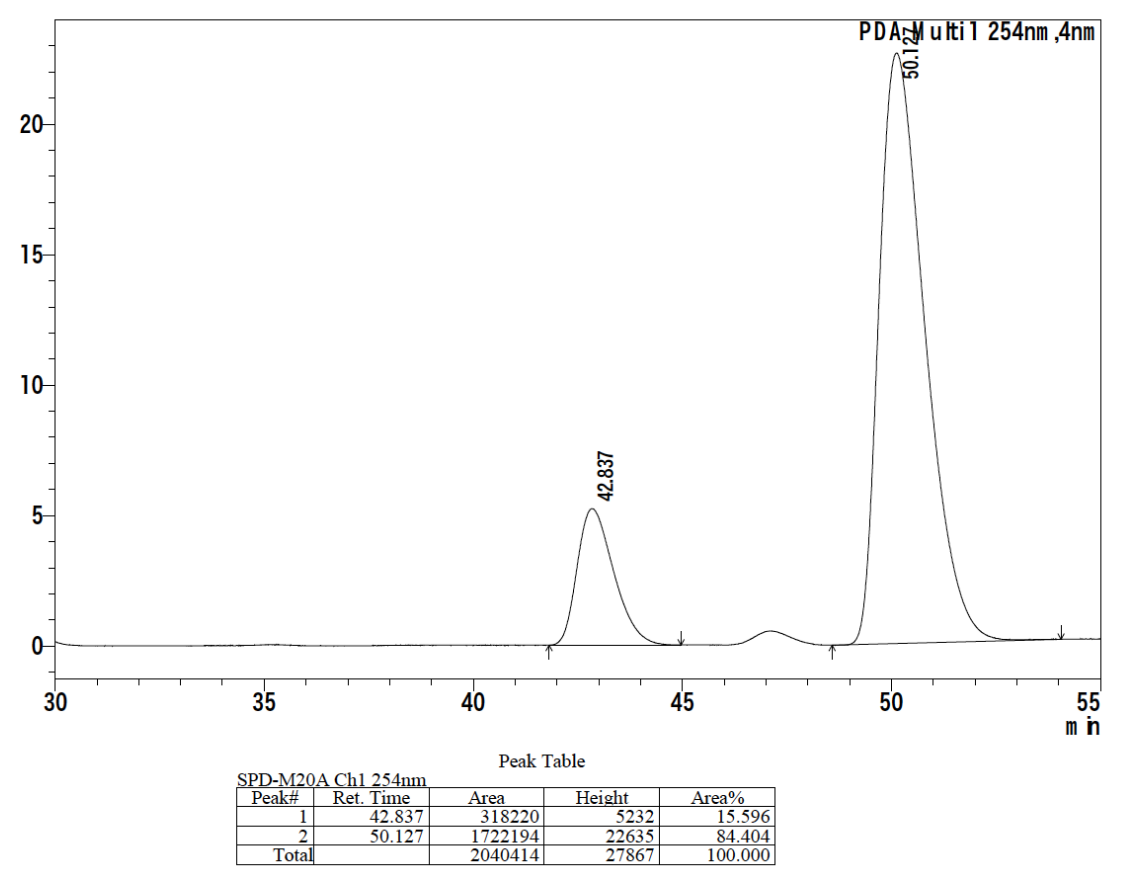




\section{HPLC Charts of 4c}<smiles>C#CCC(C)(O)c1ccc(Br)cc1</smiles>

Racemic 4c

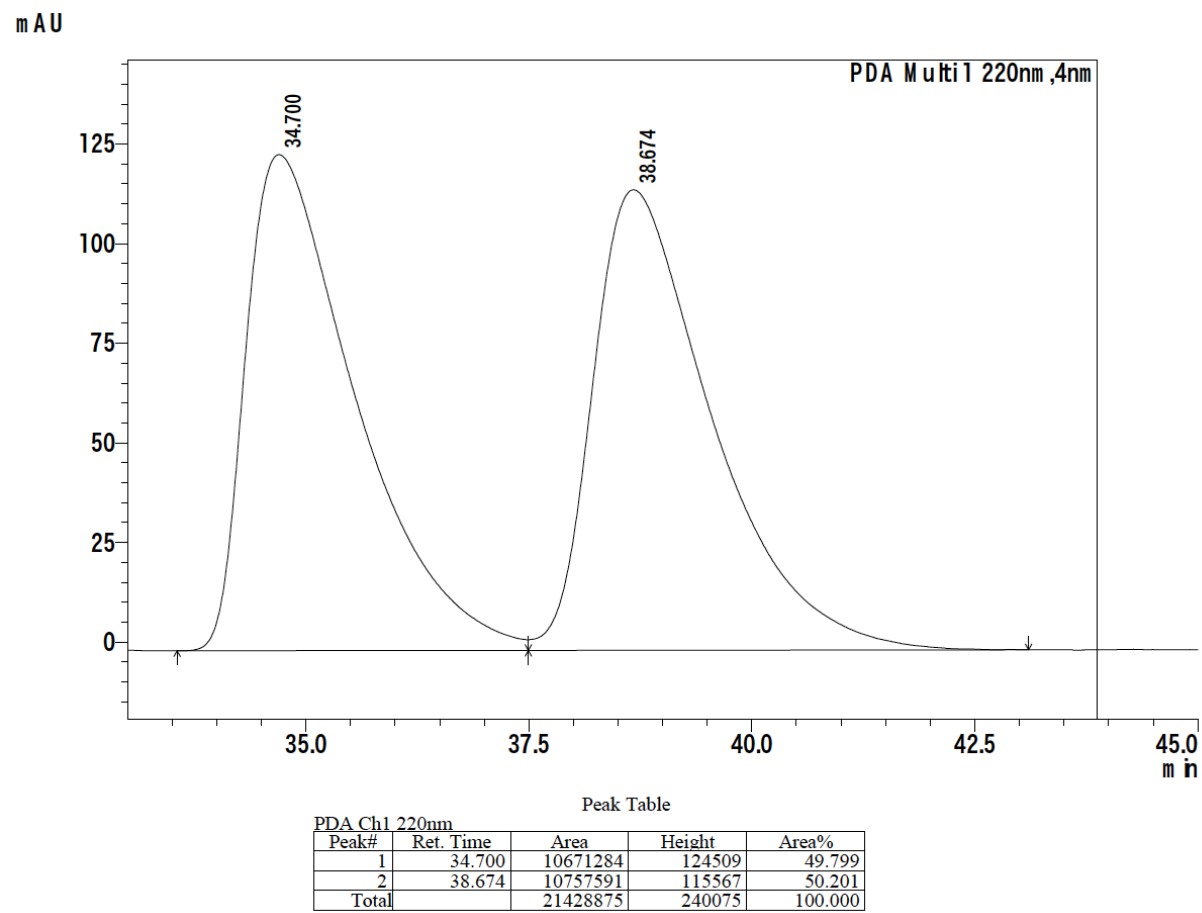

Optically active 4c

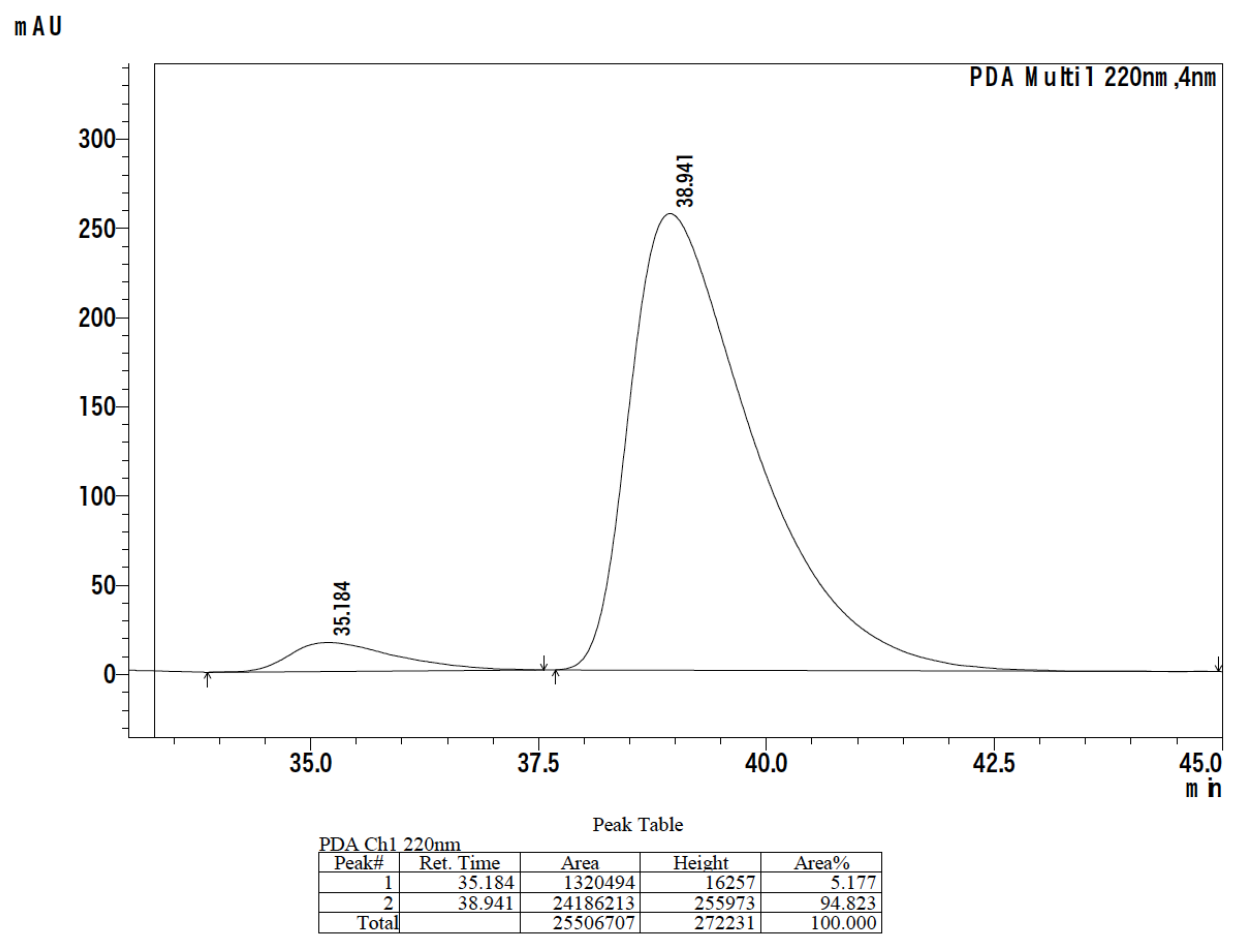

